\title{
IMAGING of \\ HAEMOPHILIC ARTHROPATHY
}

Wouter Foppen 


\section{Imaging of Haemophilic Arthropathy}

PhD thesis, Utrecht University, the Netherlands

Copyright (C) W. Foppen, 2016

All rights reserved. No part of this publication may be reproduced or transmitted in any form or by any means without the permission from the author. The copyright of articles that have been published or accepted for publication has been transferred to the respective journals.

ISBN/EAN: 978-90-393-6511-3

Design: N.P. Foppen

Lay-out: R. Sanders

Printer by: GVO drukkers \& vormgevers B.V. 


\section{IMAGING of HAEMOPHILIC ARTHROPATHY}

Beeldvorming van gewrichtsschade ten gevolge van hemofilie (met een samenvatting in het Nederlands)

\section{Proefschrift}

ter verkrijging van de graad van doctor aan de Universiteit Utrecht op gezag van de rector magnificus, prof. dr. G.J. van der Zwaan, ingevolge het besluit van het college van promoties in het openbaar te verdedigen op donderdag 14 april 2016 des ochtends te 10.30 uur

door

\section{Wouter Foppen}

geboren 24 januari 1988 te Harderwijk 
Promotor: $\quad$ Prof. dr. W.P.T.M. Mali

Copromotoren: Dr. K. Fischer

Dr. I.C. van der Schaaf 
The research in this thesis was financially supported by an unrestricted research grant from Baxalta B.V. The ultrasound scanner and training were funded by an unrestricted grant from Pfizer B.V.

Financial support for the publication of this thesis was generously provided by:

Baxalta B.V. | Bayer B.V. | Chipsoft B.V. | CSL Behring B.V. | Esaote B.V. | Novo Nordisk B.V. | Pfizer B.V. 


\section{Table of contents}

1 General introduction 9

\section{Part I. X-rays}

2 Scoring haemophilic arthropathy on X-rays: Improving inter- and intra-observer reliability and agreement with a consensus atlas

\section{Part II. Magnetic Resonance Imaging}

3 Haemophilic MRI score in healthy controls playing sports 41

4 Is joint effusion on MRI specific for haemophilia? 53

5 MRI changes predict joint bleeding and progression of arthropathy $\quad 67$ in haemophilia patients

\section{Part III. Point-of-care ultrasound}

6 Point-of-care ultrasound in haemophilic arthropathy: diagnostic accuracy compared to MRI

7 Value of point-of-care ultrasound in detecting early joint changes in children with haemophilia

\section{General discussion and Summary}

8 General discussion

9 Summary

Addenda

Nederlandse samenvatting (Dutch summary)

Review committee

Dankwoord (Acknowledgements)

Biography

List of publications 
CHAPTER 1

General introduction 
Haemophilia is a rare $\mathrm{X}$-linked recessive genetic disorder causing a deficiency or functional defect of coagulation factor VIII in haemophilia A or factor IX in haemophilia B. The prevalence is approximately 1 in 5000 males. In the absence of clotting factor replacement therapy, bleeding tendency is dependent on the residual clotting factor activity and patients are classified accordingly as: mild ( $>5 \%$ factor VIII or IX), moderate $(1-5 \%$ factor VIII or IX) or severe haemophilia ( $<1 \%$ factor VIII or IX) [1]. Severe haemophilia is characterized by spontaneous or minimal-trauma related bleeds, with 70$80 \%$ of bleeds occurring in the large joints (haemarthrosis) [2], especially in the elbows, knees and ankles [3-5]. Haemophilia patients treated with intermediate dose prophylactic clotting factor replacement therapy still have approximately two joint bleeds annually [6]. As repeated joint bleeding leads to joint destruction, arthropathy is a common comorbidity in haemophilia patients.

\section{Haemophilic arthropathy}

Multifactorial mechanisms of blood-induced joint damage have been identified. After a joint bleed, intra-articular blood directly induces chondrocyte apoptosis before being cleared by the synovial tissue. Haemosiderin is the breakdown product of intra-articular blood and accumulates in the synovial tissue [7]. Intra-articular blood, apoptotic chondrocytes, and haemosiderin, cause hypertrophy and hyper vascularisation of the synovial tissue resulting in an increased bleeding risk [8-10]. The blood-induced inflammatory processes in the synovial tissue and degenerative changes in cartilage influence each other and may occur in parallel and/or sequentially $[11,12]$.

\section{Treatment}

Coagulation factor replacement therapy for haemophilia A and B has been available since the late 1960s [13]. Especially regular replacement therapy in the absence of bleeding (prophylaxis) has proven to be very effective in preventing bleeding and arthropathy [14]. Early prophylaxis, preferably continued for life, is the recommended treatment for patients with severe haemophilia [2,15]. Unfortunately, high clotting factor consumption is associated with high costs. For adults with severe haemophilia, the mean annual costs of prophylaxis depend on the regimen used and vary between 180,000 US\$ (Dutch intermediate dose regimen) and 298,000 US\$ (Swedish high dose regimen) [16]. 


\section{Outcome assessment}

In order to improve patient outcome and improve the cost-effectiveness of therapy, optimising the use of costly coagulation factors is an on-going subject of study. Outcome assessment is used to evaluate the results of treatment in individual patients or between patient groups in (international) comparative studies.

\section{Patient reported outcomes}

Bleeding is the logical primary outcome to evaluate the effectiveness of prophylactic clotting factor replacement therapy [14]. Bleeding is however mostly patient-reported and symptoms of bleeding overlap with those caused by arthropathy [17]. Furthermore, definitions of bleeding vary among different reports [18]. Other patient-reported outcomes, such as quality of life or participation questionnaires, assess effects beyond the direct consequences of treatment [19].

\section{Imaging of haemophilic arthropathy}

In addition to patient reported outcome and physical examination, imaging of joints has always played an important role to assess outcome in haemophilia. Haemophilic arthropathy can be assessed using imaging techniques such as X-rays, Magnetic Resonance Imaging (MRI), and ultrasound (Figure 1).

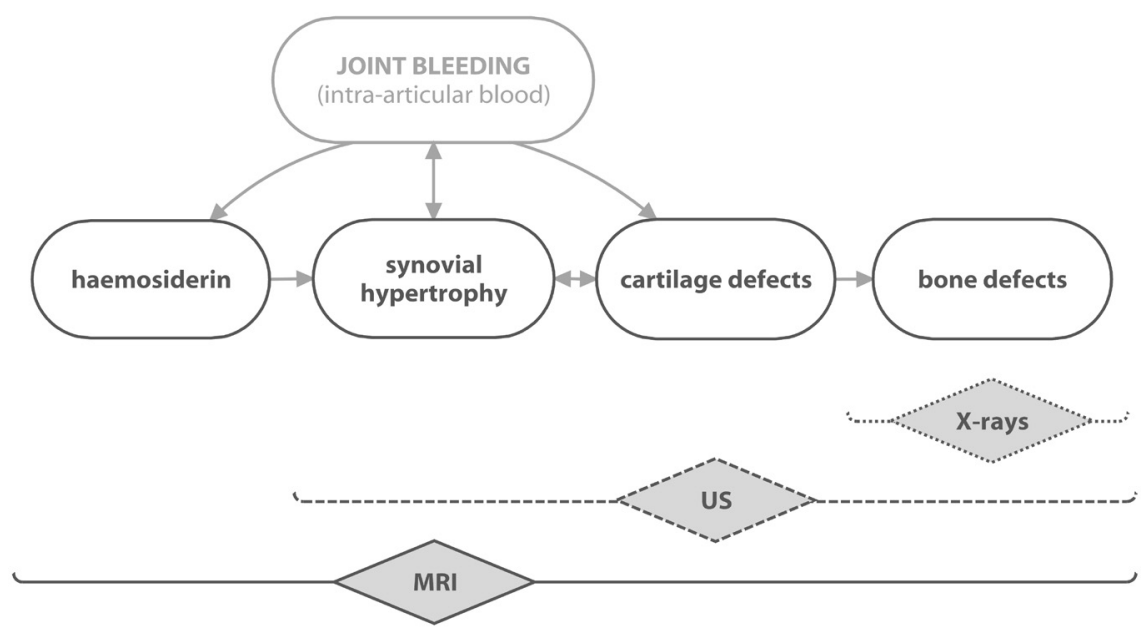

Figure 1. Imaging techniques to assess different aspects of haemophilic arthropathy 
As conventional X-ray imaging is inexpensive and universally available, it is a useful tool for regular follow-up and international comparisons of different clotting factor replacement strategies. Traditionally, joints are examined with standard X-rays and with haemophilic specific scores to assess late bony changes $[3,20]$. However, the first signs of haemophilic arthropathy occur in synovium and cartilage. MRI is a sensitive imaging modality to detect soft tissue changes as well as minor osteochondral changes [21]. Therefore, there is an increasing interest in the use of MRI to assess early, potentially reversible, joint changes in patients with normal findings on physical examination and X-rays. Compared to X-rays, MRI is less available, more time-consuming and more expensive. Therefore, MRI is not the first choice for routine joint assessment in the absence of major clinical complaints. Joint effusions, synovial hypertrophy, and cartilage defects (in some joint areas) can be assessed by ultrasound too and show strong correlations with MRI findings [22-25]. Various medical doctors perform ultrasound examinations themselves for specific indications, known as point-of-care ultrasound (POCUS) [26]. Recently, a straightforward POC-US protocol was developed for non-radiologists to allow direct evaluation of joint status in haemophilia [27].

\section{Outline of this thesis}

\section{Part I: X-rays}

The radiological Pettersson score is widely applied for classification of haemophilic arthropathy but its reproducibility was never evaluated. So, how can we interpret reported Pettersson scores? As it is unknown to what extent differences in scores are attributable to observer-variation, the aim of Chapter 2 was to assess and improve the inter- and intra-observer reliability and agreement of the Pettersson score using a consensus atlas.

\section{Part II: Magnetic Resonance Imaging}

With the increased interest in MRI assessment of joints in haemophilia, the questions arise regarding the clinical meaning of findings. Are MRI findings due to normal wear-and-tear or disease specific? Do MRI changes predict future bleeding and progression of arthropathy? In order to provide context for joint evaluation by MRI in young haemophilic patients, a cohort of young active healthy men was evaluated by MRI in Chapter 3. By comparison with healthy controls, the specificity of joint effusion in haemophilia patients was evaluated in Chapter 4. In order to facilitate clinical interpretation, the 
predictive value of MRI findings for five year joint bleeding and progression of arthropathy was assessed in Chapter $\mathbf{5}$.

\section{Part III: Point-of-care ultrasound}

Direct information on joint health may be useful to monitor treatment and to evaluate consequences of bleeding. May POC-US be useful for haemophilic arthropathy? The diagnostic accuracy of POC-US for haemophilic arthropathy was assessed by comparison with MRI in Chapter 6. Routine use of ultrasound is expected to be most relevant in patients with no or limited arthropathy. The value of POC-US for detection of early joint abnormalities was explored in children with haemophilia in Chapter 7.

\section{General Discussion and Summary}

Current knowledge and open issues regarding imaging of haemophilic arthropathy are discussed in a broader perspective in Chapter 8. The major findings in this thesis are summarized in Chapter 9. 


\section{References}

1 White GC, Rosendaal F, Aledort LM, et al. Definitions in hemophilia. Recommendation of the scientific subcommittee on factor VIII and factor IX of the scientific and standardization committee of the International Society on Thrombosis and Haemostasis. Thromb Haemost 2001; 85: 560.

2 Srivastava A, Brewer AK, Mauser-Bunschoten EP, et al. Guidelines for the management of hemophilia. Haemophilia 2013; 19: e1-47.

3 Arnold WD, Hilgartner MW. Hemophilic arthropathy. Current concepts of pathogenesis and management. J Bone Jt Surg Am 1977; 59: 287-305.

4 Aronstam A, Rainsford SG, Painter MJ. Patterns of bleeding in adolescents with severe haemophilia A. Br Med J 1979; 1: 469-70.

5 Ahlberg A. Haemophilia in Sweden. VII. Incidence, treatment and prophylaxis of arthropathy and other musculo-skeletal manifestations of haemophilia A and B. Acta Orthop Scand Suppl 1965 Suppl 77:3-132.

6 den Uijl I, Biesma D, Grobbee D, Fischer K. Turning severe into moderate haemophilia by prophylaxis: are we reaching our goal? Blood Transfus 2013; 11: 364-9.

7 van Vulpen LFD, Roosendaal G, van Asbeck BS, et al. The detrimental effects of iron on the joint: a comparison between haemochromatosis and haemophilia. J Clin Pathol 2015; 68: 592-600.

8 Roosendaal G, Vianen ME, Wenting MJ, et al. Iron deposits and catabolic properties of synovial tissue from patients with haemophilia. J Bone Joint Surg Br 1998; 80: 540-5.

9 Roosendaal G, TeKoppele JM, Vianen ME, et al. Blood-induced joint damage: A canine in vivo study. Arthritis Rheum 1999; 42: 1033-9.

10 Bhat V, Olmer M, Joshi S, et al. Vascular remodeling underlies rebleeding in hemophilic arthropathy. Am J Hematol 2015; 00: n/a - n/a.

11 Jansen NW, Roosendaal G, Lafeber FP. Understanding haemophilic arthropathy: an exploration of current open issues. Br J Haematol 2008; 143: 632-40.

12 Lafeber FPJG, Miossec P, Valentino L a. Physiopathology of haemophilic arthropathy. Haemophilia 2008; 14: 3-9.

13 Pool JG, Shannon AE. Production of high-potency concentrates of antihemophilic globulin in a closed-bag system. N Engl J Med 1965; 273: 1443-7.

14 Iorio A, Marchesini E, Marcucci M, Stobart K, Chan A. Clotting factor concentrates given to prevent bleeding and bleeding-related complications in people with hemophilia A or B (Review ). Cochrane Database Syst Rev 2011 1-46.

15 Berntorp E, Shapiro AD. Modern haemophilia care. Lancet 2012; 379: 1447-56.

16 Fischer K, Steen Carlsson K, Petrini P, et al. Intermediate-dose versus high-dose prophylaxis for severe hemophilia: comparing outcome and costs since the 1970s. Blood 2013; 122: 1129-36.

17 Timmer MA, Pisters MF, de Kleijn P, et al. Differentiating between signs of intra-articular joint bleeding and chronic arthropathy in haemophilia: a narrative review of the literature. Haemophilia 2015; 21: 289-96.

18 Chai-Adisaksopha C, Hillis C, Thabane L, Iorio A. A systematic review of definitions and reporting of bleeding outcome measures in haemophilia. Haemophilia 2015; 21: 731-5. 
19 Poonnoose P, Keshava S, Gibikote S, Feldman BM. Outcome assessment and limitations. Haemophilia 2012; 18 suppl 4: 125-30.

20 Pettersson H, Ahlberg A, Nilsson IM. A radiologic classification of hemophilic arthropathy. Clin Orthop Relat Res 1980; 149: 153-9.

21 Chan MW, Leckie A, Xavier F, et al. A systematic review of MR imaging as a tool for evaluating haemophilic arthropathy in children. Haemophilia 2013; 19: e324-34.

22 Zukotynski K, Jarrin J, Babyn PS, et al. Sonography for assessment of haemophilic arthropathy in children: a systematic protocol. Haemophilia 2007; 13: 293-304.

23 Querol F, Rodriguez-Merchan EC. The role of ultrasonography in the diagnosis of the musculoskeletal problems of haemophilia. Haemophilia 2012; 18: e215-26.

24 Aznar JA, Abad-Franch L, Perez-Alenda S, et al. Ultrasonography in the monitoring of management of haemarthrosis. Haemophilia 2011; 17: 826-8.

25 Di Minno MND, Iervolino S, Soscia E, et al. Magnetic resonance imaging and ultrasound evaluation of "healthy" joints in young subjects with severe haemophilia A. Haemophilia 2013; 19: e167-73.

26 Moore CL, Copel JA. Point-of-Care Ultrasonography. N Engl J Med 2011; 364: 749-57.

27 Martinoli C, Della Casa Alberighi O, di Minno G, et al. Development and definition of a simplified scanning procedure and scoring method for Haemophilia Early Arthropathy Detection with Ultrasound (HEAD-US). Thromb Haemost 2013; 109: 1170-9. 



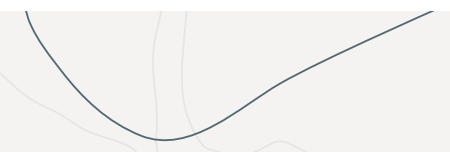

\section{Part I $\mathrm{X}$-rays}

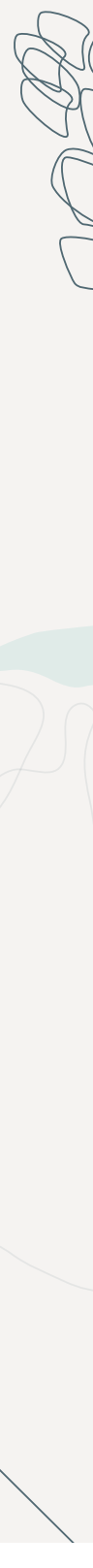


CHAPTER 2

\section{Scoring haemophilic} arthropathy on $\mathrm{X}$-rays:

improving inter- and intra-observer reliability and agreement using a consensus atlas 


\section{Abstract}

\section{Introduction}

The radiological Pettersson score (PS) is widely applied for classification of arthropathy to evaluate costly haemophilia treatment. This study aims to assess and improve inter- and intra-observer reliability and agreement of the PS.

\section{Methods}

Two series of X-rays (bilateral elbows, knees, and ankles) of 10 haemophilia patients (120 joints) with haemophilic arthropathy were scored by three observers according to the PS (maximum score 13/joint). Subsequently, (dis-)agreement in scoring was discussed until consensus. Example images were collected in an atlas. Thereafter, second series of 120 joints were scored using the atlas. One observer rescored the second series after three months. Reliability was assessed by intraclass correlation coefficients (ICC), agreement by limits of agreement (LoA).

\section{Results}

Median Pettersson score at joint level (PS joint $_{\text {of affected joints was } 6}$ (interquartile range 3-9). Using the consensus atlas, inter-observer reliability

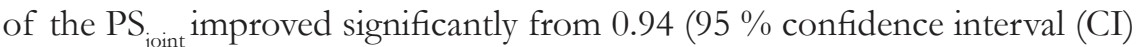
$0.91-0.96$ ) to 0.97 (CI 0.96-0.98). LoA improved from \pm 1.7 to \pm 1.1 for the $\mathrm{PS}_{\text {joint }}$. Therefore, true differences in arthropathy were differences in the $\mathrm{PS}_{\text {joint }}$ of $>2$ points. Intra-observer reliability of the $\mathrm{PS}_{\text {joint }}$ was 0.98 (CI 0.97-0.98), intra-observer LoA were \pm 0.9 points.

\section{Conclusions}

Reliability and agreement of the PS improved by using a consensus atlas. 


\section{Introduction}

Severe haemophilia is characterized by spontaneous or trauma-related joint bleeds. Recurrent joint bleeds eventually result in progressive arthropathy through metabolic and mechanical joint destruction [1]. The radiological Pettersson score (PS) was already designed in 1980 and is widely applied to classify the osteochondral changes of haemophilic arthropathy in elbows, knees, and ankles [2]. It is used to evaluate the effects of different treatment strategies, especially in international studies [2-4]. The PS is an additive score consisting of eight items (Table 1).

Table 1. Classification of haemophilic arthropathy according to the Pettersson score [2]

\begin{tabular}{lll}
\hline \multirow{2}{*}{ Osteoporosis } & Absent & 0 \\
& Present & 1 \\
\hline \multirow{2}{*}{ Enlargement of epiphysis } & Absent & 0 \\
& Present & 1 \\
\hline \multirow{2}{*}{ Irregularity of subchondral surface } & Absent & 0 \\
& Partially involved & 1 \\
\hline \multirow{2}{*}{ Narrowing of joint space } & Totally involved & 2 \\
\hline \multirow{3}{*}{ Subchondral cysts formation } & Absent & 0 \\
& Joint space $>1 \mathrm{~mm}$ & 1 \\
\hline \multirow{2}{*}{ Erosion of joint margins } & Joint space $<1 \mathrm{~mm}$ & 2 \\
\hline \multirow{2}{*}{ Gross incongruence of articulating bone ends } & Absent & 0 \\
& 1 cyst & 1 \\
& $>1$ cyst & 2 \\
\hline \multirow{2}{*}{ Joint deformity (angulation and/or displacement) } & Absent & 0 \\
& Present & 1 \\
\hline
\end{tabular}

Some of these items leave room for subjective interpretation. This is not a problem if all joints are scored by a single observer. However, a reproducible PS is especially important in comparative international studies involving multiple observers focusing on small differences. These comparative international studies are performed to justify the high costs of clotting factor replacement therapy. Although magnetic resonance imaging (MRI) is the most sensitive 
imaging modality for evaluation of arthropathy, it is too costly and too time consuming for use in routine follow up. In contrast, routine plain X-rays of the main joints at 5-year intervals are recommended in routine follow-up of patients with severe haemophilia [5]. As conventional radiography is cheap and universally available, it is a useful tool for international comparisons of different clotting factor replacement strategies.

The reproducibility of a scoring method involves the reliability and agreement. Reliability is a measure to define how well patients can be differentiated with the tool of interest. Agreement is the extent in which scores by different observers are identical $[6,7]$. Although the PS is available for over 30 years, only three studies have assessed its reproducibility [8-10]. Formal assessment of the limits of agreement, however, was not performed. Yet, these limits of agreement are important for the clinical interpretation of the results. Without knowledge on the reproducibility of the PS, it is not clear whether small differences in scores represent real differences in arthropathy, or are attributable to inter-observer variation.

The original paper about the PS describes the genesis of the score, including the radiological changes, which are included in the score. However, definitions or examples of the included radiological items were not provided. Improved definition of PS items is expected to improve reliability and agreement [8, 9]. An atlas with reference images of different stages of haemophilic arthropathy of different joints and corresponding PS could potentially be a helpful tool. The purpose of this research was to develop a consensus atlas for the PS and to evaluate the impact of this atlas on the inter-observer and intra-observer reliability and agreement.

\section{Materials and methods}

This study was conducted according to the Guidelines for Reporting Reliability and Agreement Studies (GRRAS) [7]. Inter-observer and intraobserver reproducibility of the Pettersson score at joint level $\left(\mathrm{PS}_{\text {joint }}\right)$ and the Pettersson score at patient level $\left(\mathrm{PS}_{\text {patient }}\right)$ were assessed in this study. The study was approved by the institutional ethical review board, and informed consent was waived.

\section{Pettersson score}

The PS is based on typical findings of haemophilic arthropathy on posterioranterior and lateral X-rays, including osteoporosis, enlargement of epiphysis, 
irregularity of subchondral surface, narrowing of joint space, subchondral cysts, erosions at joint margins, incongruence between joint surfaces, and the angulation and/or displacement of articulating bone ends (Table 1). The maximum $\mathrm{PS}_{\text {joint }}$ is 13 points. The PS ${ }_{\text {patient }}$ represents the sum of the six joints (elbows, knees, and ankles) with a maximum score of 78 points.

\section{Sample size}

Although determining sample size for studies on reliability and agreement is not straightforward [7], Shoukri and colleagues provided approximate sample sizes depending on the expected reliability values and the number of observers. For two observers and expected intraclass correlation coefficients larger than 0.80 , inclusion of about 50 joints are needed [11], as about $50 \%$ of joints of haemophilia patients are affected on X-rays [12]. A sample size of $\geq 100$ joints in the present study would suffice.

\section{Patients and observers}

Two series (mean time between series: 5.6 years) of X-rays (bilateral elbows, knees, and ankles) of 20 patients representing the full range of radiological variation were scored in this study. These patients were included based on previous PS by an experienced observer [13]. The current study involved three observers with different levels of experience in using the PS: one radiologist with experience using the PS (scoring $>450$ joints previously [3]), one radiologist without experience using the PS, and a medical doctor $(\mathrm{PhD}$ candidate on imaging of haemophilic arthropathy) without experience using the PS.

The reproducibility prior to the consensus atlas was evaluated by assessing the first series of X-rays (ten patients; 120 joints). These X-rays were scored independently by three observers. Ankylosis, arthroplasty, or arthrodesis

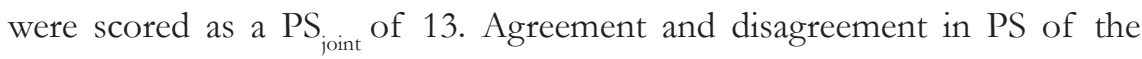
first series of $\mathrm{X}$-rays were discussed in three consensus meetings. Common disagreements were discussed until consensus was reached. Example images with descriptions of the PS items were collected into a consensus atlas. Subsequently, the effect of the consensus atlas was evaluated: the same three observers independently scored the second series of X-rays (ten different patients; 120 different joints) with use of the developed consensus atlas. To assess the intra-observer reliability and agreement while using the consensus atlas, the second series of X-rays were rescored after 3 months by the medical doctor without experience using the PS, blinded for the first results. 


\section{Analysis}

The Mann-Whitney $\mathrm{U}$ test and Chi-squared test (two-sided) were used to compare distributions of patient characteristics between the first series of $\mathrm{X}$-rays scored prior to the consensus atlas and the second series of $\mathrm{X}$-rays scored with the consensus atlas. Generalized estimating equations (GEE) were performed to test whether PS were statistically different between the series of X-rays and to correct for repeated measurements and clustering of joints within patients. Due to variation of data distributions, three models were used: a binary model for comparison of percentages of abnormal scores, a gamma with log link model for comparison of medians across all scores, and a linear model for comparison of medians of abnormal scores only. The $p$ values represent the adjusted analyses of the difference between the two series of $\mathrm{X}$-rays. $P$ values less than 0.05 were considered statistically significant.

Inter-observer and intra-observer reliability were assessed by two-way random intraclass correlation coefficientagreement (ICC) with $95 \%$ confidence intervals (CI) for both the PS joint $_{\text {and the PS }}$ patient . The ICC is a measure to evaluate the relation between the variance in subjects and the variance in scores caused by the different observers. An ICC of 0.00 means a poor reliability among observers; an ICC of 1.00 means a perfect reliability. The reliability of the PS was considered significantly different in case the CI regarding the PS prior to the consensus atlas was not overlapping the ICC of the PS with use of the consensus atlas.

Agreement was assessed by a graphical method for multiple observers in a single plot according to Jones and colleagues [14], which is comparable to a Bland-Altman plot for two observers $[14,15]$. The difference between each observer and the overall mean of all observers was calculated including the limits of agreement from the mean (LoA) ( \pm 2 standard deviations). The difference from the mean for each observer was subsequently plotted. The minimal detectable difference in PS can be interpreted as the change in PS beyond the maximal variance in scores caused by different observers. For the used method to assess agreement, the range between the higher and lower LoA (twice the LoA) could be interpreted as the minimal detectable difference. Analyses were performed in SPSS (IBM SPSS Statistics version 20, Armank, NY).

All six imaged joints of patients were included the analyses and results regarding reliability and agreement. Though most patients with haemophilia 
only have only several joint with frequent bleeds and subsequent arthropathy, about $50 \%$ of joints were affected [12]. Healthy joints are easy to score. For that reason, a subgroup analysis of abnormal PS was performed to study the reliability and agreement of these affected joints only. An abnormal PS was defined as a PS $>0$ according to the mean of the three observers.

\section{Results}

X-rays of 240 joints in total from 20 patients were assessed. Joints with the whole range of haemophilic arthropathy were scored $\left(\mathrm{PS}_{\text {joint }} 0-13\right.$ points). Median age of patients was 35.4 years (inter quartile ranges (IQR) 28.6-46.1). Based on serum coagulation factor VIII / IX levels, fourteen patients had severe haemophilia A $\left(\right.$ FVIII $\left.<1 \mathrm{IU} \mathrm{dL}^{-1}\right)$, three had moderate haemophilia A (FVIII 2-5 IU dL ${ }^{-1}$ ), two had severe haemophilia B (FIX $<1 \mathrm{IU} \mathrm{dL}^{-1}$ ), and one patient had moderate haemophilia B (FIX 2-5 IU dL ${ }^{-1}$ ). Scored joints included three ankles with previous joint surgery (arthrodesis) (Table 2).

Table 2. Patient and joint characteristics of the series of $\mathrm{X}$-rays scored without the consensus atlas and the series of X-rays scored with the consensus atlas

\begin{tabular}{lccc} 
& $\begin{array}{c}\text { Series without } \\
\text { consensus atlas } \\
(10 \text { patients, } 120 \text { joints })\end{array}$ & $\begin{array}{c}\text { Series with } \\
\text { consensus atlas } \\
(10 \text { patients, } 120 \text { joints })\end{array}$ & $P$-value \\
\hline Age (years) & $35.8(27.7-47.5)$ & $33.7(28.8-43.4)$ & 0.97 \\
Type of haemophilia (A / B) & $90 \% / 10 \%$ & $80 \% / 20 \%$ & 1.00 \\
Severity (moderate / severe) ${ }^{\text {a }}$ & $20 \% / 80 \%$ & $20 \% / 80 \%$ & 1.00 \\
Treatment (on demand / prophylactic) & $40 \% / 60 \%$ & $10 \% / 90 \%$ & 0.30 \\
Joints with surgery & $1.7 \%$ & $3.3 \%$ & 1.00 \\
& & & \\
Pettersson score at joint level & $64 \%$ & $49 \%$ & $0.28^{\mathrm{b}}$ \\
Percentage abnormal scores & $3(0-7)$ & $0(0-4)$ & $0.45^{\mathrm{b}}$ \\
Median of all scores & $6(3-9)$ & $4(2-9)$ & $0.67^{\mathrm{b}}$ \\
Median of abnormal scores only & & & \\
Pettersson score at patient level & $90 \%$ & $85 \%$ & $0.73^{\mathrm{b}}$ \\
Percentage abnormal scores & $25(10-36)$ & $9(2-23)$ & $0.28^{\mathrm{b}}$ \\
Median of all scores & $26(19-37)$ & $12(7-24)$ & $0.24^{\mathrm{b}}$ \\
\hline Median of abnormal scores only & & & \\
\hline
\end{tabular}

Values are percentages and medians (inter quartile ranges) regarding the average score of the three observers

a Severity of disease according to true serum levels of coagulation factors VIII in haemophilia A or IX in haemophilia B

b Calculated using regression analyses adjusted for repeated measurements and clustering of joints within patients 
A consensus atlas with example images and descriptions of the items (e.g., Figure 1) was established and is now available as Electronic Supplementary Material.

Overall, median PS joint $_{\text {was }} 1$ (IQR 0-7) and the median PS ${ }_{\text {patient }}$ was 19 (IQR 7-28). A PS joint $_{\text {above } 0 \text { was observed in 136/240 (57\%) joints and a PS }}$ above 0 in 35/40 (88\%) patients. The first series of X-rays (scored prior to the consensus atlas) included more joints with a positive PS (64\% with
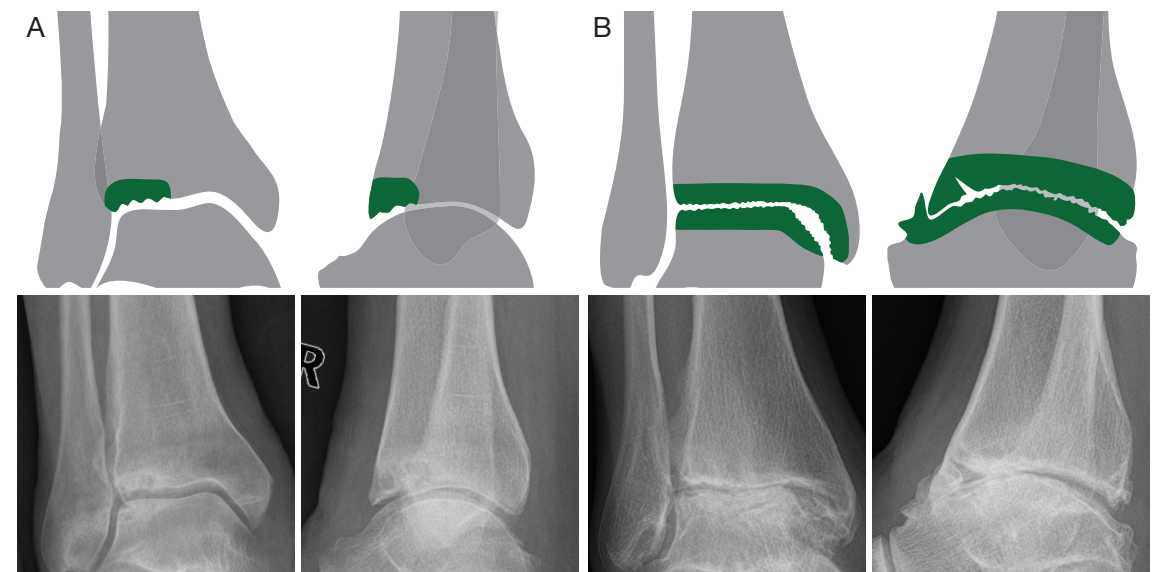

Figure 1. Example images from the consensus atlas of 'irregularity of subchondral surface' in the ankle. A) Partially involved, minor subchondral cysts present as well. B) Totally involved, other significant joint alterations present as well

$\left.\mathrm{PS}_{\text {joint }}>0\right)$ compared to the second series of $\mathrm{X}$-rays, which was scored with the consensus atlas (49\% with $\left.\mathrm{PS}_{\text {joint }}>0\right)$. A subgroup analysis of the abnormal Pettersson scores only is provided at the end of the results section. Patient characteristics and the range of scores were comparable among both series of X-rays.

\section{Reliability}

Inter-observer reliability (reflected by the ICC) of the PS ${ }_{\text {joint }}$ significantly improved from 0.94 (CI 0.91-0.96) to 0.97 (CI 0.96-0.98) with use of the consensus atlas (Table 3). For the PS ${ }_{\text {patient, }}$, the reliability significantly improved from 0.94 (CI 0.86-0.98) to 0.99 (CI 0.97-1.00). The intra-observer reliability, which was only assessed with use of the consensus atlas, was excellent; 0.98 (CI 0.97-0.98) for the PS joint $_{\text {and } 0.99 \text { (CI 0.98-1.00) for the PS }}$ patient. 
Table 3. Inter-observer and intra-observer reliability without the consensus atlas, and with use of the consensus atlas regarding the Pettersson score at joint level and the Pettersson score at patient level

\begin{tabular}{|c|c|c|c|c|}
\hline & \multicolumn{2}{|c|}{ Pettersson score at joint level } & \multicolumn{2}{|c|}{ Pettersson score at patient level } \\
\hline & Without atlas & With atlas & Without atlas & With atlas \\
\hline \multicolumn{5}{|c|}{ Results regarding all Pettersson scores } \\
\hline Inter-observer & $0.94(0.91-0.96)$ & $0.97(0.96-0.98) *$ & $0.94(0.86-0.98)$ & $0.99(0.97-1.00)^{*}$ \\
\hline Intra-observer & - & $0.98(0.97-0.98)$ & - & $0.99(0.98-1.00)$ \\
\hline \multicolumn{5}{|c|}{ Subgroup analysis of abnormal Pettersson scores only } \\
\hline Inter-observer & $0.88(0.81-0.92)$ & $0.94(0.91-0.96)^{*}$ & $0.92(0.80-0.97)$ & $0.99(0.97-0.99)^{*}$ \\
\hline Intra-observer & - & $0.96(0.93-0.97)$ & - & $0.99(0.97-1.00)$ \\
\hline
\end{tabular}

Values represent the intraclass correlation coefficients ( $95 \%$ confidence intervals).

* Significant improvement of the reliability with use of the consensus atlas

\section{Agreement}

Before establishment of the consensus atlas, the experienced radiologist deviated the least from the mean score of the three observers (Figs. 2 and 3). Agreement among the observers as expressed by inter-observer LoA before the consensus atlas was \pm 1.7 points for the PS $_{\text {joint }}$ (maximum score 13 ). The observers deviated less from the mean score after using the consensus atlas: the LoA for the $\mathrm{PS}_{\text {joint }}$ improved to \pm 1.1 points with use of the consensus atlas (Table 4). Regarding the intra-observer agreement after the consensus atlas, the LoA were \pm 0.9 points for PS $_{\text {joint }}$. Therefore, the minimal detectable difference for the $\mathrm{PS}_{\text {joint }}$ could be interpreted as $>2$ points (rounded) after the consensus atlas. Thus, differences in $\mathrm{PS}_{\text {ioint }}>2$ points are attributable to true differences in arthropathy in case the $\mathrm{PS}_{\text {joint }}$ is scored with use of the consensus atlas.

For the PS ${ }_{\text {patient }}$ (maximum score 78), the inter-observer LoA improved from \pm 6.5 points prior to the consensus atlas to \pm 3.2 points with use of the consensus atlas (Table 4). The intra-observer LoA after the consensus atlas were \pm 2.4 points. In other words, the minimal detectable difference of the PS $_{\text {patient }}$ scored by different observers halved with use of the consensus atlas from $>13$ points (rounded) to $>6$ points (rounded). 

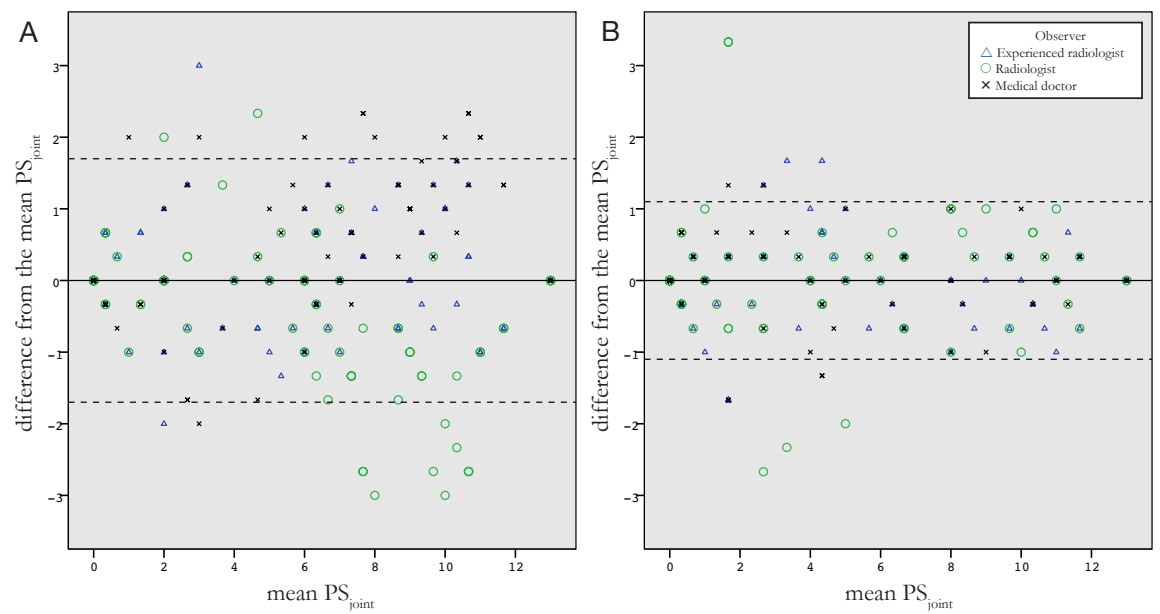

Figure 2. Plot of inter-observer agreement regarding the Pettersson score at joint level (PS ioint $)$. A) Without consensus atlas. B) With consensus atlas. Horizontal dotted lines indicate the limits of agreement from the mean (LoA) of the three observers. Some symbols are superimposed. With use of the consensus atlas, the LoA improved from \pm 1.7 to \pm 1.1 at joint level
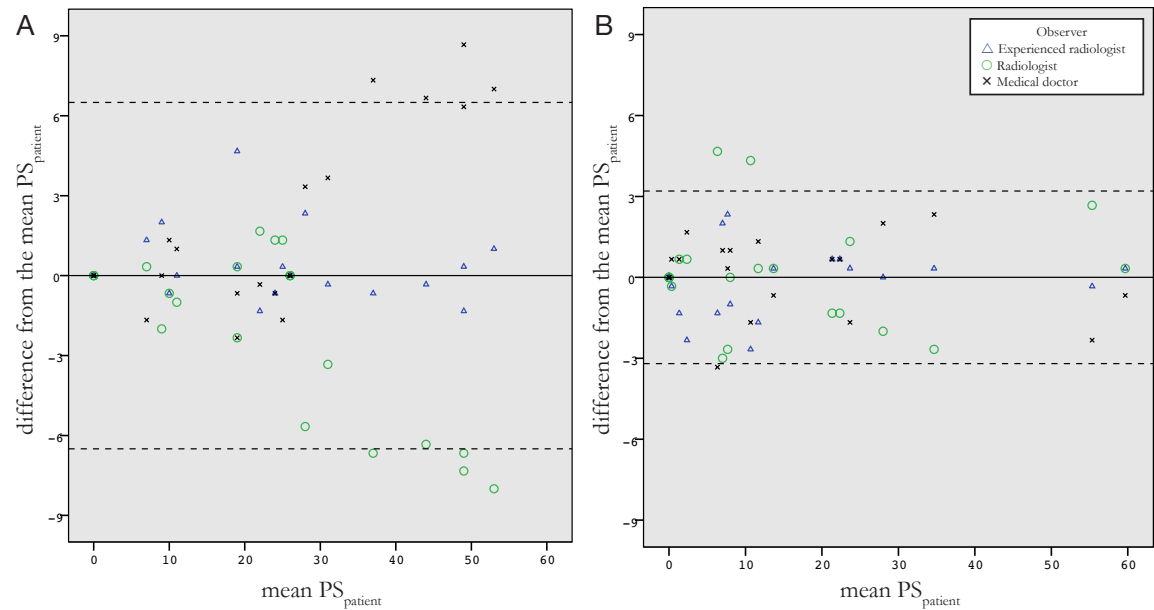

Figure 3. Plot of inter-observer agreement regarding the Pettersson score at patient level (PS ${ }_{\text {patient }}$ ). A) Without consensus atlas. B) With consensus atlas. Horizontal dotted lines indicate the limits of agreement from the mean (LoA) of the three observers. Some symbols are superimposed. With use of the consensus atlas the LoA for the total PS improved from \pm 6.5 to \pm 3.2 
Table 4. Inter-observer and intra-observer agreement without the consensus atlas, and with use of the consensus atlas regarding the Pettersson score at joint level and the Pettersson score at patient level

\begin{tabular}{|c|c|c|c|c|}
\hline & \multicolumn{2}{|c|}{ Pettersson score at joint level } & \multicolumn{2}{|c|}{ Pettersson score at patient level } \\
\hline & Without atlas & With atlas & Without atlas & With atlas \\
\hline \multicolumn{5}{|c|}{ Results regarding all Pettersson scores } \\
\hline Inter-observer & $\pm 1.7(>3)$ & $\pm 1.1(>2)$ & $\pm 6.5(>13)$ & $\pm 3.2(>6)$ \\
\hline Intra-observer & - & $\pm 0.9(>2)$ & - & $\pm 2.4(>5)$ \\
\hline \multicolumn{5}{|c|}{ Subgroup analysis of abnormal Pettersson scores only } \\
\hline Inter-observer & $\pm 2.1(>4)$ & $\pm 1.5(>3)$ & $\pm 6.9(>14)$ & $\pm 3.4(>7)$ \\
\hline Intra-observer & - & $\pm 1.3(>3)$ & - & $\pm 2.6(>5)$ \\
\hline
\end{tabular}

Values represent the limits of agreement from the mean (minimal detectable difference)

\section{Subgroup analysis of abnormal Pettersson scores only}

In total, $57 \%$ of scored joints were affected by arthropathy. Median PS of all affected joints was 6 (IQR 3-9) and the median of all PS ${ }_{\text {patient }}$ was 22 (IQR 9-31). Additional analyses of abnormal PS (i.e., with at least one joint abnormality, PS $>0$ ) were performed in order to test the effect of the atlas on reliability and agreement of the $\mathrm{PS}_{\text {joint }}$ and $\mathrm{PS}_{\text {patient }}$ in a comparable population with the whole range of haemophilic arthropathy.

Also in this subgroup analysis, the consensus atlas improved reliability and agreement of the PS. The reliability of the PS ${ }_{\text {joint }}$ significantly improved from 0.88 (CI 0.81-0.92) prior to the consensus atlas to 0.94 (CI 0.91-0.96) with

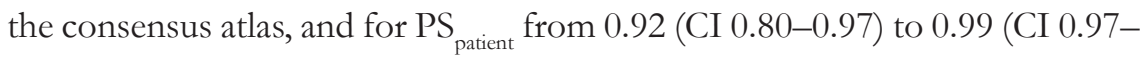
0.99 ) with use of the consensus atlas (Table 3). LoA improved from \pm 2.1 to \pm 1.5 points for $\mathrm{PS}_{\text {joint }}$, and from \pm 6.9 to \pm 3.4 points for the $\mathrm{PS}_{\text {patient }}$ (Table 4 ).

\section{Discussion}

This reproducibility study is the first to assess both reliability and agreement of the Pettersson score (PS) and the effects of using a consensus atlas. Interobserver reliability of the PS ${ }_{\text {joint }}$ and PS ${ }_{\text {patient }}$ significantly improved with use of the developed consensus atlas. The minimal detectable difference reduced with use of the consensus atlas as a result of improved agreement among observers.

Results of improved reliability and agreement should be interpreted with care. The reproducibility of the PS with use of the consensus atlas might be overestimated by two causes. First, the second series of joints scored 
had less affected joints overall (Table 2). As healthy joints are easy to score, the reliability and agreement of the PS is likely better in series of X-rays with many healthy joints compared to series of X-rays with many affected joints. As expected, the subgroup analyses of only affected joints (PS $>0$ ) showed a lower reliability and agreement of the PS compared to the analyses of all joints including the healthy joints (PS $\geq 0$ ). Nonetheless, the subgroup analyses of only affected joints still showed that the consensus atlas resulted in an improved reliability and agreement of the PS. Since the reproducibility of the PS is influenced by the severity of arthropathy, the external validity of the results regarding reliability and agreement of all scores, including healthy joints, is limited to haemophilia patients with a comparable severity of haemophilic arthropathy. However, the results of the subgroup analyses are externally valid in affected joints representing the whole range of arthropathy.

Second, the improved reliability and agreement with use of the consensus atlas might be caused by a learning effect. In the series of X-rays scored before the consensus atlas, the experienced radiologist deviated the least from the mean score. While scoring the second series of X-rays with use of the consensus atlas, the unexperienced radiologist and medical doctor already scored 120 joints. This might have improved the reliability and agreement to a certain extent, in addition to the consensus atlas. An external validation study could be performed to assess whether the reliability and agreement of the PS is comparable when the consensus atlas is used by different observers.

The established consensus atlas is available as Electronic Supplementary Material to illustrate our interpretations of abnormalities. As this consensus atlas is subjective, observers in other treatment centres or countries might interpret abnormalities differently. If so, the consensus atlas could be discussed internationally to establish a global consensus atlas in order to offer a widely supported tool for a reproducible PS.

\section{Relation to other studies}

The three available studies regarding the reproducibility of the PS date from 1989, 2008, and 2011 [8-10]. Unfortunately, these studies cannot be compared with our results. The study by Erlemann et al. focused on the agreement of single items of the PS [8]. Results on agreement by LoA were not provided for the $\mathrm{PS}_{\text {joint }}$ or the PS ${ }_{\text {patient. }}$ Yet, the LoA would have been useful since they provide clinical relevant information on the minimal detectable difference. Differences in PS larger than the minimal detectable difference, 
thus exceeding the possible inter-observer or intra-observer differences, are caused by true differences in arthropathy as scored by the PS.

The more recent studies by Silva et al. and Takedani et al. focused on the reliability of the PS and other radiographic scores for haemophilic arthropathy $[9,10]$. However, their results cannot be compared sufficiently with our results, as different statistical methods were used. Results regarding the reliability of the PS $_{\text {ioint }}$ according to Silva et al. and Takedani et al. were poor according to the kappa statistic: 0.06 and 0.12 respectively $[9,10]$. Since the kappa statistic is designed to evaluate the reliability of nominal data (e.g., present/absent or failed/passed), only identical scores are taken into account. The PS can be interpreted as ordinal or interval data. For that reason, we assessed the reliability of the PS ${ }_{\text {joint }}$ and PS ${ }_{\text {patient }}$ according to the ICC for interval data that incorporates the magnitude of differences between observers. This is likely one of the reasons that the results of reliability in our study are better compared to Silva et al. and Takedani et al.

In addition, healthy joints of haemophilia patients were included in the current study. As described above, including healthy joints results in a better reliability and agreement since these joints are easy to score. However, patient and joint characteristics in the previous studies are not provided. Therefore, it is not possible to formally assess whether inclusion of healthy joints of haemophilia patients caused the observed differences in reliability between the previous studies and our study.

The reliability of the PS in our study was already good before development of the consensus atlas (ICC > 0.90) and even improved significantly with its use. Therefore, these findings suggest that PS is a reliable tool to score the severity of haemophilic arthropathy on X-rays. Agreement among observers was limited before the consensus atlas. With use of the consensus atlas, the observers were more consistent. For interpretation of our results with use of the consensus atlas, true differences in arthropathy according to the PS were differences of $>2$ points for PS $_{\text {ioint }}$ (maximum score 13) and changes of $>6$ points for $\mathrm{PS}_{\text {patient }}$ (maximum score 78).

Further research could focus on validation of the consensus atlas. In such a validation study, different observers (who were not involved in establishing the consensus atlas) will score series of X-rays to specifically evaluate the effect of the consensus atlas on the reproducibility of the PS. 


\section{Conclusion}

Reliability and agreement of the PS could be improved with the use of a consensus atlas. Use of a consensus atlas to score haemophilic arthropathy according to the PS is recommended in order to improve reliability and to lower the minimal detectable difference.

\section{Electronic supplementary material}

Below are the links to the electronic supplementary material.
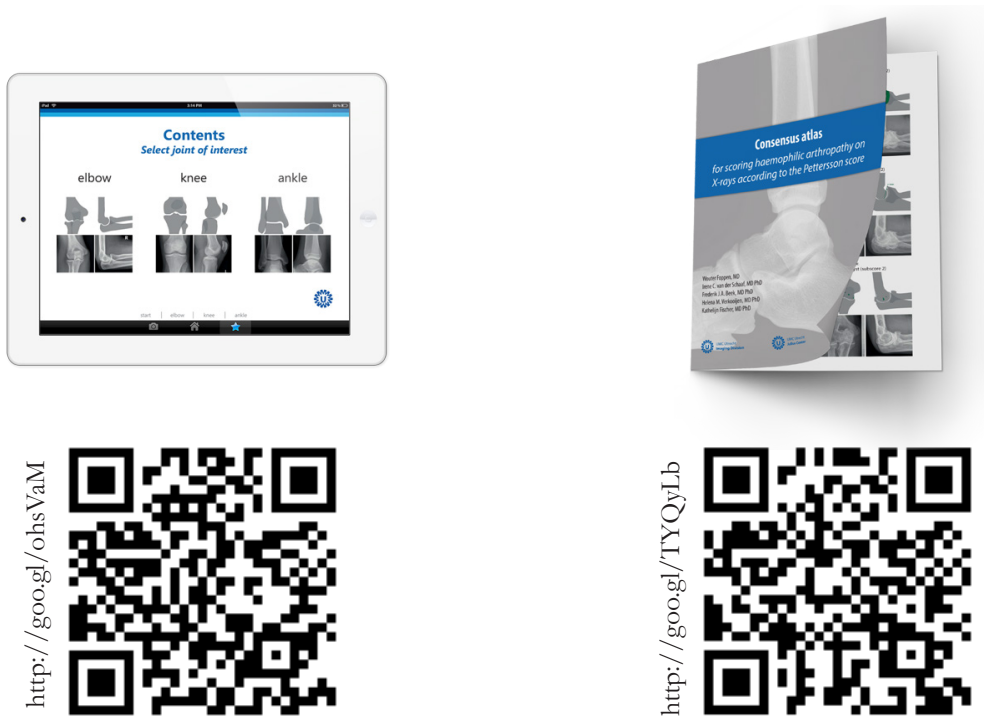


\section{References}

1 Jansen NW, Roosendaal G, Lafeber FP. Understanding haemophilic arthropathy: an exploration of current open issues. Br J Haematol 2008; 143: 632-40.

2 Pettersson H, Ahlberg A, Nilsson IM. A radiologic classification of hemophilic arthropathy. Clin Orthop Relat Res 1980; 149: 153-9.

3 Van den Berg HM, Fischer K, Mauser-Bunschoten EP, et al. Long-term outcome of individualized prophylactic treatment of children with severe haemophilia. Br J Haematol 2001; 112: 561-5.

4 Fischer K, Astermark J, van der Bom JG, et al. Prophylactic treatment for severe haemophilia: comparison of an intermediate-dose to a high-dose regimen. Haemophilia 2002; 8: 753-60.

5 De Moerloose P, Fischer K, Lambert T, et al. Recommendations for assessment, monitoring and follow-up of patients with haemophilia. Haemophilia 2012; 18: 319-25.

6 De Vet HCW, Terwee CB, Knol DL, Bouter LM. When to use agreement versus reliability measures. J Clin Epidemiol 2006; 59: 1033-9.

7 Kottner J, Audigé L, Brorson S, et al. Guidelines for Reporting Reliability and Agreement Studies (GRRAS) were proposed. J Clin Epidemiol 2011; 64: 96-106.

8 Erlemann R, Rosenthal H, Walthers EM, Almeida P, Calleja R. Reproducibility of the pettersson scoring system. An interobserver study. Acta Radiol 1989; 30: 147-51.

9 Silva M, Luck Jr. J V, Quon D, et al. Inter- and intra-observer reliability of radiographic scores commonly used for the evaluation of haemophilic arthropathy. Haemophilia 2008; 14: 504-12.

10 Takedani H, Fujii T, Kobayashi Y, Haga N, Tatsunami S. Inter-observer reliability of three different radiographic scores for adult haemophilia. Haemophilia 2011; 17: 134-8.

11 Shoukri M, Asyali M, Donner a. Sample size requirements for the design of reliability study: review and new results. Stat Methods Med Res 2004 251-71.

12 Aledort LM, Haschmeyer RH, Pettersson H. A longitudinal study of orthopaedic outcomes for severe factor-VIII-deficient haemophiliacs. The Orthopaedic Outcome Study Group. J Intern Med 1994; 236: 391-9.

13 Fischer K, van der Bom JG, Mauser-Bunschoten EP, et al. Changes in treatment strategies for severe haemophilia over the last 3 decades: effects on clotting factor consumption and arthropathy. Haemophilia 2001; 7: 446-52.

14 Jones M, Dobson A, O’Brian S. A graphical method for assessing agreement with the mean between multiple observers using continuous measures. Int J Epidemiol 2011; 40: 1308-13.

15 Bland JM, Altman DG. Statistical methods for assessing agreement between two methods of clinical measurement. Lancet 1986; 1: 307-10. 



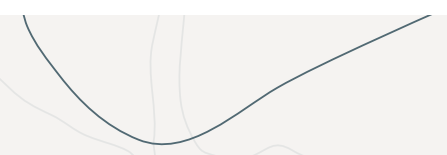

\section{Part II}

\section{Magnetic Resonance Imaging}

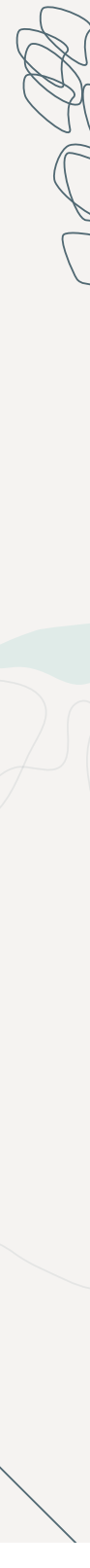




\section{CHAPTER 3 \\ Haemophilic MRI score in healthy controls playing sports}

W. Foppen ${ }^{1}$, D. Sluiter ${ }^{2}$, T.D. Witkamp ${ }^{1}$, W.P.T.M. Mali ${ }^{1}$ K. Fischer ${ }^{3,4}$ 1 Department of Radiology; 2 Department of Rehabilitation, Nursing Science and Sports; 3 Julius Center for Health Sciences and Primary Care; and 4 Van Creveldkliniek, Department of Hematology, University Medical Center Utrecht, 


\section{Abstract}

\section{Introduction}

Magnetic resonance imaging (MRI) is the most sensitive imaging modality to assess joint lesions, but the clinical relevance of subtle joint changes in haemophilic patients playing sports is unknown. A haemophilia specific MRI score is available, but was never evaluated in physically active healthy controls. It is not known if unexpected MRI changes in young active haemophilic patients are due to sports participation. The aim of this study was to evaluate knees and ankles in a cohort of young active healthy men using a haemophilia specific MRI score to provide context for joint evaluation by MRI in young haemophilic patients.

\section{Methods}

Three Tesla MRI of knees and ankles were performed in 30 healthy men aged 18-26 years, regularly active in sports. MR images were scored by a single independent radiologist, using the International Prophylaxis Study Group additive MRI score. One physiotherapist assessed clinical function using the Haemophilia joint health scores (HJHS). History of complaints or injuries affecting knees and/or ankles, very intensive sports and current sports activities were documented.

\section{Results}

Median age was 24.3 years (range 19.0-26.4) and median number of sports activities per week was 3 (range 1-4). Six joints (five knees, one ankle) had a history of a sports-related injury. The median HJHS per joint was 0 out of 20 (range $0-1$ ). All joints had a MRI score of 0 .

\section{Conclusions}

These results suggest that regular sports participation or very low HJHS scores are not associated with haemophilia specific MRI changes in knees and ankles. 


\section{Introduction}

Haemophilia is an X-linked recessive genetic disorder characterized by spontaneous or trauma-related bleeds, especially in the large joints. Recurrent joint bleeds eventually lead to synovial hypertrophy, cartilage and bone damage by mechanical and metabolic joint destruction [1,2]. For followup and staging of haemophilic arthropathy two scoring systems based on radiography have been available since the early 1980s. The additive Pettersson score is based on eight different findings [3], and the progressive score of the Arnold-Hilgartner scale is based on the worst finding in five categories [4]. With the intensification of treatment, the requirements for monitoring are changing. Radiography is suitable for the detection of bony changes associated with advanced arthropathy, but soft tissue changes of early disease remain undetected. Magnetic resonance imaging (MRI) is the most sensitive imaging modality available to assess joint lesions. The International Prophylaxis Study Group (IPGS) developed a MRI scoring system for analysis of early joint diseases $[5,6]$. This standardized MRI scoring system demonstrated excellent reliability and shows superior performance in discrimination of early osteoarticular changes compared to radiography [7].

Interpretation of radiological findings is not always straightforward in haemophilic patients: they may represent anatomic variants, be caused by wear and tear [8], non-haemophilic disease, traumatic injuries, or they may be haemophilia related. A clinical trail by Manco-Johnson et al. [9] showed a positive MRI score in boys without a history of joint bleeds suggesting that the changes were caused by occult bleeds. Den Uijl et al. [10] performed a prospective study to assess haemophilia specific MRI changes in young adult haemophilia patients without clinical or radiological evidence of arthropathy. Knees and ankles of 26 young adults with severe or moderate haemophilia were scanned using three Tesla MRI equipment. Only mild MRI changes were observed. However, 3 out of 18 patients who were active in sports showed unexpected MRI changes in joints without a history of bleeds [10]. It is not known if those unexpected MRI changes were due to sports participation or occult bleeding. Interpretation is difficult, as haemophilia specific MRI has not been performed in physically active adults and the effects of regular sports participation on the outcome of the MRI score are not known.

The aim of our study was to evaluate knee and ankle joints in a cohort of young active healthy men using a haemophilia specific MRI score to provide context for joint evaluation by MRI in young haemophilic patients. 


\section{Materials and methods}

Healthy young males aged 18-26 years, performing regular sports were invited through university message boards. Exclusion criteria were a contra-indication for MRI or a history of joint injury within 8 weeks prior the examination. Knees and ankles of 30 subjects were scanned by MRI, and clinical function was assessed according to the HJHS by a single physiotherapist on the same day [11]. Type and frequency of current sports activities, including a history of frequent sports participation ( $\geq 4$ times per week) in the past, were recorded by the physiotherapist. All subjects were examined if they had a history of any complaints or injuries possibly affecting knees and/or ankles.

The research protocol was approved by the Institutional Review Board of UMC Utrecht, the Netherlands. All subjects signed informed consent.

\section{Imaging}

Joints were imaged separately using MR equipment (Philips, type Achieva 3T TX; Koninklijke Philips Electronics NV, the Netherlands) with field strength of 3 Tesla using a 16-Channel Knee Coil. A scanning protocol substantially corresponding to the protocol described by Den Uijl et al. was used to for an improved detection of haemosiderin deposits and synovial hypertrophy and to allow a total scanning time of $<60$ min for both knees and ankles and [10, 12]. The total time in the MRI room was $<70 \mathrm{~min}$.

Scanning protocol consisted of 3D Water only Selection (WATS) [Gradient echo (GRE); repetition time $(\mathrm{TR})=20 \mathrm{~ms}$, echo time $(\mathrm{TE})=4.4 \mathrm{~ms}$, flip angle (FA) $35^{\circ}$ ] with section thickness $2 \mathrm{~mm}$ sagittal for knees and coronal for ankles, with reconstruction in three planes for detection of synovial hypertrophy and haemosiderin deposits. Proton Density Weighted (PDW) Spectral Adiabatic Inversion Recovery (SPAIR) [SE, TR $=3000 \mathrm{~ms}$, TE = $30 \mathrm{~ms}$, inversion time $(\mathrm{TI})=100 \mathrm{~ms}$, sagittal and transversal with section thickness $3.5 \mathrm{~mm}$ for knees and coronal with section thickness $2.5 \mathrm{~mm}$ for ankles for detection of cartilage loss and subchondral cysts. T1-weighted [Spin echo $(\mathrm{SE}) ; \mathrm{TR}=500 \mathrm{~ms}, \mathrm{TE}=20 \mathrm{~ms}$ ] coronal with section thickness $3.5 \mathrm{~mm}$ for knees and sagittal with section thickness $2.5 \mathrm{~mm}$ for ankles were added to provide optimal anatomical overview of both joints.

\section{Scoring}

All MRI images were scored by a single independent radiologist with 25 years musculoskeletal experience, blinded for clinical function, according to the 
additive MRI score (max. 20 points/joint) of the International Prophylaxis Study Group (IPSG) (Table 1) [5]. This additive score summarizes findings of synovial hypertrophy, haemosiderin, changes of subchondral bone or joint margins and cartilage loss. To ascertain that joints were normal on clinical examination one physiotherapist assessed clinical function using the HJHS version 2.1 (max. 20 points/joint, max. HJHS total score for knees and ankles is 84$)$ [11].

Table 1. Twenty-step additive magnetic resonance imaging score of the International Prophylaxis Study Group [5]

Additive scale (A)

Synovial hypertrophy
Small
Moderate
Large
Haemosiderin
Changes of subchondral bone or joint margins
Any surface erosion
Any surface erosion in at least two bones
Half or more of the articular surface eroded in at least one bone
Half or more of the articular surface eroded in at least two bones
At least one subchondral cyst
More than one subcondral cyst
Subchondral cysts in at least two bones
Multiple subchondral cysts in each of at least two bones
Cartilage loss
Any loss of joint cartilage height
Any loss of joint cartilage height in at least two bones
Any loss of joint cartilage height involving more than one third of the
joint surface in at least one bone
Any loss of joint cartilage height involving more than one third of the
joint surface in at least two bones
Full-thickness loss of joint cartilage in at least some area in at least one bone
Full-thickness loss of joint cartilage in at least some area in at least two bones
Full-thickness loss of joint cartilage involves at least one third of the joint surface
in at least two bones

Add numbers $(\max$ value $=20)$

(A) - 


\section{Power calculation}

Using a Fisher exact test with a one-sided $\alpha=0.05$ and $\beta=0.20$, scanning 30 control subjects would suffice to detect a difference of $36 \%$ or higher in prevalence of osteochondral changes on MRI between haemophilic patients: $70 \%[10]$ and young active healthy men: 34\% [13].

\section{Analysis}

Medians, inter quartile ranges (IQR) and percentages were calculated for the patient characteristics. For each joint, the MRI score was compared with the HJHS to assess agreement. Agreement was defined when both scores were positive or when both scores were negative.

\section{Results}

In total, 120 joints (60 knees and 60 ankles) of 30 healthy young active adults were scanned using MRI. Subject characteristics are show in Table 2. Median age was 24.3 years (range 19.0-26.4) and median number of sports activities per week was 3 (range 1-4). A history of more than three sports activities a week (4-8 times a week) was reported by 11 subjects. In total, six joints (five knees, one ankles) had a history of an injury more than 8 weeks prior to the examination. Single subjects reported different sports activities; the three most frequently reported sports were running (60\%), fitness $(33 \%)$ and tennis $(17 \%)$.

Table 2. Subject characteristics

\begin{tabular}{lc}
\hline & $\mathrm{n}=30$ subjects, 120 joints \\
\hline Age (years) & $24.3[23.2-25.4]$ \\
Active in sports (times a week) & $3[2-3]$ \\
$4-8$ sports activities a week in the past & $5(16.7 \%)$ \\
Ankle injury & $1(2 \%)$ \\
Knee injury & $5(8 \%)$ \\
Reported sport activities (number of subjects) & Running 18; Fitness 10; Tennis 5; Cycling 4; Football 4; \\
& Rugby 3; Squash 3; Golf 2; Kitesurfing 2; Rowing 1; \\
& Swimming 1; Jiu Jitsu 1; Survival races 1 \\
\hline
\end{tabular}

Values are median (inter quartile ranges) or $\mathrm{n}(\%)$. Different sport activities were reported by single subjects. 22 out of the 30 subjects performed at least one high impact sport (underlined). 
Physical examination scores and MRI scores at joint level are shown in Table 3. Clinical function of most joints was very good, 105 joints (88\%) scored zero on the HJHS. Positive scores were due to crepitus on motion in the knee or loss of flexion of the ankle. The 15 positive joint scores were each caused by one finding, the median HJHS joint total was zero (range 0-1) out of 20 points per joint. Of the 120 joints scanned by MRI, none of the joints showed synovial hypertrophy, haemosiderin, changes of subchondral bone or joint margins and no cartilage loss of the femur or tibia. Therefore, all subject had a MRI score of zero. However, some abnormalities were observed which were not included in the haemophilia specific MRI score. One knee $(1.7 \%)$ showed irregular retro-patellar cartilage, three knees $(5 \%)$ showed a meniscal tear, and bone marrow oedema was seen in four knees (6.7\%) and one ankle (1.7\%). Two of the five subjects with those findings reported frequent participation in sports in the past with 4-8 sports activities per week longer than a year ago. The HJHS of the joints with abnormalities were 0 , except for one knee with irregular retro-patellar cartilage (HJHS $=1$, due to mild crepitus on motion).

Table 3. Agreement of additive magnetic resonance imaging (MRI) score and haemophilia joint health scores (HJHS) in knee and ankle joints of young and active male subjects on joint level

\begin{tabular}{llll}
\hline Joint & MRI score & HJHS & $\mathrm{n}$ \\
\hline Knee & 0 & 0 & $52(87 \%)$ \\
& 0 & $>0$ & $8(13 \%)$ \\
Ankle & 0 & 0 & $53(88 \%)$ \\
& 0 & $>0$ & $7(12 \%)$ \\
\hline
\end{tabular}

One subject with a meniscal tear in both knees reported to have an injury in both knees: a strain of the anterior cruciate ligament in one knee and a meniscal tear due to hyperextension in the other knee. The HJHS for both knees was 0 . The correlation of MRI score with the clinical function could not be calculated as all MRI scores were 0 .

\section{Discussion}

This study is the first to evaluate the association of haemophilia specific MRI changes and sports participation in a cohort of young active healthy men. In this control group, MRI did not show any haemophilia specific joint changes 
according to the IPGS additive score shown in Table 1, even in those with a history of a joint injury. Clinical function as assessed by the HJHS was optimal (score 0) in $88 \%$ of joints.

The present study included a group of patients of adequate size and used validated tools like the HJHS $[11,14]$ and the additive MRI score of the IPGS $[5,7]$. The choice of control subjects was limited to young physically active adults to provide a control group for our MRI study in haemophilia patients. In this MRI pilot study of 26 patients aged 13-26 years Den Uijl detected changes in cartilage in joints without a history of bleeding, but with a history of frequent sports activities [10]. To provide an adequate control group we choose men active in sports. Most subjects reported 1-3 sport activities per week, three controls reported four sports activities per week. The physical activity levels in the control subjects was in accordance with the male general population in the Netherlands: for subjects aged 15-25 years 38\% is active $1-4 \mathrm{~h}$ per week and $28 \%$ more than $5 \mathrm{~h}$ per week [15].

Although all MRI scores were $0,12 \%$ of the knees and $2 \%$ of the knees showed an abnormal finding not included in the additive MRI. Those findings indicate a certain amount of stress of the examined joints. These results suggest that normal sports participation will not affect the haemophilia specific MRI score, i.e. no synovial hypertrophy, haemosiderin, changes of subchondral bone or joint margins and cartilage loss should be expected due to sports activities in young haemophilic patients.

Our findings are in accordance with the few data available. One study evaluated abnormalities in knees in teenage soccer players (mean age 15 years) and in the age-related control group using a 0.35 T open-field MRI unit [13]. The soccer players trained 3-3.5 h per day for 5 days a week compared to a maximum of $1 \mathrm{~h}$ a week in the control group. In the group of soccer players 18 out of 24 knees $(64.3 \%)$ revealed one or more abnormalities, most frequently bone marrow oedema or infrapatellar fat pad oedema. In the control group 9 out of 28 knees $(32.1 \%)$ revealed abnormalities. However, items of the haemophilia specific MRI score [5] were not seen or scored. Especially abnormalities of weight bearing cartilage were not seen in any of the subjects.

In our study, we found one knee with irregular retro-patellar cartilage. However, retro-patellar cartilage is not mentioned specifically in the additive MRI score [5]. The additive MRI score includes 'Any loss of cartilage' and therefore may include scoring of retro-patellar cartilage changes. Because we 
did not see other changes we did not score this finding. As this is a matter of interpretation others could score the finding of irregular retro-patellar cartilage with one point: 'Any loss of cartilage'. If so, 1.7\% (CI 0.04-8.90\%) of knees would score positively in our study.

As the current study was performed in physically active young men aged 19-26 years, it does not provide information on normal MRI scores in older subjects. Signs of wear and tear usually include loss of cartilage height in weight bearing cartilage surfaces [8]. It is therefore likely that these will result in positive haemophilia specific scores with advancing age. This is corroborated by Stahl et al. [16] showing cartilage defects in 4 out of 10 marathon runners (mean age $31 \pm 5$ years, 5 training per week) and in 3 out of 12 controls (37 \pm 11 years, maximum 2 sports activities per week). Cartilage defects included retro-patellar findings in 2 out of 4 marathon runners and in 1 out of 3 controls. These findings suggest that retro-patellar cartilage defects are quite common in non-haemophilic subjects active in sports. Our study suggests that the points included in the additive MRI score [5] are haemophilia specific in younger patients. Excluding retro-patellar cartilage abnormalities may contribute to an even more haemophilia specific MRI assessment method.

The aim of this study was to help interpret the finding of cartilage lesions in haemophilic patients without a history of joint bleeds, but performing frequent sport activities [10]. With our results we cannot explain the positive scores due to cartilage lesions of weight bearing surfaces in haemophilic patients without reported joint bleeds $[9,10]$. It is very unlikely those lesions were caused by sports participation or injuries. This confirms that the MRI score used is very specific for haemophilic arthropathy in younger patients. Perhaps these cartilage changes were caused by occult bleeds [9], but as no haemosiderin deposits were observed, these must have occurred a long time ago. The origin of these cartilage changes may very well be multifactorial, i.e. a combination of injury, occult bleeding and joint loading as was observed in dog studies [17].

\section{Conclusion}

The results of our study suggest that knees and ankles of young healthy adults will show no haemophilia specific abnormalities on MRI. Regular sports participation or very low HJHS scores are not associated with haemophilia specific MRI changes in knees and ankles. 


\section{References}

1. Roosendaal G, Lafeber FP. Blood-induced joint damage in hemophilia. Semin Thromb Hemost 2003; 29: $37-42$

2. Jansen NW, Roosendaal G, Lafeber FP. Understanding haemophilic arthropathy: an exploration of current open issues. Br J Haematol 2008; 143: 632-40.

3. Pettersson H, Ahlberg A, Nilsson IM. A radiologic classification of hemophilic arthropathy. Clin Orthop Relat Res 1980; 149: 153-9.

4. Arnold WD, Hilgartner MW. Hemophilic arthropathy. Current concepts of pathogenesis and management. J Bone Joint Surg Am 1977; 59: 287-305.

5. Lundin B, Babyn P, Doria AS, Kilcoyne R, Ljung R, Miller S, et al. Compatible scales for progressive and additive MRI assessments of haemophilic arthropathy. Haemophilia 2005; 11: 109-15.

6. Feldman BM, Funk S, Lundin B, Doria AS, Ljung R, Blanchette V. Musculoskeletal measurement tools from the International Prophylaxis Study Group (IPSG). Haemophilia 2008; 14 Suppl 3: 162-9.

7. Doria AS, Lundin B, Kilcoyne RF, Babyn PS, Miller S, Nuss R, et al. Reliability of progressive and additive MRI scoring systems for evaluation of haemophilic arthropathy in children: expert MRI Working Group of the International Prophylaxis Study Group. Haemophilia 2005; 11: 245-53.

8. Donell ST. "Osteoarthritis" on imaging may be normal wear and tear. BMJ 2012; 345: e5594.

9. Manco-Johnson MJ, Abshire TC, Shapiro AD, Riske B, Hacker MR, Kilcoyne R, et al. Prophylaxis versus episodic treatment to prevent joint disease in boys with severe hemophilia. N Engl J Med 2007; 357: 535-44.

10. Den Uijl IE, De Schepper AM, Camerlinck M, Grobbee DE, Fischer K. Magnetic resonance imaging in teenagers and young adults with limited haemophilic arthropathy: baseline results from a prospective study. Haemophilia 2011; 17: 926-30.

11. Hilliard P, Funk S, Zourikian N, Bergstrom BM, Bradley CS, McLimont M, et al. Hemophilia joint health score reliability study. Haemophilia 2006; 12: 518-25.

12. Den Uijl IE, De Schepper AM, Grobbee DE, Fischer K. Evaluating therapy in hemophilia: development of an MRI protocol. Journal of Thrombosis and Haemostasis 2009; 7s2, PP-WE-534: 2.

13. Soder RB, Simoes JD, Soder JB, Baldisserotto M. MRI of the knee joint in asymptomatic adolescent soccer players: a controlled study. AJR Am J Roentgenol 2011; 196: W61-5.

14. Feldman BM, Funk SM, Bergstrom BM, Zourikian N, Hilliard P, van der Net J, et al. Validation of a new pediatric joint scoring system from the International Hemophilia Prophylaxis Study Group: validity of the hemophilia joint health score. Arthritis Care Res (Hoboken) 2011; 63: 223-30.

15. CBS. Vrije tijd; sport, hobby, cultuur, recreatie, vakantie. 2012 [cited 2012 11/13]; Available from: http://statline.cbs.nl/StatWeb/publication/?DM=SLNL\&PA=60029NED\&D1=0-2,6,19,56$63 \& \mathrm{D} 2=0-1,5 \& \mathrm{D} 3=\mathrm{a} \& H \mathrm{DR}=\mathrm{T} \& \mathrm{STB}=\mathrm{G} 1, \mathrm{G} 2 \& \mathrm{VW}=\mathrm{T}$.

16. Stahl R, Luke A, Ma CB, Krug R, Steinbach L, Majumdar S, et al. Prevalence of pathologic findings in asymptomatic knees of marathon runners before and after a competition in comparison with physically active subjects-a 3.0 T magnetic resonance imaging study. Skeletal Radiol 2008; 37: 627-38.

17. Hooiveld MJ, Roosendaal G, Jacobs KM, Vianen ME, van den Berg HM, Bijlsma JW, et al. Initiation of degenerative joint damage by experimental bleeding combined with loading of the joint: a possible mechanism of hemophilic arthropathy. Arthritis Rheum 2004; 50: 2024-31. 


\section{CHAPTER 4 \\ Is joint effusion on MRI specific for haemophilia?}

Published in Haemophilia (2014)

W. Foppen ${ }^{1}$, I.C. van der Schaaf ${ }^{1}$, T. D. Witkamp ${ }^{1}$, K. Fischer ${ }^{2,3}$

${ }^{1}$ Department of Radiology; ${ }^{2}$ Julius Center for Health Sciences and Primary Care; and ${ }^{3}$ Van Creveldkliniek, Department of Hematology, University Medical Center Utrecht, Utrecht, The Netherlands. 


\section{Abstract}

\section{Introduction}

Magnetic resonance imaging (MRI) scores for haemophilic arthropathy are useful for evaluation of early and moderate arthropathy. The most recent additive International Prophylaxis Study Group (IPSG) MRI scale for haemophilic arthropathy includes joint effusion. However, it is unknown whether joint effusion is haemophilia specific. Correct interpretation of joint effusion is needed for outcome assessment of prophylactic therapies in haemophilia care. The aim of this study was to compare joint effusion on MRI between young adults with haemophilia and healthy controls.

\section{Methods}

MRI's of both knees and ankles of 26 haemophilic patients (104 joints) and 30 healthy active men (120 joints) were assessed. Scans in both groups were performed in 2009/2010 and 2012 respectively. Joint effusion was measured and scored according to the MRI atlas referred by the IPSG MRI scale for haemophilic arthropathy.

\section{Results}

Median age of haemophilic patients and healthy controls was 21 and 24 years respectively. In haemophilic patients $23 \%$ of knees and $22 \%$ of ankles showed joint effusion. Healthy controls had significantly more positive scores for knee effusion $(67 \%, P<0.01)$ and a comparable scores for effusion in the ankle $(17 \%)$.

\section{Conclusions}

Joint effusion according to criteria of the IPSG MRI scale was observed significantly more often in knees of healthy controls, while findings in ankles were similar. These data suggest that joint effusion in knees and ankles is not haemophilia specific. Inclusion of joint effusion in the MRI scale is expected to reduce its specificity for haemophilic arthropathy. 


\section{Introduction}

The deficiency or functional defect of coagulation factors in haemophilia results in an increased bleeding tendency. Approximately $70-80 \%$ of bleeds in haemophilic patients occur in the large joints, especially the elbows, knees and ankles [1-3]. The Arnold-Hilgartner scale [2] and the Pettersson score [4] are available to quantify late osteochondral changes based on radiography. Magnetic Resonance Imaging (MRI) scores are the most sensitive assessment tool for haemophilic arthropathy. In 2005 the progressive Denver MRI score [5, 6] and the additive European MRI score [7] were combined in a comprehensive scoring scheme by the MRI subgroup of the international Prophylaxis Study Group (IPSG) [8]. This score was recently adapted to provide a single additive scale including joint effusion as additional item to the previous additive MRI score $[8,9]$. The MRI score showed good inter-rater reliability (intraclass correlation coefficient 0.88) [9-11].

The sensitivity of MRI for haemophilic arthropathy allows follow-up and evaluation of different treatment regimens in relatively young patients for scientific research [7, 8, 12]. Recently, knees and ankles of 30 healthy controls playing sports were evaluated using the 2005 version of the additive MRI score [8] to provide context for joint evaluation in young adults with haemophilia [13]. None of the joints had a positive MRI score according to the IPSG score and therefore the MRI score seemed to be very specific for haemophilic arthropathy in younger patients. However, the latest version of the MRI score takes joint effusion into account [9]. To study the specificity of this item, series of MRI's in haemophilic patients and healthy controls were assessed for joint effusion and compared.

The aim of this study was to provide context for interpretation of joint effusion in haemophilic patients by comparing joint effusion in haemophilic patients and healthy controls.

\section{Materials and methods}

Two series of MRI's of knees and ankles were assessed for the presence of joint effusion and compared. The first series concerned a cohort of all moderate and severe haemophilic patients from the University Medical Center Utrecht, the Netherlands (Van Creveldkliniek) with mild arthropathy (maximum 1 joint with a Pettersson score above 3). Out of 61 eligible patients, 26 patients (aged 12-29 years) volunteered in the study and were 
scanned in 2009/2010 [14]. The second series concerned healthy young men, aged 18-26 years and regularly active in sports. These subjects were invited through university message boards as a control group. The exclusion criterion for controls was a joint injury within 8 weeks before the examination. Knees and ankles of 30 controls were scanned in 2012 [13]. The Institutional Review Board of the UMC Utrecht, the Netherlands, approved the research protocol. Informed consent was signed by all subjects.

Self-designed questionnaires concerning type and frequency of sports performed in an average week in May had been administered previously for other studies in the haemophilia patients and at the day of MRI examination in the control group. Sports with impact on the lower extremity (impact sports) were defined as football, tennis, squash, running, field hockey, basketball, volleyball and rugby.

\section{Imaging}

Joints were imaged separately using the same three Tesla MR equipment (Philips, type Achieva 3T TX; Koninklijke Philips Electronics NV, the Netherlands) in both cohorts. A 16-Channel Knee Coil was used in the control group. The imaging protocol for the knee consisted of $2 \mathrm{~mm}$ sagittal 3D Water only Selection (WATS), a $3.5 \mathrm{~mm}$ sagittal and transversal Proton Density Weighted (PDW) Spectral Adiabatic Inversion Recovery (SPAIR) and a $3.5 \mathrm{~mm}$ coronal T1-weighted Spin echo (SE). For the ankle the imaging protocol consisted of $2 \mathrm{~mm}$ coronal 3D WATS, a $2.5 \mathrm{~mm}$ coronal PDW SPAIR and a $2.5 \mathrm{~mm}$ sagittal T1-weighted SE.

\section{Scoring}

In both cohorts, MR images were scored previously for the presence of synovial hypertrophy, haemosiderin, and osteochondral changes [13, 14]. For the current study, all MR images were reassessed for the presence of joint effusion by one of the authors (WF). Joint effusion was measured and scored according to the MRI atlas [15] referred by the most recent additive IPSG MRI scale for haemophilic arthropathy [9]. Measurements were performed by drawing a region of interest (ROI) around the effusion on a single slice on the sagittal image with the largest area of fluid intensity. Sagittal PDW SPAIR images were used for scoring effusion in knees. For ankles, effusion was scored on sagittal T1-weighted images linked with the coronal PDW SPAIR images for correct interpretation of joint effusion. 
For the knees, the measured effusion surface was categorized according to the MRI atlas into moderate (score 2: 1-2 $\mathrm{cm}^{2}$ ) and large effusion (score 3: $>2$ $\mathrm{cm}^{2}$ ). Small effusion (score 1) is defined as 'an area of fluid intensity is present but less than $1 \mathrm{~cm}^{2}$ [15]. A minimum surface of $0.5 \mathrm{~cm}^{2}$ was held for a score of one in this study. Choosing this cut-off value was a group decision based on interpretation of reference images in the MRI atlas. For scoring effusion in ankles the cut-off values used in knees were reduced by $50 \%$ compared to the knee according text and interpretation of reference images in the MRI atlas [15]. The applied surfaces of joint effusion with corresponding scores are shown in Table 1.

Table 1. Applied surfaces with corresponding scores of joint effusion in knees and ankles

\begin{tabular}{llll}
\hline & Knees & Ankles & \\
\hline Effusion & Surface $\left(\mathrm{cm}^{2}\right)$ & Surface $\left(\mathrm{cm}^{2}\right)$ & Score \\
Small & $0.50-1.00$ & $0.25-0.50$ & 1 \\
Moderate & $1.00-2.00$ & $0.50-1.00$ & 2 \\
Large & $>2.00$ & $>1.00$ & 3 \\
\hline
\end{tabular}

\section{Analysis}

Results of age, time of questionnaire before MRI, and joint effusion were compared between patients and controls using the Mann-Whitney U-test. The chi-square test was used to compare the frequencies of impact sports and to compare scores of joint effusion. Significant differences were stated as a $P$-value $<0.05$. A multivariate regression with gamma distribution was performed to the assess associations of joint effusion with impact sports, age, patient/control status, and MRI score. Multivariate regression analyses were assessed separately for knees and ankles.

\section{Results}

In total 104 joint (52 knees, 52 ankles) of 26 haemophilic patients and 120 joints ( 60 knees, 60 ankles) of 30 healthy young men (controls) were reassessed. One ankle of an haemophilic patient was excluded for evaluation because of previous arthrodesis. Medians of age were 21 years for haemophilic patients and 24 years for controls, which was not significantly different $(P=0.06)$. A questionnaire about sport activities had been administered in $62 \%$ of haemophilic patients in the context of other studies with a median of 2.2 years 
(range 0-3.8) before the MRI examination. All controls had a questionnaire about their sport activities on the same day as MRI examination. Median frequencies of impact sports were comparable for both groups; 1 per week for haemophilic patients and 2 per week for controls $(\mathrm{P}=0.09)$ (Table 2).

Table 2. Baseline characteristics of patients and healthy controls

\begin{tabular}{llll}
\hline & $\begin{array}{l}\text { Haemophilic patients } \\
\mathrm{n}=26 \text { patients, } \\
104 \text { joints }\end{array}$ & $\begin{array}{l}\text { Healthy controls } \\
\mathrm{n}=30 \text { controls, } \\
120 \text { joints }\end{array}$ & $\begin{array}{l}P \text {-value } \\
\end{array}$ \\
\hline Age (years) & $21[17-24]$ & $24[23-25]$ & 0.06 \\
Moderate / severe haemophilia & $10(38 \%) / 16(62 \%)$ & - & \\
On demand / prophylactic treatment & $10(38 \%) / 16(62 \%)$ & - & \\
Questionnaire about sport activities & $16(62 \%)$ & $30(100 \%)$ & $<0.01$ \\
Time before MRI (years) & $2.2[1.2-3.7]$ & $0.0[0.0-0.0]$ & 0.09 \\
Impact sports (times a week in May) & $1[0-3]$ & $2[1-3]$ & \\
\hline
\end{tabular}

Values are median [inter quartile ranges (IQR)] or $\mathrm{n}(\%)$.

Knees of controls showed significant more joint effusion compared to haemophilic patients $(P<0.01)$. The cohort of haemophilic patients had a median surface of knee effusion of $0.31 \mathrm{~cm}^{2}$ (range 0-1.08) compared to $0.68 \mathrm{~cm}^{2}$ (range 0-2.81) in the control group (Table 3). Subsequently, knees of controls scored significantly higher and had more often a positive score $(\geq 1)$ compared to knees of haemophilic patients (67\% vs. $23 \%, P>0.01)$. Surfaces and scores of effusion in ankles were comparable between haemophilic patients and controls.

For the knee a significant positive association was found between the surface of effusion and frequency of impact sports. Healthy controls showed more joint effusion than haemophilic patients. Age and MRI score showed no association with the amount of knee effusion. Very little effusion was observed in the ankle joints and no significant associations between joint effusion and age, frequency of impact sports, MRI score, or patient/control status could be observed. 
Table 3. Surfaces and scores of joint effusion on sagittal MRI in knees and ankles of healthy controls and haemophilic patients, stratified for disease severity

\begin{tabular}{lccccc}
\hline & \multicolumn{3}{c}{ Haemophilic patients } & \multirow{2}{*}{ Healthy controls } & $P$-value* \\
\cline { 2 - 4 } & Moderate & Severe & Total & & \\
\hline Knees & 20 & 32 & 52 & 60 & \\
Effusion-surface $\left(\mathrm{cm}^{2}\right)$ & $0.3[0.1-0.5]$ & $0.3[0.2-0.5]$ & $0.3[0.2-0.5]$ & $0.7[0.4-1.1]$ & $<0.01$ \\
Effusion-score & $0[0-0]$ & $0[0-0]$ & $0[0-0]$ & $1[0-2]$ & $<0.01$ \\
Score 0 & $15(75 \%)$ & $25(78 \%)$ & $40(77 \%)$ & $20(33 \%)$ & \\
Score 1 & $5(25 \%)$ & $5(16 \%)$ & $10(19 \%)$ & $22(37 \%)$ & \\
Score 2 & $0(0 \%)$ & $2(6 \%)$ & $2(4 \%)$ & $12(20 \%)$ & \\
Score 3 & $0(0 \%)$ & $0(0 \%)$ & $0(0 \%)$ & $6(10 \%)$ & \\
Ankles & 19 & 32 & 51 & 60 & 0.92 \\
Effusion - surface $\left(\mathrm{cm}^{2}\right)$ & $0.0[0.0-0.1]$ & $0[0.0-0.2]$ & $0.0[0.0-0.1]$ & $0.0[0.0-0.1]$ & \\
Effusion - score & $0[0-0]$ & $0[0-1]$ & $0[0-0]$ & $0[0-0]$ & \\
Score 0 & $16(84 \%)$ & $24(75 \%)$ & $40(78 \%)$ & $50(83 \%)$ & \\
Score 1 & $2(11 \%)$ & $4(13 \%)$ & $6(12 \%)$ & $6(10 \%)$ & \\
Score 2 & $1(5 \%)$ & $4(13 \%)$ & $5(10 \%)$ & $2(3 \%)$ & \\
Score 3 & $0(0 \%)$ & $0(0 \%)$ & $0(0 \%)$ & $2(3 \%)$ & \\
\hline
\end{tabular}

One ankle of a patient with moderate haemophilia was excluded because of previous arthrodesis. Values are median [inter quartile ranges (IQR)] or $\mathrm{n}(\%)$. Knees of controls had significantly more often a positive score $(\geq 1)$ compared to knees of haemophilic patients $(67 \%$ vs. $23 \%, P<0.01)$. ${ }^{*} P$-value for differences between healthy controls and the total of haemophilic patients.

\section{Discussion}

The present study is the first to determine the specificity of joint effusion in haemophilic patients. Although the cohort size of haemophilic patients and healthy controls was rather small, significantly more joint effusion was observed in knees of healthy controls compared to knees in haemophilic patients. As a result, positive scores for knee effusion were more common in controls compared to haemophilic patients. Effusion and scores were comparable for ankles for both groups.

The current study used the most recent IPSG MRI scale for haemophilic arthropathy which is easier in use with similar reliability compared to the previous MRI scores $[8,9,11]$. Both patients and controls were imaged with the same three Tesla MRI scanner and the same imaging sequences. However, in both groups the differences in the imaging protocol between knees and ankles were present since sagittal PDW SPAIR images for ankles were not available. Effusion in the ankle was measured on sagittal T1-weighted images which were linked with coronal PDW SPAIR images. 
Since the MRI atlas only provides clear cut-off values for scoring moderate and large effusion in knees and ankles the lower cut-off values were determined based on interpretation of reference images in the MRI atlas [15]. These cut-off values were a group decision based on interpretation of reference images. The reason to determine a minimal amount of effusion was to prevent positive scores $(\geq 1)$ in all joints with an area of fluid smaller than shown in the MRI atlas [15]. This cut-off value for a minimal amount of effusion can be discussed. However, choosing somewhat different cutoff values will not alter the statistical differences for knee effusion between patients and controls. Lowering the cut-off value from 0.50 to $0.35 \mathrm{~cm} 2$ would have resulted in $11(21 \%)$ more positive scores in patients as well as 11 $(15 \%)$ more scores positive scores in controls. Increase the cut-off from 0.50 to $0.65 \mathrm{~cm}^{2}$ would have decreased the number of positive joint scores by 6 $(12 \%)$ in patients and $8(13 \%)$ in controls. Scores for knee effusion remained significantly higher $(P<0.01)$ for controls independent from the chosen cutoff values of $0.35,0.50$ or $0.65 \mathrm{~cm}^{2}$.

Frequencies of impact sports in both cohorts were similar in this study. Therefore, bias by impact sports in the results of effusion between the two cohorts is unlikely. Correlation between impact sports and surfaces of joint effusion on sagittal MRI was analysed to evaluate whether impact sports influenced the amount of joint effusion. The observed correlation between the frequency of impact sports and surfaces of knee effusion might implicate a physiological cause of joint effusion. Though, the time of questionnaire before MRI examination was rather long and frequencies could have altered over time in haemophilic patients. Questionnaires concerning type and frequency of sports were not available for all haemophilic patients. These patients without an administered questionnaire could differ regarding sport activities.

The presence of joint effusion in healthy subjects has been reported in other studies too. Joint effusion was common in an MRI study in 134 patients to determine abnormalities in symptomatic and contralateral knees after knee trauma [16]. Effusion was scored by consensus between two radiologists, but stated criteria to define those categories of joint effusion were not reported. In symptomatic knees $50 \%$ showed small effusion compared to $47 \%$ in contralateral knees. Multivariable analysis shows that only moderate and large effusion were related to recent trauma and osteoarthritis [16], suggesting that small effusion is physiological rather than pathological. Controls with a joint 
injury within 8 weeks before the examination were excluded in our study. A study by Kolman et al. correlated joint effusion and pathological findings in 115 knees evaluated by MRI [17]. Joint effusion was determined by measuring the anterior-posterior distance of the suprapatellar pouch in the midline of the knee and in the widest point of the suprapatellar pouch in the lateral aspect of the knee. They concluded that an anterior-posterior distance of 10 $\mathrm{mm}$ or less effusion in the lateral suprapatellar pouch is physiologic based on a negative predictive value of $86 \%$, a sensitivity of $93 \%$ and a specificity of $66 \%$ for other pathological findings, e.g. moderate and severe osteoarthritis [17].

Literature about joint effusion due to sports participation supports the hypothesis that effusion might be a physiological and protective response. Ten asymptomatic joggers with averages of 25-30 km per week had an MRI of one knee before 30 minutes continuous jogging and an MRI within 10 minutes afterwards [18]. Small knee effusion according to consensus by four radiologist was seen in $40 \%$ before jogging and the amount of effusion increased after jogging. Influences of marathon running on knee effusion was studied in 22 non-professional runners [19]. Joint effusion was described as a diameter more than $1 \mathrm{~cm}$ in retropatellar bursa on sagittal dual Turbo SE MRI. In 59\% of runners effusion was present before marathon running and increased in $31 \%$ of them after running a marathon. Similar findings of the effects of marathon running are described by Lohman et al. [20]. MRI's of ankles within three hours after running a marathon showed slightly increased amounts of joint fluid in 34\% of ankles. An increased amount of joint fluid was observed in 18\% of ankles in the control group who ran 15-30 km per week on average.

MRI is the most sensitive imaging modality for assessment of joints in haemophilic patients with early stage arthropathy. A standardized MRI scale makes evaluation of prophylactic treatment strategies in haemophilia feasible. The clinical meaning of joint effusion is unclear. This study as well as previous literature suggest that joint effusion is not directly related to haemophilic arthropathy. Therefore it is impossible to make management decisions concerning treatment and sports participation based on a single observation of joint effusion. Di Minno et al. considered joint effusion as a reversible finding in their study to evaluate joints with MRI and ultrasound (US) [21]. In his recent publication on a joint US score, Martinoli et al. stated that joint effusion was not included in the score as it did not represent chronic 
arthropathy and is likely to cause fluctuations in the score since it is a transient finding [22]. Eventually Martinoli did not include effusion in the score, but included a formal documentation of effusion in the scoring system. Including joint effusion in MRI scales for haemophilic arthropathy may reduce the discriminative power of the scale, especially in the comparisons of outcome between different groups of patients. Therefore the best option for MRI may be to not include effusion in the scoring system for the evaluation of haemophilic arthropathy. Omission in the scoring system does not preclude recording of large effusions for clinical follow-up in individual cases.

\section{Conclusion}

This study shows that joint effusion is observed more frequently in knees of healthy controls than in haemophilic knees. In ankles, joint effusion was comparable between patients and controls. This suggests that joint effusion in knees and ankles is not haemophilia specific. Inclusion of joint effusion in the MRI scale could be reconsidered since it is expected to reduce the specificity of this scale for evaluating haemophilic arthropathy. 


\section{References}

1 Srivastava A, Brewer AK, Mauser-Bunschoten EP, et al. Guidelines for the management of hemophilia. Haemophilia 2013; 19: e1-47.

2 Arnold WD, Hilgartner MW. Hemophilic arthropathy. Current concepts of pathogenesis and management. J Bone Jt Surg Am 1977; 59: 287-305.

3 Aronstam A, Rainsford SG, Painter MJ. Patterns of bleeding in adolescents with severe haemophilia A. Br Med J 1979; 1: 469-70.

4 Pettersson H, Ahlberg A, Nilsson IM. A radiologic classification of hemophilic arthropathy. Clin Orthop Relat Res 1980; 149: 153-9.

5 Nuss R, Kilcoyne RF, Geraghty S, et al. MRI findings in haemophilic joints treated with radiosynoviorthesis with development of an MRI scale of joint damage. Haemophilia 2000; 6: 162-9.

6 Funk MB, Schmidt H, Becker S, et al. Modified magnetic resonance imaging score compared with orthopaedic and radiological scores for the evaluation of haemophilic arthropathy. Haemophilia 2002; 8: 98-103.

7 Lundin B, Pettersson H, Ljung R. A new magnetic resonance imaging scoring method for assessment of haemophilic arthropathy. Haemophilia 2004; 10: 383-9.

8 Lundin B, Babyn P, Doria a S, et al. Compatible scales for progressive and additive MRI assessments of haemophilic arthropathy. Haemophilia 2005; 11: 109-15.

9 Lundin B, Manco-Johnson ML, Ignas DM, et al. An MRI scale for assessment of haemophilic arthropathy from the International Prophylaxis Study Group. Haemophilia 2012; 18: 962-70.

10 Doria A, Lundin B, Kilcoyne R, et al. Reliability of progressive and additive MRI scoring systems for evaluation of haemophilic arthropathy in children: expert MRI Working Group of the International Prophylaxis Study Group. Haemophilia 2005; 11: 245-53.

11 Doria AS, Babyn PS, Lundin B, et al. Reliability and construct validity of the compatible MRI scoring system for evaluation of haemophilic knees and ankles of haemophilic children. Expert MRI working group of the international prophylaxis study group. Haemophilia 2006; 12: 503-13.

12 Kilcoyne RF, Nuss R. Radiological assessment of haemophilic arthropathy with emphasis on MRI findings. Haemophilia 2003; 9 suppl 1: 57-64.

13 Foppen W, Sluiter D, Witkamp TD, Mali WPTM, Fischer K. Haemophilic magnetic resonance imaging score in healthy controls playing sports. Haemophilia 2013; 19: 939-43.

14 Den Uijl IEM, De Schepper A, Camerlinck M, Grobbee DE, Fischer K. Magnetic resonance imaging in teenagers and young adults with limited haemophilic arthropathy: baseline results from a prospective study. Haemophilia 2011; 17: 926-30.

15 Nuss R, Kilcoyne R. The MRI Atlas of Hemophilic Arthropathy. NewYork: Professional Publishing Group, Ltd; 2002.

16 Boks SS, Vroegindeweij D, Koes BW. Magnetic Resonance Imaging Abnormalities in Symptomatic and Contralateral Knees Prevalence and Associations With Traumatic History in General Practice. Am J Sport Med 2006; 34: 1984-91.

17 Kolman BH, Daffner RH, Sciulli RL, Soehnlen MW. Correlation of joint fluid and internal derangement on knee MRI. Skeletal Radiol 2004; 33: 91-5.

18 Kursunoglu-brahm S. Jogging Causes Acute Changes in the Knee Joint: An MRI Study in Normal Volunteers. Am J Roentgenol 1990; 154: 1233-5. 
Is joint effusion on MRI specific for haemophilia?

19 Schueller-Weidekamm C, Schueller G, Uffmann M, Bader TR. Does marathon running cause acute lesions of the knee? Evaluation with magnetic resonance imaging. Eur Radiol 2006; 16: 2179-85.

20 Lohman M, Kivisaari a, Vehmas T, et al. MRI abnormalities of foot and ankle in asymptomatic, physically active individuals. Skeletal Radiol 2001; 30: 61-6.

21 Di Minno MND, Iervolino S, Soscia E, et al. Magnetic resonance imaging and ultrasound evaluation of "healthy" joints in young subjects with severe haemophilia A. Haemophilia 2013 1-7.

22 Martinoli C, Della Casa Alberighi O, di Minno G, et al. Development and definition of a simplified scanning procedure and scoring method for Haemophilia Early Arthropathy Detection with Ultrasound (HEAD-US). Thromb Haemost 2013; 109: 1-10. 


\section{CHAPTER 5}

\section{MRI changes predict joint bleeding and progression of arthropathy in haemophilia patients}




\section{Abstract}

\section{Introduction}

There is an increasing interest to use magnetic resonance imaging (MRI) to assess early joint changes in haemophilia patients. However, the clinical relevance of early MRI findings is still unknown. The aim of this prospective cohort study was to assess the predictive value of MRI for joint bleeding and progression of arthropathy on X-rays five years later.

\section{Methods}

Both knees and ankles of haemophilia patients with no or limited arthropathy on X-rays were assessed by 3Tesla MRI and X-rays. MRI-scans were scored according to the International Prophylaxis Study Group (IPSG) MRI score for haemophilic arthropathy. Patients were followed for 5 years, including assessment of joint bleeding and repeated X-ray assessment. The association between MRI findings with bleeding and progression of arthropathy were expressed as odds ratios (OR). Analyses were adjusted for type of treatment and joint (knee/ankle).

\section{Results}

Baseline assessment included 104 joints of 26 haemophilia patients with a median age of 21 years. Four ankles with severe joint changes were excluded. Follow-up was available in $92 \%$ of joints. Any joint bleeding was reported for $36 \%$ of joints. Joints with synovial hypertrophy had $80 \%$ chance of 5 -year joint bleeding, compared to $27 \%$ in joints without synovial hypertrophy (adjusted OR: 9.1, P<0.05). Any MRI finding, except joint effusion, was predictive for progression of arthropathy (positive predictive value: $75 \%$, negative predictive value $98 \%$ ).

\section{Conclusion}

Joints with synovial hypertrophy on MRI had a significantly higher change of bleeding compared to joints without synovial hypertrophy. Along with synovial hypertrophy, any MRI finding except joint effusion, was predictive for progression of arthropathy. 


\section{Introduction}

Patients with haemophilia have a high tendency to bleed due to a deficiency in coagulation factor VIII or IX (FVIII/IX). Clotting factor replacement therapy has been available since the 1960s and is nowadays the preferred treatment of patient with severe haemophilia [1,2]. Unfortunately, prophylactic replacement therapy (prophylaxis) is costly and minimum trough levels of FVIII/IX of $1-2 \%$ do not guarantee complete prevention of bleeding $[3,4]$, especially in the large joints. Prolonged or repeated joint bleeding results in synovial hypertrophy and progressive osteochondral changes $[5,6]$. As a result, arthropathy is still a common comorbidity in haemophilia patients. More intensive prophylaxis is associated with lower bleeding rates but a significant increase in treatment costs [7]. Therefore, tailoring treatment according to bleeding risk may help improve outcome as well as the cost-effectiveness of prophylaxis.

Traditionally, the six main joints (bilateral elbows, knees, and ankles) are examined with standard X-rays to monitor joint status. However, standard $\mathrm{X}$-rays are able to assess osteochondral changes only which occur late and are mostly irreversible. As treatment of haemophilia improved over the past decades, there is an increasing interest to assess early joint changes. Magnetic Resonance Imaging (MRI) can show early joint changes which are not detected by clinical examination or X-rays [8]. The clinical relevance of these early MRI changes is still not fully understood, but synovial hypertrophy is characterised by neovascularisation which is expected to increase the risk of bleeding [5,9], as well as metabolic osteochondral changes [10,11]. The aim of this prospective cohort study was to assess the predictive value of MRI changes for 5-year joint bleeding and 5-year progression of arthropathy on X-rays.

\section{Methods}

The design of this single centre prospective cohort study has been described previously [8]. In short, baseline evaluation using MRI and X-rays within 2 years of MRI was performed in 2009-2010. Follow-up with bleeding data and X-rays was performed in 2014-2015. Inclusion criteria were moderate (1-5\% FVIII/IX activity) or severe ( $<1 \%$ FVIII/IX activity) haemophilia, aged 12-30 years, with no or minimal arthropathy on X-rays. Minimal arthropathy in patients was defined as at most one knee or ankle with a 
radiological Pettersson score $\geq 4$ [12]. In general, severe haemophilia patients in our centre (VCK) receive prophylaxis tailored according to bleeding rates. Patients with moderate haemophilia are mostly treated on-demand. Based on treatment, bleeding risk was therefore assumed to be similar among patients. Exclusion criteria were inhibitor development for clotting factor concentrates or a contra-indication for MRI. The research protocol was approved by the institutional ethical review board and written informed consent was obtained from all participants. Results were reported according to the 'Strengthening the Reporting of Observational Studies in Epidemiology' (STROBE) statement $[13,14]$.

\section{Data collection}

X-rays of knees and ankles were scored by one observer, blinded for MRI findings and number of registered joint bleeds, according to the Pettersson score with use of a consensus atlas [12,15]. Baseline and follow-up X-rays were compared to assess whether progression had occurred. Progression of arthropathy on X-rays was defined as any increase in the Pettersson score.

Three Tesla MR equipment (Philips, type Achieva 3T TX; Koninklijke Philips Electronics NV, the Netherlands) was used in all patients. The protocol for the knee consisted of $2 \mathrm{~mm}$ sagittal 3D Water only Selection (WATS), a $3.5 \mathrm{~mm}$ sagittal and transversal Proton Density Weighted (PDW) Spectral Adiabatic Inversion Recovery (SPAIR) and a $3.5 \mathrm{~mm}$ coronal T1-weighted Spin echo (SE). For the ankle the imaging protocol consisted of $2 \mathrm{~mm}$ coronal 3D WATS, a $2.5 \mathrm{~mm}$ coronal PDW SPAIR and a $2.5 \mathrm{~mm}$ sagittal T1-weighted SE. MR images were scored by two radiologists, blinded for each other's scores, according to the International Prophylaxis Study Group (IPSG) MRI score and a MRI atlas for haemophilic arthropathy [16,17]. The IPGSG MRI scale for haemophilic arthropathy evaluates the presence of soft tissue changes (effusion/haemarthrosis, synovial hypertrophy, haemosiderin) and osteochondral changes (surface erosions, cysts, and cartilage degradation). Effusion and synovial hypertrophy are scored using surface measurements on a single MRI slice. However, neither the IPSG MRI score, nor the MRI atlas for haemophilic arthropathy state a minimal threshold for scoring effusion or synovial hypertrophy [16,17]. Therefore, lower cut-off values for the presence/absence of effusion and synovial hypertrophy were determined as a group decision $\left(0.50 \mathrm{~cm}^{2}\right.$ for knees, $0.25 \mathrm{~cm}^{2}$ for ankles $)$ in order to use 
objective criteria and prevent attribution of positive scores for effusion and synovial hypertrophy smaller than shown in the MRI atlas. Items of the IPSG MRI score were dichotomized as present (scores $\geq 1$ ) or absent (score $=0$ ). In case of disagreement in MRI scores between observers, consensus scores were used.

\section{Statistical methods}

Sample size was calculated for baseline comparison of X-rays and MRI though not for assessment of the predictive value of MRI findings [8]. Medians with interquartile ranges (IQR) or percentages were calculated for patient and joint characteristics. Joints with a baseline Pettersson score $\geq 4$ were excluded from the analyses. For comparison of knees and ankles at baseline, Generalized Estimating Equations (GEE) analysis for nominal data, adjusted for clustering of joints within patients, was performed.

Associations between MRI findings and any 5-year bleeding were analysed using multivariate regressing analyses (GEE) for nominal data, taking clustering of joints/patient into account. Differences in the frequency of five year bleeding between joints with and without synovial hypertrophy were compared using GEE with a negative binomial model. Analyses were adjusted for type of treatment (prophylaxis / on demand) and for type of joints (knee / ankle). Results were expressed as odds ratios (OR) with 95\% confidence intervals (CI) [18]. Joint bleed-free survival was assessed using Kaplan-Meier analyses. $P$-values $<0.05$ were considered statistically significant.

Univariate associations between MRI findings and progression of arthropathy on X-rays were stratified for knees and ankles separately. Changes on X-rays after five years were stratified by the presence of bleeding during follow-up. All analyses were performed using SPSS (IBM SPSS Statistics version 21.0, Armank, NY).

\section{Results}

Baseline results have been reported previously [8]: out of 61 eligible patients, 26 patients with haemophilia volunteered to participate. In total, 104 joints (52 knees, 52 ankles) were imaged using X-rays and 3T MRI. Four ankles with more than minimal arthropathy on X-rays at baseline (Pettersson score $\geq 4$ ) were excluded from the analyses.

Patient and joint characteristics are presented in Table 1. At baseline, patients were aged 12-29 years. Prophylaxis was given in 15/16 with severe haemophilia 
Table 1. Baseline patient and joint characteristics

\begin{tabular}{|c|c|c|c|}
\hline Patient characteristics & $(\mathrm{n}=26)$ & & \\
\hline Age in years (IQR) & $21(17-24)$ & & \\
\hline Severe haemophilia & $16(62 \%)$ & & \\
\hline Prophylactic treatment & $16(62 \%)$ & & \\
\hline Joint characteristics & Total $(\mathrm{n}=100)$ & Knees $(\mathrm{n}=52)$ & Ankles $(\mathrm{n}=48)$ \\
\hline History of bleeding & $82(82 \%)$ & $40(77 \%)$ & $42(88 \%)$ \\
\hline $\mathrm{X}$-ray abnormalities a & $7(7 \%)$ & $2(4 \%)$ & $5(10 \%)$ \\
\hline MRI abnormalities ${ }^{b}$ & $39(39 \%)$ & $15(29 \%)$ & $24(50 \%)$ \\
\hline MRI findings & Total $(\mathrm{n}=100)$ & Knees $(\mathrm{n}=52)$ & Ankles $(\mathrm{n}=48)$ \\
\hline Effusion & $23(23 \%)$ & $12(23 \%)$ & $11(23 \%)$ \\
\hline Synovial hypertrophy & $16(16 \%)^{*}$ & $2(4 \%)$ & $14(29 \%)$ \\
\hline Haemosiderin & $16(16 \%) *$ & $2(4 \%)$ & $14(29 \%)$ \\
\hline Surface erosions & $7(7 \%)$ & $2(4 \%)$ & $5(10 \%)$ \\
\hline Subchondral cysts & $5(5 \%)$ & $1(2 \%)$ & $4(8 \%)$ \\
\hline Cartilage degradation & $9(9 \%)$ & $3(6 \%)$ & $6(13 \%)$ \\
\hline
\end{tabular}

IQR, inter quartile range. ${ }^{a}$ Pettersson score $\geq 1$. ${ }^{\mathrm{b}}$ IPSG MRI score $\geq 1$. * Significant differences between knees and ankles $(P<0.01$; adjusted for clustering of joint within patients).

(weekly dose: $38 \mathrm{IU} / \mathrm{kg}$, IQR: 23-44) and in 1/8 patients with moderate haemophilia. Treatment remained unchanged during follow-up. None of the patients was treated for a clinical synovitis at baseline. The majority of joints $(82 \%)$ had a history of bleeding before MRI (median lifetime number of joint bleeds/joint: 2, IQR 1-5). Although X-ray abnormalities were observed in $7 \%$ of joints only, $38 \%$ of joints showed MRI abnormalities (Table 1). The most prevalent MRI findings were effusion (23\%), synovial hypertrophy $(16 \%)$, and haemosiderin (16\%). Synovial hypertrophy and haemosiderin were significantly more prevalent in ankles compared to knees $(P<0.01)$.

Follow-up of patients is shown in Figure 1, missing data on 5-year bleeding or follow-up X-rays was $<10 \%$. After five years (IQR: 4.5-5.4, range: 2.8 6.1), two patients ( $8 \%$ of joints) were lost to follow-up for X-rays. Two other patients ( $8 \%$ of joints) were lost to follow-up for bleeding data because of unreliable bleeding registries and/or emigration. 


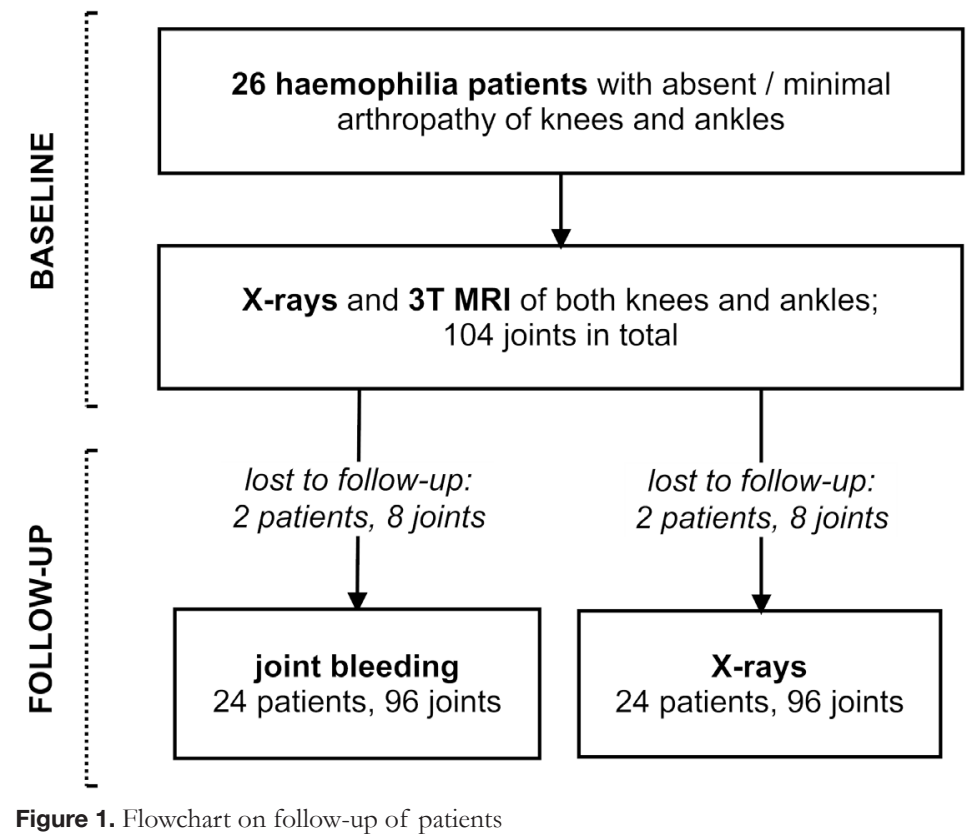

\section{Five year bleeding}

The majority of patients (88\%) experienced knee or ankle bleeds during follow-up (median number of bleeds in knees and ankles combined: 3, IQR: 1-4). A total of $36 \%$ of joints (CI: 26-47) was exposed to bleeds during follow-up: 11 knees and 22 ankles had joint bleeds within five years after MRI. Severity of haemophilia and type of treatment were not associated with five year bleeding.

Associations of MRI findings with five year bleeding are presented in Table 2. MRI findings of synovial hypertrophy and haemosiderin were the strongest and only significant predictors for five year bleeding. Analyses adjusted for type of treatment and type of joint (knee or ankle) showed an independent increased risk of five year bleeding for joints with synovial hypertrophy (adjusted OR: 9.1, CI: 2.6-31.4). Sensitivity analyses using multiple imputation yielded similar results (Table S1). As MRI findings of synovial hypertrophy and haemosiderin were collinear (phi-coefficient $0.85, P<0.01$ ), the independent associations of these MRI findings could not be assessed. 
Table 2. Associations of MRI findings with five year bleeding

\begin{tabular}{lcc}
\hline MRI findings & Univariate & Adjusted for treatment and joint \\
\hline Effusion & $1.0(0.3-2.7)$ & $1.1(0.4-3.2)$ \\
Synovial hypertrophy & $10.7(2.7-41.6)^{*}$ & $9.1(2.6-31.4)^{*}$ \\
Haemosiderin & $10.7(2.7-41.6)^{*}$ & $8.9(2.6-30.9)^{*}$ \\
Surface erosions & $5.1(0.9-27.9)$ & $4.8(0.5-42.6)$ \\
Subchondral cysts & $8.0(0.9-74.9)$ & $7.3(0.6-91.2)$ \\
Cartilage degradation & $4.2(0.9-17.9)$ & $4.1(0.9-17.7)$ \\
\hline
\end{tabular}

Values are odds ratios with $95 \%$ confidence intervals. $*$ Significant predictor for five year bleeding.

Further analyses were performed to assess the absolute risk of bleeding in joints with synovial hypertrophy. Joint bleed-free survival after MRI assessment was significantly worse for knees and ankles with MRI synovial hypertrophy compared to joint without synovial hypertrophy $(P<0.01)$, as shown in Figure 2A. The absolute risk of any 5 -year bleeding was 80\% (CI: $52-96 \%$ ) for joints with synovial hypertrophy and $27 \%$ (CI: 18-39) for joints without synovial hypertrophy. Joints with synovial hypertrophy bled earlier and more frequently: median number of 5-year bleeds was 2 (IQR: 1-3, range $0-8$ ) for joints with synovial hypertrophy versus a median of 0 (IQR: $0-1$, range: $0-4)$ for joints without synovial hypertrophy $(P<0.01)$.

A Bleed-free survival at joint level

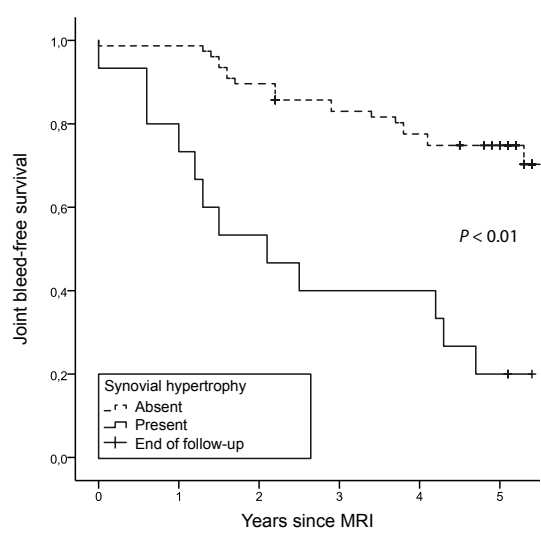

B Bleed-free survival at patient level

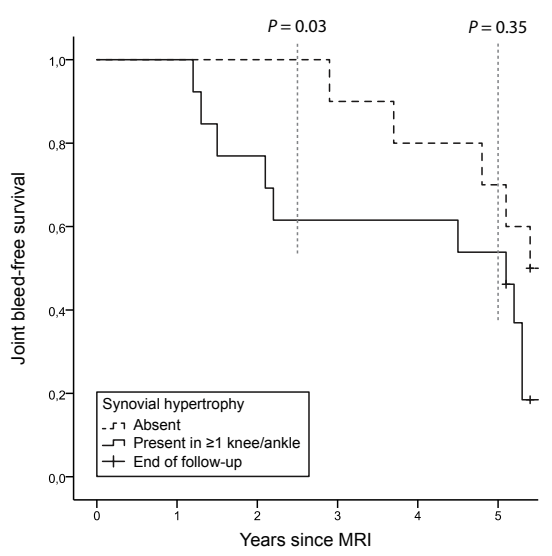

Figure 2. Joint bleed-free survival after MRI assessment for joint with- and without synovial hypertrophy A) The absolute risk of any 5 -year bleeding was $80 \%$ for joints with synovial hypertrophy and $27 \%$ for joints without synovial hypertrophy. B) Patients with synovial hypertrophy in $\geq 1$ joints had an absolute risk of 2.5-year joint bleeding of $39 \%$ while patient without any synovial hypertrophy did not experience joint bleeding in 2.5 years. The 5 -year bleeding risk was comparable in both patient groups. 
The finding of synovial hypertrophy on MRI could be used to stratify patients according to their 2.5-year bleeding risk as shown in Figure 2B. The absolute risk of 2.5-year joint bleeding was 39\% for patients with synovial hypertrophy in $\geq 1 \mathrm{knee} /$ ankle and $0 \%$ for patient without any synovial hypertrophy. However, the 5-year bleeding risk was comparable among patients with/ without any synovial hypertrophy in knees or ankles.

\section{Progression of arthropathy}

Median time between series of X-rays was 5.1 years (IQR: 4.6-5.6). Changes on $\mathrm{X}$-rays were observed in one knee and seven ankles; $8 / 92$ joints in total (9\%, CI: 4-16). Therefore only univariate analyses were performed for the association of MRI characteristics with progression of arthropathy on X-rays. Observed changes on X-rays were narrowing of joint space, irregularity of subchondral bone, and/or subchondral cyst formation. Severity of haemophilia and type of treatment were not associated with progression of arthropathy. Baseline X-ray abnormalities were predictive for progression of arthropathy five years later (OR 6.7, CI: 1.0-44.1) although MRI findings had a stronger association with progression of arthropathy (OR 13.3, CI: 1.6-113.2).

Associations of MRI findings with progression of arthropathy are presented in Table 3. Joints with effusion on MRI showed X-rays changes in $4 \%$ only (CI: 0-22). Effusion was therefore not associated with progression of arthropathy (OR 0.5, 0.1-3.9). All MRI findings of synovial hypertrophy, haemosiderin, surface erosions, cysts, and/or cartilage degradation showed a significant association with progression of arthropathy.

Table 3. Associations of MRI findings with progression of arthropathy, stratified for knees and ankles

\begin{tabular}{lccc}
\hline MRI findings & Total & Knees & Ankles \\
\hline Effusion & $0.5(0.1-3.9)$ & a & b \\
Synovial hypertrophy & $13.9(2.8-68.1)$ & a & $10.7(1.7-67.1)$ \\
Haemosiderin & $28.5(4.9-165.4)$ & $a^{a}$ & $31.0(3.1-306.2)$ \\
Surface erosions & $41.0(5.7-294.6)$ & a & $27.0(2.2-324.9)$ \\
Subchondral cysts & $13.7(1.6-114.8)$ & a & $14.4(1.1-189.3)$ \\
Cartilage degradation & $20.0(3.6-110.7)$ & a & $13.1(1.7-103.7)$ \\
\hline
\end{tabular}

Values are odds ratios with $95 \%$ confidence intervals. Odds ratios could not be calculated as cross tables included a zero: ${ }^{\text {a }}$ Progression of arthropathy was observed in one knee only; ${ }^{\mathrm{b}}$ Progression of arthropathy was not observed ankles with effusion. 
In addition to MRI findings, joint bleeding may contribute to progression of arthropathy. Changes on X-rays after five years were therefore stratified by the presence of MRI findings and bleeding during follow-up in Figure 3. MRI findings were observed in 4/70 apparently normal joints (6\%, CI: 2-14). Changes on X-ray after 5 years were observed in $3 / 4$ joints with MRI findings (positive predictive value: 75\%, CI: 19-99). Progression of arthropathy on $\mathrm{X}$-rays was rarely observed in joint without MRI abnormalities at baseline (negative predictive value: 98\%, CI: 92-100). The sensitivity analyses showed similar risk associations between MRI findings and progression of arthropathy (Table S2, Figure S1).

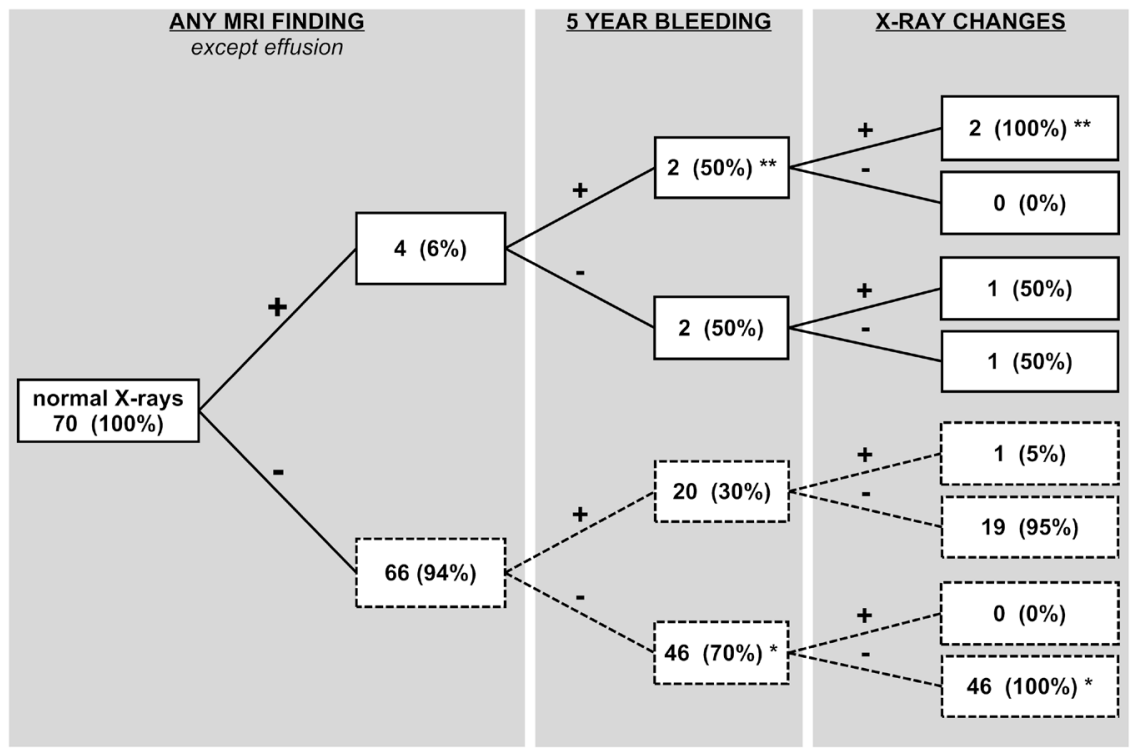

Figure 3. Observed development of X-ray changes in apparently normal joints, stratified by the presence of MRI findings and reported bleeding during five year follow-up

+ present; - absent. Complete case analyses for patients with data on five year bleeding and available follow-up X-rays. Joints with baseline MRI findings (excluding joint effusion) had the highest chance of progression of arthropathy: $3 / 4$ joints $(75 \%)$ showed X-ray changes after five years. Joints without MRI findings showed X-ray changes in 1/66 (2\%) only. Two joints with MRI findings at baseline developed a clinical synovitis (**) after bleeding and showed X-ray changes as well. One other joint developed a clinical synovitis $\left(^{*}\right)$ though was normal on baseline MRI, did not experience bleeds, and was normal on follow-up X-rays. 


\section{Discussion}

The present study showed that MRI findings are predictive for 5-year joint bleeding and progression of arthropathy in haemophilia patients with limited arthropathy. Joints with synovial hypertrophy bled sooner and more often compared to joint without synovial hypertrophy. Along with synovial hypertrophy, any IPSG MRI finding except joint effusion, appeared to be associated with progression of arthropathy.

Haemophilic arthropathy was assessed using standardized imaging scores like the Pettersson score and IPSG MRI score $[12,16]$. In this study MRI was performed without the administration of contrast. MRI without contrast is less sensitive compared to MRI with contrast for the detection of synovial hypertrophy in juvenile idiopathic arthritis (JIA) [19,20]. Therefore, the prevalence of subtle synovial hypertrophy may have been underestimated in our study. In contrast with the report on JIA, synovial hypertrophy in haemophilia was scored with less detail, using surface measurements on a single MRI slice [19,29]. Although synovial hypertrophy in haemophilia patients is better delineated on MRI with contrast, the presence/absence of synovial hypertrophy was scored similar on MRI with- and without contrast [21]. As synovial hypertrophy on MRI without contrast identified joints with a high and low bleeding risk already very accurately in the current study, the additional value of MRI with contrast is expected to be limited.

The present study had sufficient power for multivariate analyses to assess the association of MRI findings with 5-year bleeding. Progression of arthropathy was observed in eight joints only, therefore allowing univariate statistical analyses only. Missing data on 5-year bleeding or follow-up X-rays was $<10 \%$ only and additional sensitivity analyses showed that this did not affect our results. Prolonged follow-up and/or use of more sensitive imaging modalities may reveal a higher incidence of progression of arthropathy and allow the multivariate analysis of its predictors.

\section{Comparison with other studies}

Patients in the present study were exposed to a median of 3 joint bleeds (sum of knees and ankles) during 5-year follow-up. This baseline bleeding risk on prophylaxis was comparable with other clinical studies reporting a median of 1.4 joint bleeds/patient/year in adolescents and 1.1 joint bleeds/patient/ years in adults [22-24]. These findings would result in a total of 6-7 joint 
bleeds/patient in five years. The bleeding data include all joints (mainly the elbows, knees, and ankles [25]), therefore approximately 4 bleeds are expected in knees and ankles only.

Although prospective studies with baseline MRI assessment were performed previously, the association of MRI findings with bleeding or progression of arthropathy has not been reported yet [26,27]. Children with haemophilia (aged $11 \pm 4$ years, 67\% on prophylaxis) were followed for four years after MRI assessment by Pergantou et al. Progression of arthropathy on X-rays was observed in $15 \%$ of joints. In contrast, improvement of X-ray scores was observed in $40 \%$ of joints (osteoporosis, enlargement of epiphysis) and may be explained by the MRI guided treatment changes in 18/40 patients [26]. Improvement of X-ray scores was not observed in our study, possibly because we observed baseline X-ray abnormalities in 7\% of joints only. Olivieri et al. studied the natural course of arthropathy in children and adolescents with haemophilia, including $88 \%$ on prophylaxis. Asymptomatic ankles (according to the World Federation of Haemophilia Orthopaedic Joint Score [28]) of 26 patients were examined using MRI and were followed for 2-8 years. Development of severe cartilage and bone defects was observed in 2/26 ankles (8\%, CI: 1-25). Synovial hypertrophy and/or mild (sub)chondral changes were observed in two other ankles. As synovial hypertrophy and mild (sub)chondral changes were not reported separately, the exact percentage of joints with osteochondral changes could not be calculated. However, the percentage of joints with progression of bony changes was 8-15\% [27]. These findings are in line with the present study.

\section{Clinical implications}

This study did not assess the aetiology of bleeding and arthropathy in haemophilia patients but aimed to evaluate the relevance of early MRI findings. Effusion on MRI was not associated with bleeding or joint changes. However, any other findings according to haemophilia MRI scores are associated with progression of arthropathy. Normal joints on MRI had a minimal risk of developing 5-year X-ray changes (negative predictive value 98\%). Synovial hypertrophy showed the strongest association with 5-year bleeding. Joints with a high risk of bleeding could be identified by the presence/absence of synovial hypertrophy on MRI: the 5-year bleeding risk was $80 \%$ for joints with synovial hypertrophy versus $27 \%$ for joints without 
synovial hypertrophy. Detection of synovial hypertrophy was associated with an increased bleeding risk at patient level too. Patients with at least one knee/ankle with synovial hypertrophy bled earlier than patients without synovial hypertrophy. This knowledge may be used to emphasize adherence to prophylaxis, or to guide treatment decisions. In patients considering to discontinue prophylaxis [29], imaging of synovial hypertrophy may be used to assess subsequent bleeding risk. As MRI is not suitable for routine joint assessment due to limited availability and costs, ultrasound may be used as an accurate alternative [30,31]. Further studies are required to confirm the associations of MRI findings with bleeding and progression of arthropathy. It remains to be established whether MRI findings are responsive to treatment changes and if prophylaxis may be tailored using imaging.

\section{Conclusion}

MRI evaluation provides relevant information in haemophilia patients with absent or limited arthropathy. Synovial hypertrophy on MRI predicts an increased 5-year bleeding risk and progression of arthropathy. Ostoeochondral changes on MRI showed an even stronger association with 5-year progression of arthropathy. Effusion on MRI was not associated with bleeding or joint disease. Although the results of this single centre study are promising, further research is required to confirm our finding and the effect of MRI-guided treatment changes. 


\section{References}

1 Pool JG, Shannon AE. Production of high-potency concentrates of antihemophilic globulin in a closed-bag system. N Engl J Med 1965; 273: 1443-7.

2 Srivastava A, Brewer AK, Mauser-Bunschoten EP, et al. Guidelines for the management of hemophilia. Haemophilia 2013; 19: e1-47.

3 den Uijl I, Biesma D, Grobbee D, Fischer K. Turning severe into moderate haemophilia by prophylaxis: are we reaching our goal? Blood Transfus 2013; 11: 364-9.

4 Ahnström J, Berntorp E, Lindvall K, Björkman S. A 6-year follow-up of dosing, coagulation factor levels and bleedings in relation to joint status in the prophylactic treatment of haemophilia. Haemophilia 2004; 10: 689-97.

5 Jansen NW, Roosendaal G, Lafeber FP. Understanding haemophilic arthropathy: an exploration of current open issues. Br J Haematol 2008; 143: 632-40.

6 Lafeber FPJG, Miossec P, Valentino L a. Physiopathology of haemophilic arthropathy. Haemophilia 2008; 14: 3-9.

7 Fischer K, Steen Carlsson K, Petrini P, et al. Intermediate-dose versus high-dose prophylaxis for severe hemophilia: comparing outcome and costs since the 1970s. Blood 2013; 122: 1129-36.

8 Den Uijl IEM, De Schepper A, Camerlinck M, Grobbee DE, Fischer K. Magnetic resonance imaging in teenagers and young adults with limited haemophilic arthropathy: baseline results from a prospective study. Haemophilia 2011; 17: 926-30.

9 Bhat V, Olmer M, Joshi S, et al. Vascular remodeling underlies rebleeding in hemophilic arthropathy. Am J Hematol 2015; 00: (in press).

10 Roosendaal G, Vianen ME, Wenting MJ, et al. Iron deposits and catabolic properties of synovial tissue from patients with haemophilia. J Bone Joint Surg Br 1998; 80: 540-5.

11 Roosendaal G, TeKoppele JM, Vianen ME, et al. Blood-induced joint damage: A canine in vivo study. Arthritis Rheum 1999; 42: 1033-9.

12 Pettersson H, Ahlberg A, Nilsson IM. A radiologic classification of hemophilic arthropathy. Clin Orthop Relat Res 1980; 149: 153-9.

13 von Elm E, Altman DG, Egger M, et al. The Strengthening the Reporting of Observational Studies in Epidemiology (STROBE) Statement: Guidelines for Reporting Observational Studies. Epidemiology 2007; 18: 800-4.

14 Vandenbroucke JP, von Elm E, Altman DG, et al. Strengthening the Reporting of Observational Studies in Epidemiology (STROBE): Explanation and Elaboration. Epidemiology 2007; 18: 805-35.

15 Foppen W, van der Schaaf IC, Beek FJ a., Verkooijen HM, Fischer K. Scoring haemophilic arthropathy on X-rays: improving inter- and intra-observer reliability and agreement using a consensus atlas. Eur Radiol 2015 (in press).

16 Lundin B, Manco-Johnson ML, Ignas DM, et al. An MRI scale for assessment of haemophilic arthropathy from the International Prophylaxis Study Group. Haemophilia 2012; 18: 962-70.

17 Nuss R, Kilcoyne R. The MRI Atlas of Hemophilic Arthropathy. NewYork: Professional Publishing Group, Ltd; 2002.

18 Steyerberg EW, Pencina MJ, Lingsma HF, et al. Assessing the incremental value of diagnostic and prognostic markers: A review and illustration. Eur J Clin Invest 2012; 42: 216-28. 
19 Hemke R, Van Rossum M a J, Van Veenendaal M, et al. Reliability and responsiveness of the Juvenile Arthritis MRI Scoring (JAMRIS) system for the knee. Eur Radiol 2013; 23: 1075-83.

20 Hemke R, Kuijpers TW, Van Den Berg JM, et al. The diagnostic accuracy of unenhanced MRI in the assessment of joint abnormalities in juvenile idiopathic arthritis. Eur Radiol 2013; 23: 1998-2004.

21 Lundin B, Berntorp E, Pettersson H, et al. Gadolinium Contrast Agent is of Limited Value for Magnetic Resonance Imaging Assessment of Synovial Hypertrophy in Hemophiliacs. Acta Radiol 2007; 48: 520-30.

22 Tarantino MD, Collins PW, Hay CRM, et al. Clinical evaluation of an advanced category antihaemophilic factor prepared using a plasma/albumin-free method: pharmacokinetics, efficacy, and safety in previously treated patients with haemophilia A. Haemophilia 2004; 10: 428-37.

23 Blanchette V, Shapiro A, LiesnerHernández R, et al. Plasma and albumin-free recombinant factorVIII: pharmacokinetics, efficacy and safety in previously treated pediatric patients. J Thromb Haemost 2008; 6: 1319-26.

24 Fischer K, Collins P, Björkman S, et al. Trends in bleeding patterns during prophylaxis for severe haemophilia: observations from a series of prospective clinical trials. Haemophilia 2011; 17: 433-8.

25 Aronstam A, Rainsford SG, Painter MJ. Patterns of bleeding in adolescents with severe haemophilia A. Br Med J 1979; 1: 469-70.

26 Pergantou H, Platokouki H, Matsinos G, et al. Assessment of the progression of haemophilic arthropathy in children. Haemophilia 2010; 16: 124-9.

27 Olivieri M, Kurnik K, Pfluger T, Bidlingmaier C. Identification and long-term observation of early joint damage by magnetic resonance imaging in clinically asymptomatic joints in patients with haemophilia A or B despite prophylaxis. Haemophilia 2012; 18: 369-74.

28 Gilbert MS. Prophylaxis: musculoskeletal evaluation. Semin Hematol 1993; 30: 3-6.

29 Nijdam A, Foppen W, de Kleijn P, et al. Discontinuing early prophylaxis in young adults with severe haemophilia A: deterioration of joint status after 10 years despite low bleeding rates. $\mathrm{J}$ Thromb Haemost 2015; 13 s2: 132-3.

30 Sierra Aisa C, Lucía Cuesta JF, Rubio Martínez A, et al. Comparison of ultrasound and magnetic resonance imaging for diagnosis and follow-up of joint lesions in patients with haemophilia. Haemophilia 2014; 20: e51-7.

31 Doria AS, Keshava SN, Mohanta A, et al. Diagnostic Accuracy of Ultrasound for Assessment of Hemophilic Arthropathy: MRI Correlation. Am J Roentgenol 2015; 204: W336-47. 
MRI changes predict joint bleeding and progression of arthropathy

\section{Supplementary material}

Table S1. Associations of MRI findings with five year bleeding using pooled estimates for missing data

\begin{tabular}{lcc}
\hline MRI findings & Univariate & Adjusted for treatment and joint \\
\hline Effusion & $1.1(0.5-2.9)$ & $1.3(0.4-3.7)$ \\
Synovial hypertrophy & $8.7(2.3-32.9) *$ & $7.0(2.1-23.7) *$ \\
Haemosiderin & $8.7(2.3-32.9) *$ & $6.8(2.0-23.5)^{*}$ \\
Surface erosions & $4.0(0.7-21.5)$ & $3.6(0.4-35.5)$ \\
Subchondral cysts & $6.2(0.7-58.3)$ & $5.8(0.1-75.5)$ \\
Cartilage degradation & $3.2(0.8-13.6)$ & $3.1(0.6-14.2)$ \\
\hline
\end{tabular}

Values are odds ratios with $95 \%$ confidence intervals. $*$ Significant association with five year bleeding Missing data analysis assumed data to be missing at random. Using multiple imputation, 10 copies of the data were derived. Pooled estimates were used for re-analyses of de predictive value of MRI findings for five year bleeding.

Table S2. Associations of MRI findings with progression of arthropathy using pooled estimates for missing data

\begin{tabular}{lccc}
\hline MRI findings & Total & Knees & Ankles \\
\hline Effusion & $0.5(0.1-3.9)$ & $\mathrm{a}$ & $8.8(1.5-53.7)$ \\
Synovial hypertrophy & $12.3(2.6-58.6)$ & $\mathrm{a}$ & $8.9(1.5-53.7)$ \\
Haemosiderin & $24.6(4.4-138.7)$ & $\mathrm{a}$ & $24.8(2.6-235.6)$ \\
Surface erosions & $29.7(4.9-179.7)$ & $\mathrm{a}$ & $14.6(1.9-115.2)$ \\
Subchondral cysts & $9.9(1.4-71.0)$ & $\mathrm{a}$ & $7.8(0.9-68.3)$ \\
Cartilage degradation & $17.4(3.3-90.9)$ & $\mathrm{a}$ & $9.5(1.4-63.7)$ \\
\hline
\end{tabular}

Values are odds ratios with $95 \%$ confidence intervals. Odds ratios could not be calculated as cross tables included a zero: a Progression of arthropathy was observed in one knee only

Missing data analysis assumed data to be missing at random. Using multiple imputation, 10 copies of the data were derived. Pooled estimates were used for re-analyses of de predictive value of MRI findings for five year progression of arthropathy. 


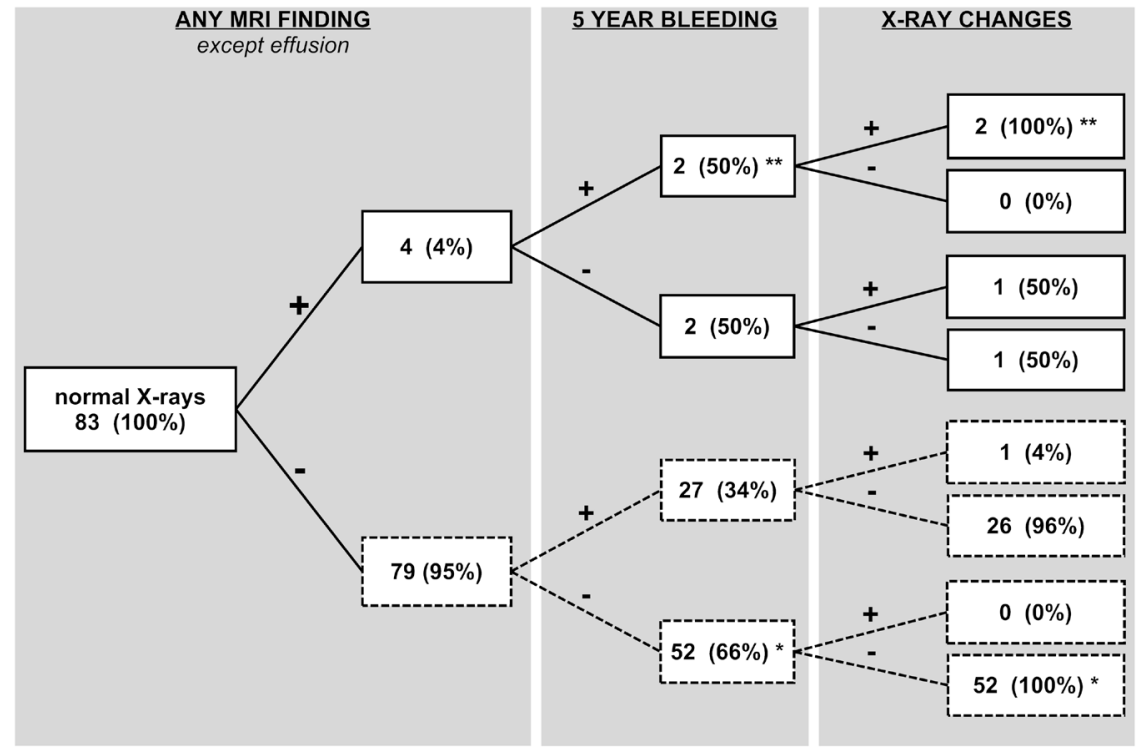

Figure S1. Observed development of X-ray changes in apparently normal joints using pooled estimates for missing data

+ present; - absent. Joints with baseline MRI findings (excluding joint effusion) had the highest chance of progression of arthropathy: $3 / 4$ joints $(75 \%)$ showed X-ray changes after five years. Joints without MRI findings showed X-ray changes in 1/66 (2\%) only. Two joints with MRI findings at baseline developed a clinical synovitis $(* *)$ after bleeding and showed X-ray changes as well. One other joint developed a clinical synovitis $\left({ }^{*}\right)$ though was normal on baseline MRI, did not experience bleeds, and was normal on follow-up X-rays. 



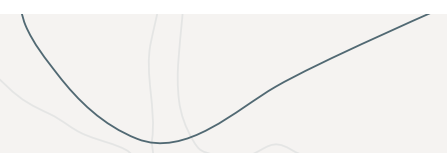

Part III

\section{Point-of-care ultrasound}

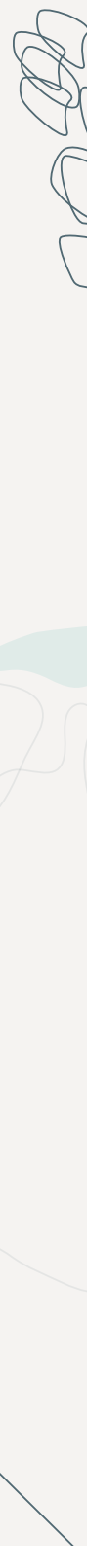


CHAPTER 6

\section{Point-of-care ultrasound in haemophilic arthropathy: diagnostic accuracy compared to MRI}




\section{Abstract}

\section{Introduction}

Recurrent joint bleeding is the hallmark of haemophilia. Synovial hypertrophy observed with Magnetic Resonance Imaging (MRI) is associated with an increased risk of future joint bleeding. The aim of this study was to investigate whether point-of-care ultrasound (POC-US) is an accurate alternative for MRI.

\section{Methods}

In a single centre study, bilateral knees and ankles of haemophilia patients with no or minimal arthropathy on X-rays were scanned using POC-US and 3 Tesla MRI. POC-US was performed by one medical doctor, blinded for MRI, according to the 'Haemophilia Early Arthropathy Detection with Ultrasound' (HEAD-US) protocol. MRIs were independently scored by two radiologists, blinded for clinical data and ultrasound results. Diagnostic accuracy parameters were calculated with $95 \%$ confidence intervals (CI).

\section{Results}

Knees and ankles of 24 haemophilia patients (96 joints), aged 18-31, were studied. Synovial hypertrophy on MRI was observed in $20 \%$ of joints. POCUS for synovial tissue was correct (accuracy) in 97\% (CI: 91-99) with a positive predictive value of 94\% (CI: 73-100) and a negative predictive value of 97\% (CI: 91-100). The overall accuracy of POC-US for cartilage defects was 91\% (CI 83-96) and for bone defects 97\% (91-99).

\section{Conclusion}

POC-US could accurately assess synovial hypertrophy, bone defects and cartilage defects in haemophilia patients with limited joint disease. POCUS may replace MRI in assessment of early joint changes and help to tailor haemophilia treatment. 


\section{Introduction}

Due to low levels of coagulation factor VIII or IX, haemophilia is characterized by repeated joint bleeding. Prophylactic clotting factor replacement therapy (prophylaxis) to reduce bleeding is the preferred treatment for patients with severe haemophilia [1,2]. Current prophylaxis regimens are insufficient to prevent all bleeding episodes. Repeated traumatic or spontaneous joint bleeds result in synovial hypertrophy and progressive osteochondral changes due to mechanical and metabolic processes [3,4]. Synovial hypertrophy on MRI is not observed in all joints with a history of bleeding [5] and may be responsive to treatment [6]. As synovial hypertrophy is associated with an increased risk of bleeding [7], early detection may be relevant in order to guide treatment changes.

Magnetic Resonance Imaging (MRI) is the most sensitive imaging modality to demonstrate early intra-articular joint changes [8]. As MRI is relatively expensive and time consuming, routine assessment of multiple joints of haemophilia patients is not feasible. As ultrasound showed to be highly accurate in assessing synovial hypertrophy, it may provide an alternative for MRI [9,10]. Various clinicians perform ultrasound examinations themselves for specific indications, known as point-of-care ultrasound (POC-US) [11]. Recently, the 'Haemophilia Early Arthropathy Detection with Ultrasound' (HEAD-US) protocol was developed to allow joint assessment in haemophilia by non-radiologists [12]. However, the diagnostic accuracy of this POC-US protocol has not been evaluated yet. The primary objective of this study was to assess the diagnostic accuracy of POC-US for synovial hypertrophy in haemophilia patients by comparison with MRI. Secondary objectives were to assess the diagnostic accuracy of POC-US for the presence or absence of cartilage and bone defects.

\section{Methods}

This diagnostic accuracy study was performed at the Van Creveldkliniek, UMCU Utrecht, the Netherlands, and reported according to the 'Standards for the Reporting of Diagnostic accuracy studies' (STARD) [13]. The research protocol was approved by the institutional ethical review board and written informed consent was obtained from all patients. 


\section{Participants}

An established imaging cohort of male adult haemophilia patients, born between 1984-1995, was invited for this cross-sectional diagnostic accuracy study [5]. Inclusion criteria were moderate or severe haemophilia ( $\leq 5 \%$ factor VIII or IX activity) and at most one joint with moderate-severe arthropathy on X-rays (Pettersson score $\geq 4$ [14]). Exclusion criteria were a history of inhibitors for clotting factor concentrates or a contraindication for MRI. Additional haemophilia patients fulfilling the same in- and exclusion criteria were invited.

\section{Imaging}

Bilateral knees and ankles of patients were examined by both POC-US and MRI. The index test was POC-US according to the Haemophilia Early Arthropathy Detection with UltraSound (HEAD-US) scanning protocol and score [12]. According to the protocol, synovial tissue was assessed in the major joint recesses. Cartilage and bone defects were assessed at one surface per joint: the femoral condyles in the knees and the talar dome in the ankles. A single ultrasound scanner (Esaote, type MyLab25 Gold, Italy) with a linear probe (adjustable central frequency at $7.5 \mathrm{MHz}, 10 \mathrm{MHz}$, and $12 \mathrm{MHz}$ ) was used. POC-US was performed by one medical doctor who received repeated training and scanned over 200 joints accordingly before the start of this study. POC-US was performed without knowledge of MRI results.

The reference test was 3 Tesla MRI (Philips, type Achieva 3T TX; Koninklijke Philips Electronics NV, the Netherlands). The MRI protocol for the knee consisted of $2 \mathrm{~mm}$ sagittal 3D Water only Selection (WATS), a $3.5 \mathrm{~mm}$ sagittal and transversal Proton Density Weighted (PDW) Spectral Adiabatic Inversion Recovery (SPAIR) and a $3.5 \mathrm{~mm}$ coronal T1-weighted Spin echo (SE) using a 16-Channel Knee Coil. For the ankle the imaging protocol consisted of 2 $\mathrm{mm}$ coronal 3D WATS, a $2.5 \mathrm{~mm}$ coronal PDW SPAIR and a $2.5 \mathrm{~mm}$ sagittal T1-weighted SE using a send-receive coil. MR images were scored by two radiologists, blinded for the ultrasound findings, according to the International Prophylaxis Study Group (IPSG) MRI scale [15]. Inconclusive results were discussed until consensus in separate meetings. Synovial hypertrophy is scored using surface measurements on a single MRI slice. However, neither the IPSG MRI score, nor the MRI atlas for haemophilic arthropathy state lower cut-off values $[15,16]$. Lower cut-off values for the presence/absence of synovial hypertrophy were determined as a group decision $\left(0.50 \mathrm{~cm}^{2}\right.$ for 
knees, $0.25 \mathrm{~cm}^{2}$ for ankles) in order to use objective criteria and prevent attribution of positive scores for synovial hypertrophy smaller than shown in the MRI atlas.

\section{Sample size}

The sample size was based on our aim to establish the diagnostic accuracy of POC-US for synovial hypertrophy. For clinical interpretation, the positive and negative predictive values are the most valuable diagnostic accuracy parameters. Based on preliminary data, the prevalence of synovial hypertrophy in the imaging cohort was estimated at $23 \%$ [5]. Based on recent data on a different ultrasound protocol [9], a sensitivity of $90 \%$ and a specificity of 95\% were expected with POC-US. The minimal acceptable positive and negative predictive values were considered to be $\geq 70 \%$. Including at least 24 patients would therefore suffice to detect a positive predictive value of $91 \%$ (95\% confidence interval (CI): 71-99) and a negative predictive value of 97\% (CI: 91-100).

\section{Analysis}

Medians with interquartile ranges (IQR) or percentages were calculated for patient and joint characteristics. POC-US and MRI scores of synovial hypertrophy, cartilage defects, and bone defects were dichotomized as absent $(0)$ or present $(\geq 1)$. Calculated diagnostic accuracy parameters with 95\% CI were the sensitivity, specificity, positive predictive value, negative predictive value, and percentage correctly identified (accuracy). Analyses were performed using SPSS (IBM SPSS Statistics version 21.0, Armank, NY).

\section{Results}

In total, 96 joints of 24 patients were included between November 2014 and August 2015. Seven of the 26 patients of the original imaging cohort were unwilling to participate in the current study. Five additional patients fulfilling the same inclusion criteria were included to reach the inclusion target of 24 patients. All patients underwent POC-US (index test) and MRI (reference test) as shown in Figure 1. One ankle with ankylosis was excluded in the analyses. 


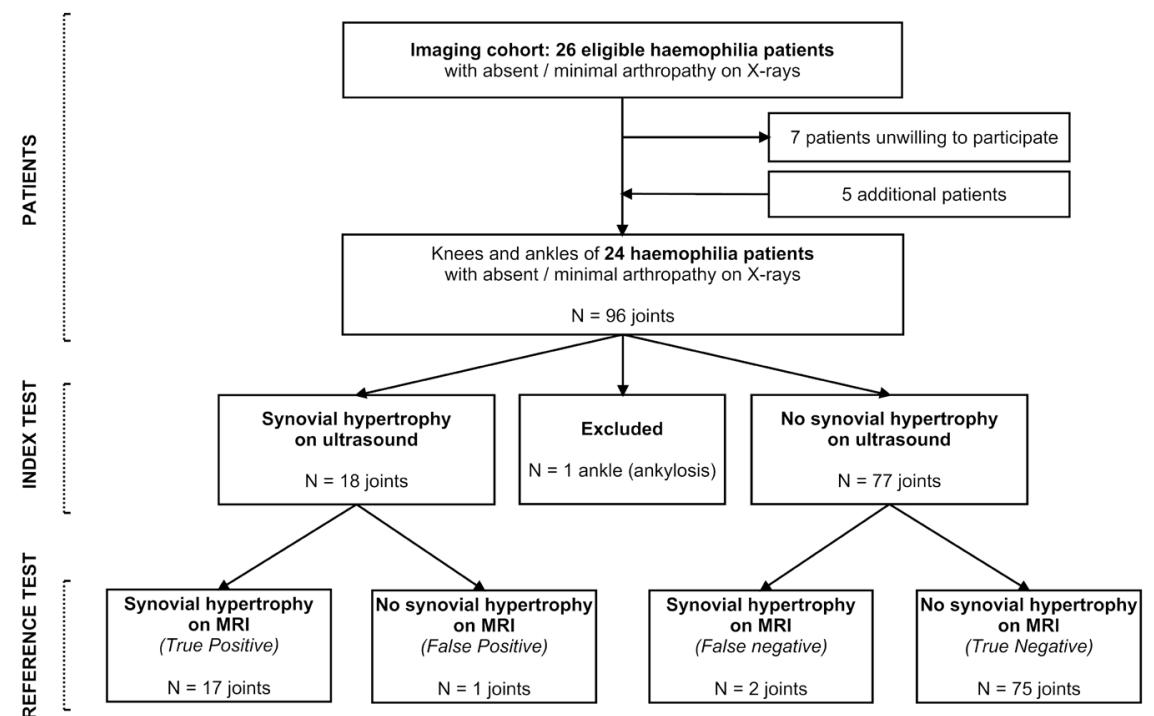

Figure 1. Flowchart

Baseline patient and joint characteristics are presented in Table 1. The median age of patients studied was 26.5 years (IQR: 23.0-29.5). In total, 17/24 patients $(71 \%)$ had severe haemophilia $\mathrm{A}$ and the majority of patients $(75 \%)$ received prophylaxis. Most joints (81\%) had a history of bleeding although minimal $\mathrm{X}$-ray changes were observed in 18\% of joints only (median Pettersson score: 0 , range: $0-6)$.

Table 1. Baseline patient and joint characteristics

\begin{tabular}{lccc}
\hline Patient characteristics & $\mathrm{n}=24$ patients & & \\
\hline Age in years (inter quartile range) & $26.5(23.0-29.5)$ & & \\
Severe haemophilia & $17(71 \%)$ & & \\
Prophylactic treatment & $18(75 \%)$ & & \\
Joint characteristics & Knees $(\mathrm{n}=48)$ & Ankles ( $=47)$ & Overall $(\mathrm{n}=95)$ \\
History of bleeding & $71 \%$ & $91 \%$ & $81 \%$ \\
X-rays abnormalities & $13 \%$ & $23 \%$ & $18 \%$ \\
MRI abnormalities & $69 \%$ & $68 \%$ & $68 \%$ \\
\hline
\end{tabular}

One ankle with ankylosis as excluded in the analyses 


\section{Test results}

POC-US and MRI were performed on the same day in all patients. No adverse events occurred during this study. Although MRI abnormalities were present in $68 \%$ of joints, minimal abnormalities were detected only: the median IPSG MRI score was 1 out of 17 points (IQR: $1-3)$. Synovial hypertrophy on MRI was observed in 3 knees $(6 \%)$ and in 16 ankles (34\%). For identification of synovial hypertrophy, POC-US was true positive in 17 joints, true negative in 75 joints, false positive in 1 joint, and false negative in 2 joints (Figure 1). Ultrasound and corresponding MRI findings of synovial hypertrophy, cartilage defects, and bone defects for knees and ankles are presented in Table 2.

Table 2. Results of point-of-care ultrasound and MRI

\begin{tabular}{|c|c|c|c|c|c|c|}
\hline & \multicolumn{2}{|c|}{ Knees $(n=48)$} & \multicolumn{2}{|c|}{ Ankles $(n=47)$} & \multicolumn{2}{|c|}{ Overall $(n=95)^{a}$} \\
\hline & MRI - & MRI + & MRI - & $\mathrm{MRI}+$ & MRI - & $\mathrm{MRI}+$ \\
\hline \multicolumn{7}{|c|}{ Synovial hypertrophy } \\
\hline POC-US - & 44 & 0 & 31 & 2 & 75 & 2 \\
\hline POC-US + & 1 & 3 & 0 & 14 & 1 & 17 \\
\hline \multicolumn{7}{|c|}{ Cartilage defects } \\
\hline POC-US - & 43 & 3 & 31 & 4 & 74 & 7 \\
\hline POC-US + & 0 & 2 & 2 & 10 & 2 & 12 \\
\hline \multicolumn{7}{|l|}{ Bone defects } \\
\hline POC-US - & 45 & 1 & 34 & 0 & 79 & 1 \\
\hline POC-US + & 0 & 2 & 2 & 11 & 2 & 13 \\
\hline
\end{tabular}

\section{Diagnostic accuracy estimates}

The presence/absence of synovial hypertrophy was correctly identified using POC-US in 94\% (CI: 86-100). For synovial hypertrophy POC-US had a sensitivity of $89 \%$ (CI: 67-99), specificity of 99\% (CI: 93-100), positive predictive value of $94 \%$ (CI: 73-100), and a negative predictive value of 97\% (CI: 91-100). The accuracy of POC-US for synovial hypertrophy was comparable in knees and ankles, except for a higher sensitivity $(100 \%$ in knees versus $88 \%$ in ankles) and lower positive predictive value in knees (75\% in knees versus 100\% in ankles) (Table 3). An example of synovial hypertrophy in the ankle is shown in Figure 2. 
The overall accuracy of POC-US for assessment of cartilage defects was $91 \%$ (CI: 83-96) and for bone defects 97\% (CI: 91-99). Negative predictive values for cartilage and bone defects were $>90 \%$ and significantly better than $70 \%$. Although the positive predictive values for cartilage and bone defects were $86 \%$ and $87 \%$ respectively, the wide confidence intervals still included the minimal acceptable threshold of $70 \%$ (Table 3).

Table 3. Diagnostic accuracy of point-of-care ultrasound

\begin{tabular}{lccccc}
\hline & Sensitivity & Specificity & Positive PV & Negative PV & Accuracy ${ }^{\text {a }}$ \\
\hline Synovial hypertrophy & & & & & \\
Knees & $100(37-100)$ & $98(88-100)$ & $75(19-99)$ & $100(93-100)$ & $98(89-100)$ \\
Ankles & $88(62-98)$ & $100(91-100)$ & $100(81-100)$ & $94(80-99)$ & $96(85-99)$ \\
$\quad$ Overall b & $89(67-99)$ & $99(93-100)$ & $94(73-100)$ & $97(91-100)$ & $97(91-99)$ \\
Cartilage defects & & & & & \\
Knees & $40(5-85)$ & $100(93-100)$ & $100(22-100)$ & $93(82-99)$ & $94(83-99)$ \\
Ankles & $71(42-92)$ & $94(80-99)$ & $83(52-98)$ & $89(73-97)$ & $87(74-95)$ \\
$\quad$ Overall b & $63(38-84)$ & $97(91-99)$ & $86(57-98)$ & $91(83-96)$ & $91(83-96)$ \\
Bone defects & & & & & \\
Knees & $67(9-99)$ & $100(94-100)$ & $100(22-100)$ & $98(88-100)$ & $98(89-100)$ \\
Ankles & $100(76-100)$ & $94(81-99)$ & $85(55-98)$ & $100(92-100)$ & $96(86-99)$ \\
Overall ${ }^{\text {b }}$ & $93(66-100)$ & $98(91-100)$ & $87(60-98)$ & $99(93-100)$ & $97(91-99)$ \\
\hline
\end{tabular}

Values are percentages ( $95 \%$ confidence intervals). PV, predictive value.

${ }^{a}$ Percentage correctly classified. ${ }^{b}$ Knees and ankles combined 

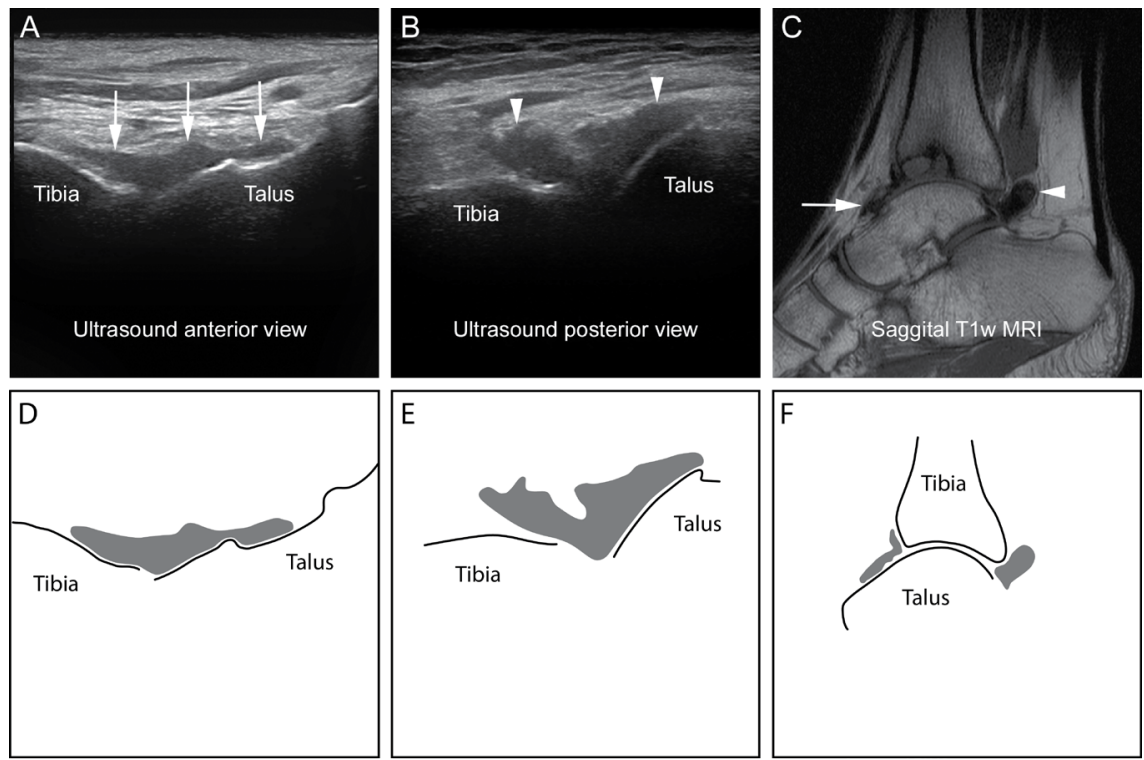

Figure 2. Synovial hypertrophy in the left ankle of a 25 -year-old haemophilia patient

A) Anterior longitudinal ultrasound image with synovial hypertrophy (arrows) covering the talar dome. B) Posterior view obtained medially from the Achilles tendon showing synovial hypertrophy (arrowheads). C) Corresponding sagittal T1-weighted MRI of the left ankle showing synovial hypertrophy with low signal haemosiderin deposits in the anterior (arrow) and posterior (arrowhead) joint recesses. Subchondral cysts in the distal tibia present as well. D-F) Schematic representation of synovial hypertrophy (grey) in figures A-C, respectively.

\section{Discussion}

This study shows that POC-US according to the HEAD-US protocol can accurately assess the presence/absence of synovial hypertrophy in joints of haemophilia patients. POC-US had an overall accuracy above $90 \%$ for the presence/absence of synovial hypertrophy, cartilage defects, and bone defects. For synovial hypertrophy, the positive and negative predictive values were $94 \%$ and $97 \%$ respectively and significantly higher than the minimal acceptable threshold of $70 \%$.

Joints were assessed using a standardized POC-US protocol and IPSG MRI score $[12,15]$. The operator dependency of ultrasound may be a disadvantage in clinical practice. The HEAD-US protocol has shown a good reliability when performed by non-radiologists with limited training only [17]. In the present study, ultrasound was performed by a single medical doctor who was trained with the HEAD-US protocol and had an experience of scanning over 
200 joints. A similar diagnostic accuracy of POC-US is expected if performed by observers with comparable training and experience.

The IPSG MRI score was developed and evaluated without the use of contrast agents [15]. As a result, subtle synovial hypertrophy may have been undetected in the present study as MRI without contrast has shown to be less sensitive for synovial hypertrophy in Juvenile Idiopathic Arthritis [18]. Consequently, the sensitivity and positive predictive value of POC-US of synovial may have been overestimated in our study. However, this is unlikely as synovial hypertrophy according to the IPSG MRI score is evaluated using robust surface measurements in a single MRI slice only [16,19]. Therefore, MRI with contrast is not expected to change the classification of synovial hypertrophy according to the haemophilia specific MRI score.

The diagnostic accuracy of POC-US for the presence/absence of cartilage and bone defects was assessed as secondary objective in this study. Although the positive and negative predictive values of POC-US for cartilage and bone defects were $85-99 \%$, the positive predictive values were not significantly better than $70 \%$ as the required sample size was calculated for the accuracy of POC-US for synovial hypertrophy only. However, POC-US may provide a general indication of cartilage and bone status in patients with no or limited abnormalities on X-rays.

\section{Comparison with other studies}

Although studies comparing ultrasound with MRI in haemophilia were performed previously [9,10], none of them used POC-US. Recently, Doria et al. reported the diagnostic accuracy of ultrasound in patient with haemophillia or von Willberand disease [10]. Ultrasound was performed and interpreted by sonographers and radiologists according to a comprehensive ultrasound protocol described by Zukotynski [20]. Observes were not blinded for MRI findings in order to study maximum achievable diagnostic accuracy. The overall prevalence of synovial hypertrophy was 93\% (compared to $20 \%$ in our study). Ultrasound was highly sensitive (100\%, CI: 95-100) in detecting synovial hypertrophy although the specificity of detection of synovial hypertrophy in ankle joints was 50\% only (CI: 7-93) (Table 4). Sierra Aisa et al. studied haemophilia patients, aged 4-82 years, using ultrasound and MRI [9]. The ultrasound protocol described by Zukotynski et al. [20] was used is their study too. The overall prevalence of synovial hypertrophy was $27 \%$ [9]. Diagnostic accuracy parameters were calculated from the results presented 
and are shown in Table 4. Even though the populations and study designs of previous studies differed from our study, ultrasound showed high positive and negative predictive values for the presence/absence of synovial hypertrophy. Although joints with no or minimal arthropathy were evaluated only, POCUS for synovial hypertrophy is not hampered by cartilage and minimal bone defects as the presence/absence is evaluated in the joint recesses only. The results on the diagnostic accuracy of POC-US for synovial hypertrophy are therefore expected to be valid for patient with moderate arthropathy too.

Table 4. Results of studies comparing ultrasound with MRI to detect synovial hypertrophy

\begin{tabular}{|c|c|c|c|}
\hline Study characteristics & Sierra Aisa et al. [9] & Doria et al. [10] & Present study \\
\hline Study population & $\begin{array}{l}\text { Haemophilia } \\
\text { (4-82yrs) }\end{array}$ & $\begin{array}{c}\text { haemophilia and VWD } \\
\text { (5-17yrs) }\end{array}$ & $\begin{array}{l}\text { Haemophilia } \\
\text { (19-35yrs) }\end{array}$ \\
\hline Joints & 60 knees, 60 ankles & 25 knees, 34 ankles & 48 knees, 47 ankles \\
\hline Ultrasound protocol & Zukotynski et al.[20] & Zukotynski et al. [20,21] & Martinoli et al. [12] \\
\hline Operators / observers & not reported & $\begin{array}{l}\text { sonographers \& } \\
\text { radiologists }\end{array}$ & medical doctor \\
\hline Blinding & not reported & unblinded for MRI & blinded for MRI \\
\hline \multicolumn{4}{|l|}{ Synovial hypertrophy } \\
\hline Knees & Prevalence $=12 \%$ & Prevalence $=92 \%$ & Prevalence $=6 \%$ \\
\hline Sensitivity & $86(42-100)$ & $100(85-100)$ & $100(37-100)$ \\
\hline Specificity & $96(80-100)$ & $100(16-100)$ & $98(88-100)$ \\
\hline Positive PV & $86(42-100)$ & $100(85-100)$ & 75 (19-99) \\
\hline Negative PV & $96(80-100)$ & $100(16-100)$ & $100(93-100)$ \\
\hline Ankles & Prevalence $=42 \%$ & Prevalence $=94 \%$ & Prevalence $=34 \%$ \\
\hline Sensitivity & $100(89-100)$ & $100(85-100)$ & $88(62-98)$ \\
\hline Specificity & $100(55-100)$ & $50(1-99)$ & $100(91-100)$ \\
\hline Positive PV & $100(89-100)$ & $97(84-100)$ & $100(81-100)$ \\
\hline Negative PV & $100(55-100)$ & $100(3-100)$ & $94(80-99)$ \\
\hline Overall $^{\text {a }}$ & Prevalence $=27 \%$ & Prevalence $=93 \%$ & Prevalence $=20 \%$ \\
\hline Sensitivity & $97(94-100)$ & $100(95-100)$ & $89(67-99)$ \\
\hline Specificity & $96(82-100)$ & $50(7-93)$ & $99(93-100)$ \\
\hline Positive PV & $97(94-100)$ & $96(88-100)$ & $94(73-100)$ \\
\hline Negative PV & $96(82-100)$ & $100(22-100)$ & $97(91-100)$ \\
\hline
\end{tabular}

Values are percentages (95\% confidence intervals). VWD, von Willebrand disease; PV, predictive value; VWD, von Willebrand disease. ${ }^{a}$ Knees and ankles combined 


\section{Clinical application}

POC-US is increasingly used in haemophilia patients to obtain direct information of joint status [22-25]. A recent report showed that synovial hypertrophy detected by MRI is predictive for joint bleeding in patients with limited arthropathy [7]. As POC-US can accurately evaluate the presence of synovial hypertrophy, it may be used to monitor treatment and/or to identify joints at risk of bleeding in these patients. Further studies using POC-US are needed to assess its diagnostic accuracy in other centres and to evaluate the minimal expertise required. It remains to be evaluated in what way POCUS detected synovial hypertrophy may guide treatment changes and if these treatment changes could improve joint outcome.

\section{Conclusion}

The positive and negative predictive value of POC-US for synovial hypertrophy were high at $94 \%$ and $97 \%$ respectively. As synovial hypertrophy is associated with bleeding, POC-US performed by trained clinicians may play a role in tailoring haemophilia treatment in the future. Further studies are needed to establish the effects of POC-US guided treatment changes. 


\section{References}

1 Iorio A, Marchesini E, Marcucci M, Stobart K, Chan A. Clotting factor concentrates given to prevent bleeding and bleeding-related complications in people with hemophilia A or B ( Review ). Cochrane Database Syst Rev 2011 1-46.

2 Srivastava A, Brewer AK, Mauser-Bunschoten EP, et al. Guidelines for the management of hemophilia. Haemophilia 2013; 19: e1-47.

3 Jansen NW, Roosendaal G, Lafeber FP. Understanding haemophilic arthropathy: an exploration of current open issues. Br J Haematol 2008; 143: 632-40.

4 Lafeber FPJG, Miossec P, Valentino L a. Physiopathology of haemophilic arthropathy. Haemophilia 2008; 14: 3-9.

5 Den Uijl IEM, De Schepper A, Camerlinck M, Grobbee DE, Fischer K. Magnetic resonance imaging in teenagers and young adults with limited haemophilic arthropathy: baseline results from a prospective study. Haemophilia 2011; 17: 926-30.

6 Pergantou H, Platokouki H, Matsinos G, et al. Assessment of the progression of haemophilic arthropathy in children. Haemophilia 2010; 16: 124-9.

7 Foppen W, Fischer K. Prognostic Value of MRI Synovial Changes in Haemophilia Patients: Preliminary Results. Haemophilia 2015; 21 (s2): 41-2.

8 Chan MW, Leckie A, Xavier F, et al. A systematic review of MR imaging as a tool for evaluating haemophilic arthropathy in children. Haemophilia 2013; 19: e324-34.

9 Sierra Aisa C, Lucía Cuesta JF, Rubio Martínez A, et al. Comparison of ultrasound and magnetic resonance imaging for diagnosis and follow-up of joint lesions in patients with haemophilia. Haemophilia 2014; 20: e51-7.

10 Doria AS, Keshava SN, Mohanta A, et al. Diagnostic Accuracy of Ultrasound for Assessment of Hemophilic Arthropathy: MRI Correlation. Am J Roentgenol 2015; 204: W336-47.

11 Moore CL, Copel JA. Point-of-Care Ultrasonography. N Engl J Med 2011; 364: 749-57.

12 Martinoli C, Della Casa Alberighi O, di Minno G, et al. Development and definition of a simplified scanning procedure and scoring method for Haemophilia Early Arthropathy Detection with Ultrasound (HEAD-US). Thromb Haemost 2013; 109: 1170-9.

13 Bossuyt PM, Reitsma JB, Bruns DE, et al. Towards complete and,accurate reporting of studies of diagnostic accuracy: the STARD initiative. Radiology 2003; 226: 24-8.

14 Pettersson H, Ahlberg A, Nilsson IM. A radiologic classification of hemophilic arthropathy. Clin Orthop Relat Res 1980; 149: 153-9.

15 Lundin B, Manco-Johnson ML, Ignas DM, et al. An MRI scale for assessment of haemophilic arthropathy from the International Prophylaxis Study Group. Haemophilia 2012; 18: 962-70.

16 Nuss R, Kilcoyne R. The MRI Atlas of Hemophilic Arthropathy. NewYork: Professional Publishing Group, Ltd; 2002.

17 Fischer K, Oldenburg J, Astermark J, et al. Ultrasound evaluation of haemophilic joints by haemophilia physicians: a reliablity study. J Thromb Haemost 2015; 13 (s2): 601.

18 Hemke R, Kuijpers TW, Van Den Berg JM, et al. The diagnostic accuracy of unenhanced MRI in the assessment of joint abnormalities in juvenile idiopathic arthritis. Eur Radiol 2013; 23: 1998-2004. 
Point-of-care ultrasound diagnostic accuracy compared to MRI

19 Lundin B, Berntorp E, Pettersson H, et al. Gadolinium Contrast Agent is of Limited Value for Magnetic Resonance Imaging Assessment of Synovial Hypertrophy in Hemophiliacs. Acta Radiol 2007; 48: 520-30.

20 Zukotynski K, Jarrin J, Babyn PS, et al. Sonography for assessment of haemophilic arthropathy in children: a systematic protocol. Haemophilia 2007; 13: 293-304.

21 Keshava SN, Gibikote S, Mohanta A, Doria a. S. Refinement of a sonographic protocol for assessment of haemophilic arthropathy. Haemophilia 2009; 15: 1168-71.

22 Ceponis A, Wong-Sefidan I, Glass CS, von Drygalski A. Rapid musculoskeletal ultrasound for painful episodes in adult haemophilia patients. Haemophilia 2013; 19: 790-8.

23 Foppen W, van der Schaaf IC, Fischer K. Value of routine ultrasound in detecting early joint changes in children with haemophilia using the "Haemophilia Early Arthropathy Detection with UltraSound" protocol. Haemophilia 2015 , doi: 10.1111/hae.12769. [Epub ahead of print].

24 Kidder W, Nguyen S, Larios J, et al. Point-of-care musculoskeletal ultrasound is critical for the diagnosis of hemarthroses, inflammation and soft tissue abnormalities in adult patients with painful haemophilic arthropathy. Haemophilia 2015; 21: 530-7.

25 Aznar JA, Pérez-Alenda S, Jaca M, et al. Home-delivered ultrasound monitoring for home treatment of haemarthrosis in haemophilia A. Haemophilia 2015 (in press). 


\section{CHAPTER 7}

\section{Value of point-of-care ultrasound in detecting early joint changes in children with haemophilia}

Published in Haemophilia (2016) Originally entitled: Value of routine ultrasound in detecting early joint changes in children with haemophilia using the 'Haemophilia Early Arthropathy Detection with UltraSound' protocol

W. Foppen ${ }^{1}$, I.C. van der Schaaf ${ }^{1}$, K. Fischer ${ }^{2,3}$

1 Department of Radiology; 2 Julius Center for Health Sciences and Primary Care; and 3 Van Creveldkliniek, Department of Hematology, University Medical Center Utrecht, Utrecht, The Netherlands. 


\section{Abstract}

\section{Introduction}

Patients with haemophilia tend to bleed in large joints even during prophylactic replacement therapy. Detection of early blood-induced joint changes may improve monitoring of treatment. The aim of this study was to explore the value of point-of-care ultrasound in detecting early joint abnormalities in children with haemophilia on prophylaxis.

\section{Methods}

Sixty-four joints in 32 children with haemophilia were examined by one operator using the Haemophilia Early Arthropathy Detection with UltraSound (HEAD-US) protocol during annual multidisciplinary follow-up. Based on reported bleeding, the joint with the highest risk of blood-induced joint damage and the contralateral joint were examined. At the same day, clinical function was assessed according to the Haemophilia Joint Health Score (HJHS).

\section{Results}

Median age was 11.5 years (range $=5.5-16.4$ ). Out of the 64 examined joints, one ankle was excluded because of previous surgery. Median lifetime joint bleeds $/$ joint was three (interquartile ranges $=1-5$ ). Clinical function of most joints was perfect: only $7 / 49$ joints with reported bleeds scored positive due to swelling, muscle atrophy and/or range of motion loss (HJHS range = 1-2 points). Ultrasound showed abnormalities in 5/49 joints with reported bleeding, and 4/5 showed positive HJHS scores. Ultrasound abnormalities were present in $1 / 56$ joints $(1.8 \%$, CI: $0.1-9.6 \%)$ without loss of clinical function.

\section{Conclusions}

Ultrasound abnormalities were found during routine evaluation of joints in children with haemophilia on prophylaxis. Most joints with ultrasound abnormalities showed low HJHS scores too. Point-of-care ultrasound could be used to evaluate whether minimal losses of clinical function might be caused by anatomical changes. 


\section{Introduction}

Patients with severe haemophilia tend to bleed in the large joints. Repeated joint bleeds eventually induce synovial hypertrophy and progressive osteochondral changes [1]. The elbows, knees and ankles are the most affected joints [2, 3]. The aim of prophylactic replacement therapy for patients with haemophilia is to prevent bleeding and subsequent damage.

Traditionally, monitoring of prophylaxis included bleeding history, and sometimes examination of trough coagulation factor levels and/or X-rays. However, bleeding is mostly self-reported and prone to misclassification [4]. The study by Manco-Johnson and colleagues [5] observed joint changes on magnetic resonance imaging (MRI) in the absence of reported bleeding in some children who did not receive prophylaxis. Lundin et al. [6] observed MRI changes in 38\% of ankles in children without reported joint bleeds. Maintaining minimum trough levels of Factor VIII (FVIII)/FIX of 1-2\% does not guarantee prevention of bleeding $[7,8]$. The possibility of subclinical joint bleeds makes it even more important to monitor treatment by examining joints for early changes in synovial tissue and cartilage.

Since X-rays mainly visualize osteochondral changes, this imaging technique is not sensitive enough to show early joint changes [2,9]. The most sensitive imaging modality is MRI, which is capable to detect synovial hypertrophy, haemosiderin deposits, early cartilage defects as well as bony changes [10, 11]. However, MRI is not ideal for routine monitoring of joint status due to restricted availability, rather long scanning times and possible need for sedation in young patients. Therefore, ultrasound is an option worth considering in this case. Ultrasound is able to detect the typical haemophilia-related joint changes such as synovial hypertrophy and cartilage defects [12-14]. Disadvantages of ultrasound are the operator dependency and long imaging protocols [14-16].

Martinoli and colleagues recently developed a point-of-care ultrasound protocol with associated score for non-radiologists [17]. After a short period of training, it is possible for physicians to examine joints of patient with haemophilia with ultrasound at the outpatient clinics for direct information on the joint status. So far, the effectiveness of this point-of-care ultrasound protocol in routine assessment for detection of early arthropathy has not been reported.

The aim of this study was to explore the value of point-of-care ultrasound for detection of early arthropathy in children with haemophilia on prophylaxis. 


\section{Materials and methods}

This retrospective cohort study included all children with moderate or severe haemophilia on prophylaxis who were examined with ultrasound for early arthropathy during annual multidisciplinary follow-up between September 2013 and August 2014. In total, 32 children without known joint damage were included. The study was approved by the Institutional Review Board of the University Medical Center Utrecht, The Netherlands.

Age, type of haemophilia, severity of disease, type of treatment and lifetime reported bleeding history were extracted from patient records. Based on the lifetime reported bleeding, the joint with the highest risk of arthropathy and its contralateral joint were assessed for side-to-side comparison. Joints were examined by a single medical doctor (WF) according to the Haemophilia Early Arthropathy Detection with UltraSound (HEAD-US) scanning protocol [17]. The presence and degree of synovial hypertrophy, cartilage defects and abnormalities of subchondral bone were scored. A single ultrasound scanner (type MyLab25 Gold; Esaote, Genova, Italy) with a linear probe (emission range $=5-13 \mathrm{MHz}$ with adjustable central frequency at 7.5, 10 and $12 \mathrm{MHz}$ ) was used for all subjects.

Clinical function was assessed on the same day by a physiotherapist according to the Haemophilia Joint Health Score (HJHS) version 2.1. The HJHS 2.1 evaluates for each joint potential swelling, muscle atrophy, crepitus on motion, flexion loss, extension loss, joint pain and loss of strength [18]. The HJHS on joint level ranges from 0 points, for joint with a perfect clinical function, up to 20 points for joints with severe loss of clinical function.

The order of assessment of clinical function and ultrasound examination alternated among patients. As the examinations were part of routine clinical care, findings of the physiotherapist regarding clinical function could be communicated by the patient or parents to the ultrasound operator. Consequently, the physiotherapist and the ultrasound operator were not blinded for each other's findings.

\section{Analysis}

Medians with interquartile ranges (IQR) or percentages with 95\% CI were calculated for patient and joint characteristics as well as ultrasound findings. Ultrasound changes were analysed according to lifetime reported bleeding and clinical function (HJHS). Spearman's correlation was used to analyse 
whether reported bleeding and clinical function (HJHS) were associated with ultrasound abnormalities (HEAD-US score) [17]. Correlations of $0.1-0.3$ were interpreted as weak, $0.3-0.5$ as moderate and $>0.5$ as strong [19].

\section{Results}

In total, six elbows, 20 knees and 38 ankles of 32 children with haemophilia were assessed. Median age of patients was 11.5 years (range $=5.5-16.4$ ). One ankle was excluded because of previous surgery. Overall, median number of lifetime joint bleeds per joint was three (IQR $=1-5)$ (Table 1).

Table 1. Patient and joint characteristics ( $\mathrm{n}=32$ patients, 63 joints)

\begin{tabular}{lcc}
\hline & median (IQR) or n $(\%)$ & range \\
\hline Age (years) & $11.5(9.5-13.1)$ & $5.5-16.4$ \\
Haemophilia A & $28(88 \%)$ & - \\
Severe haemophilia & $30(94 \%)$ & - \\
Prophylactic treatment & $32(100 \%)$ & \\
& & $0-11$ \\
Elbows (n $=3$ patients, 6 joints) & $3(0-4)$ & $0-2$ \\
Total bleeds & $0(0-1)$ & $0-2$ \\
Bleeds last year & $0(0-2)$ & $0-15$ \\
HJHS & & $0-2$ \\
Knees (n $=10$ patients, 20 joints) & $3(2-5)$ & $0-1$ \\
Total bleeds & $0(0-0)$ & \\
Bleeds last year & $0(0-0)$ & $0-14$ \\
HJHS & & $0-3$ \\
Ankles (n $=19$ patients, 37 joints) & $3(1-5)$ & $0-1$ \\
Total bleeds & $0(0-0)$ & $0(0-0)$ \\
Bleeds last year & & \\
HJHS
\end{tabular}

One ankle was excluded because of previous surgery

IQR, inter quartile ranges; HJHS, Haemophilia Joint Health Score

Ultrasound showed abnormalities in 5/63 joints only (7.9\%, CI: 2.6-17.6\%). Positive scores at physical examination and/or ultrasound abnormalities were observed in 8/49 joints with reported bleeds (16.3\%, CI: 7.3-29.7\%). These findings were only observed in patients with severe haemophilia A. None of the14 joints without reported bleeding (0\%, CI: 0-19.3\%) showed abnormalities at physical examination and/or ultrasound. Details on patient characteristics, reported joint bleeds, physical examination and ultrasound are shown in Table 2. 
Value of point-of-care ultrasound in detecting early joint changes

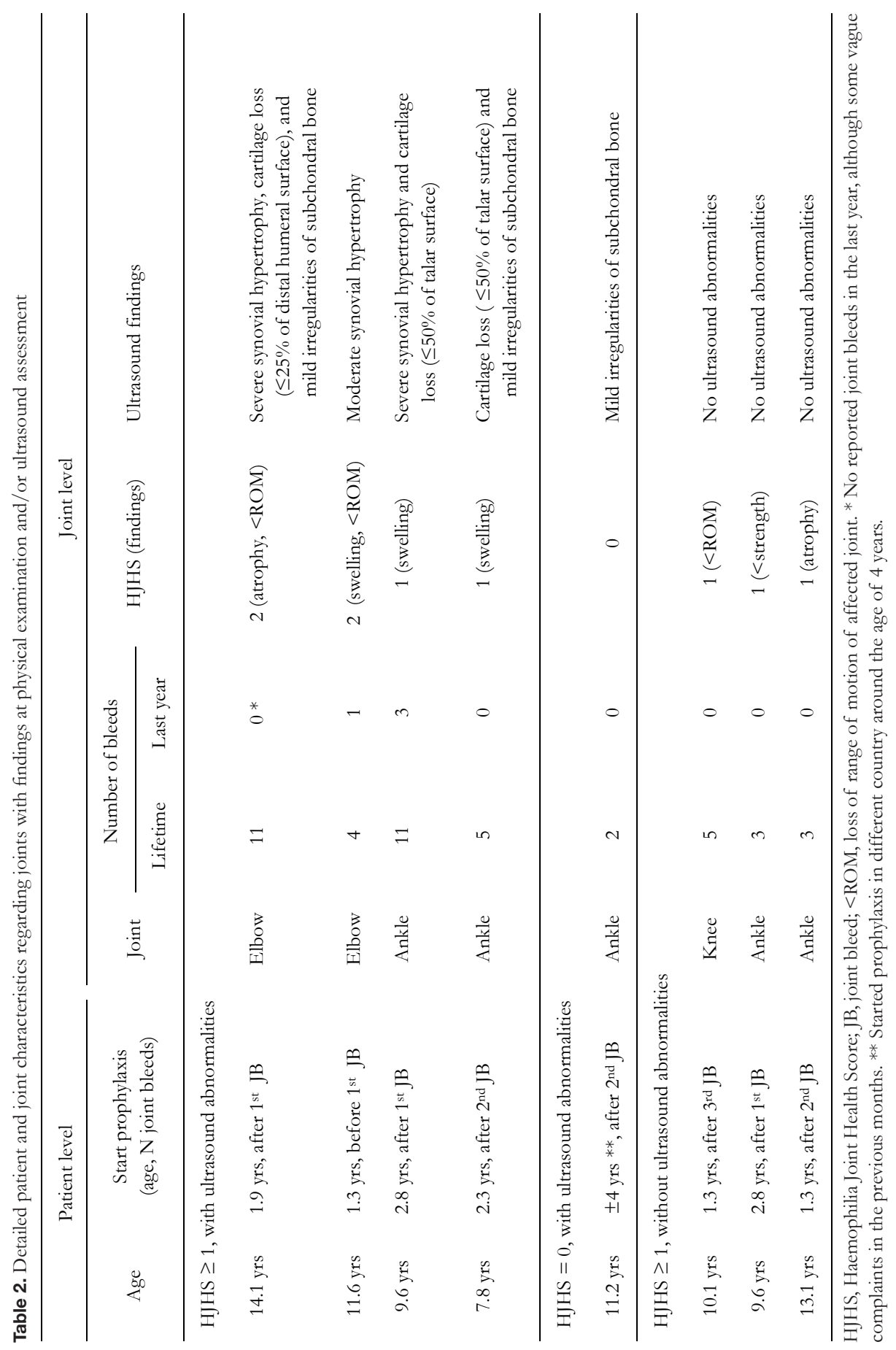




\section{Bleeding history and ultrasound findings}

In total, 49/63 joints (78\%) had reported bleeds, 33/63 joints (52\%) were exposed to $\geq 3$ bleeds in total, and $14 / 63$ joints (22\%) were exposed to $\geq 1$ bleed(s) in the last year before ultrasound examination. The observed ultrasound abnormalities were present in 5/49 joints (10.2\%, CI 3.4-22.2\%) with reported bleeds (Table 2, Figure 1). Joints without reported bleeding were all normal on ultrasound. The correlation between lifetime reported bleeds and HEAD-US score was moderate $(r=0.39, \mathrm{P}<0.01)$. Recent joint bleeds showed only a weak correlation $(r=0.25, \mathrm{P}=0.05)$ with ultrasound findings, abnormalities were found irrespective the presence or absence of a joint bleed in the last year.

\section{Clinical function and ultrasound findings}

Haemophilia Joint Health Score at joint level was 0 in $42 / 49$ joints $(86 \%)$ with reported bleeds, while the remaining $7 / 49$ joints $(14 \%)$ scored a median of one point (range $=1-2$ ) out of the maximum score of 20 points. None of the 14 joints without reported bleeding showed functional limitations. Overall, a strong correlation $(\mathrm{r}=0.70, \mathrm{P}<0.01)$ was observed between clinical function (HJHS) and ultrasound findings.

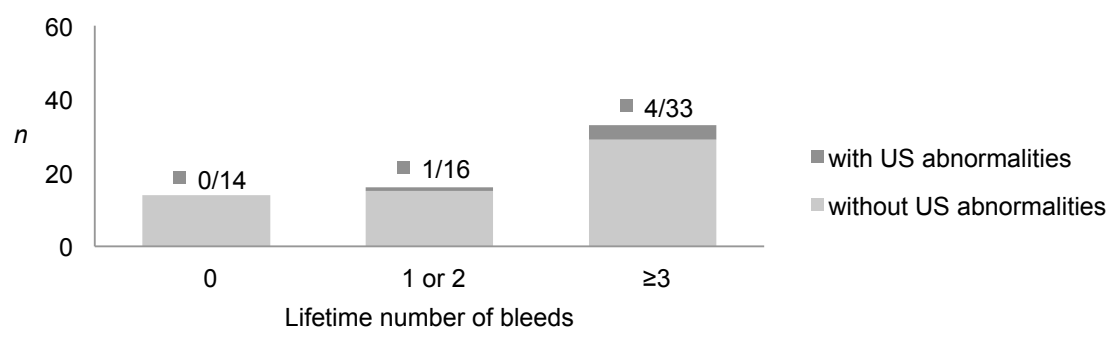

Figure 1. Ultrasound abnormalities according to lifetime reported bleeding

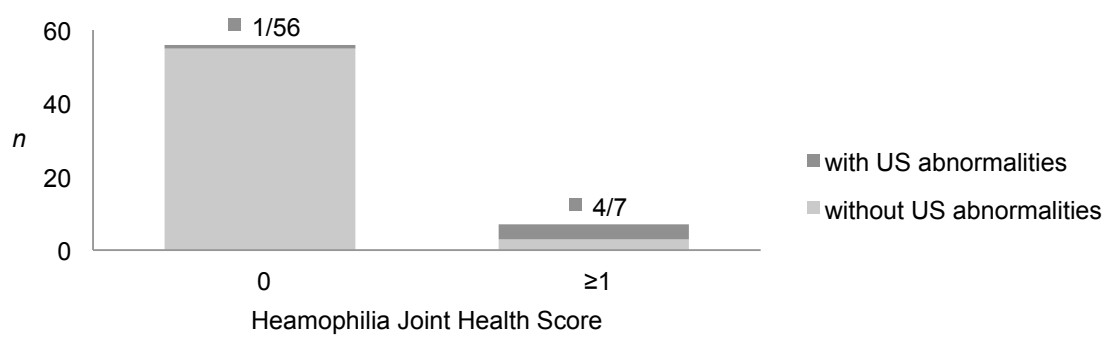

Figure 2. Ultrasound abnormalities according to Haemophilia Joint Health Scores 
Ultrasound changes were associated with loss of clinical function in the majority of cases as shown in Figure 2. The HJHS was positive in $4 / 5$ joints (80.0\%, CI: 28.4-99.5\%) with ultrasound abnormalities due to unilateral loss of extension, muscle atrophy and/or swelling (Table 2). In joints with loss of clinical function, ultrasound showed abnormalities in $4 / 7$ joints $(57.1 \%$, CI: 18.4-90.1\%). In these joints, ultrasound assessment showed synovial hypertrophy in $3 / 4$ joints, cartilage loss in $2 / 4$ joints and irregularities of subchondral bone in $2 / 4$ joints. By chance, clinical function was assessed before ultrasound examination in all joints with ultrasound abnormalities.

Discrepancies between results of clinical function and ultrasound assessment were found in $4 / 63$ joints $(6.4 \%, C I$ : $1.8-15.5 \%)$. Normal ultrasound with a slight loss of clinical function was observed in $3 / 63$ joints (4.8\%, CI: $0.1-$ $13.3 \%$ ). These joints scored positive on the HJHS due to unilateral loss of range of motion, loss of strength, or muscle atrophy (Table 2). Abnormal ultrasound findings without loss of clinical function were observed in 1/56 joints only $(1.8 \%$, CI: $0.1-9.6 \%)$. This case concerned an ankle joint with a HJHS score of zero, although ultrasound showed mild irregularities of subchondral bone.

\section{Discussion}

This study explored the value of point-of-care ultrasound in detecting early joint abnormalities in children with haemophilia by a new, relatively simple, and fast scanning protocol. In children with haemophilia on prophylaxis, ultrasound showed abnormalities in $5 / 49$ joints with reported bleeds $(10.2 \%)$. No ultrasound abnormalities were observed in joints without reported bleeding. Discrepancies between clinical function and ultrasound assessment were found in 4/63 joints only (6.3\%).

The results of the present study depend on the validity of the assessment tools used. Clinical function was assessed using the HJHS with established reliability and validity in children $[20,21]$. Although the reproducibility of the HEAD-US used by non-radiologists is currently under study (K. Fischer, pers. comm.), inter-observer differences are not an issue in the current study as all ultrasound examinations in the present study were performed by a single operator.

Usually, ultrasound examination is performed after taking patient's history and physical examination. As the evaluations were performed during regular outpatient clinics, the order of assessment of clinical function and ultrasound 
examination was not prespecified and varied among patients. As clinical function was assessed before the ultrasound examination in all five joints with ultrasound abnormalities, it is unlikely that the results of clinical function assessment are biased.

\section{Results of previous studies}

Results of physical examination and ultrasound assessment of joints were reported in previous studies too. Klukowska et al. [12] observed a strong correlation $(r=0.73)$ between lifetime cumulative number of joint bleeds and cartilage defects in children aged 2-16 years with many joint bleeds $>30$ joint bleeds in $64 \%$ of patients). Di Minno et al. [23] assessed clinical healthy joints (according to the World Federation of Haemophilia Orthopaedic Joint Score [22]) of 20 young adult patients (mean age $=22.5$ years, including $70 \%$ on prophylaxis). Synovial hypertrophy was observed in 55\% of joints, and cartilage defects in 80\% of joints [23]. Sierra Aisa et al. [24] found ultrasound abnormalities in about $30 \%$ of knees and in $50 \%$ of ankles in haemophilia patients (aged 4-82 years, 64\% treated on demand), but did not correlate these with clinical findings.

The reported incidence of ultrasound abnormalities in joints without clinical signs in these three studies is higher than the $1.8 \%$ observed in the present study. This discrepancy may be well explained by the fact that patients in the present study were younger and treated with early prophylaxis. In addition, clinical joint status in previous studies was assessed by a less sensitive assessment method resulting in the underestimation of the proportion of clinically affected joints; it is expected that some joints would have shown positive HJHS scores [18].

\section{Clinical relevance - physicians perspective}

In the present study, point-of-care ultrasound showed abnormalities in joints of children with haemophilia on prophylaxis. A slight loss of clinical function was observed in $4 / 5$ of these joints $(80.0 \%)$. In contrast, discrepancies between results of clinical function and ultrasound assessment were observed in $4 / 63$ joints $(6.4 \%)$ in total. This might be explained by the fact that some HJHS-items - such as pain, range of motion and loss of strength - cannot be evaluated with ultrasound. In addition, positive HJHS scores without confirmation on MRI have been described in healthy controls, aged 19-26 years. In these subjects, $15 / 120(12.5 \%)$ of knees and ankles had a HJHS of 1-3 points (crepitus, reduced range of motion) without changes on MRI [25, 26]. Thus, low scores on the HJHS do not necessarily reflect intra-articular abnormalities. 
What does detecting early joint abnormalities add in clinical management? Minimum levels of FVIII/IX of $1-2 \%$ do not prevent all joint bleeding [7, 8]. Despite initiating prophylaxis at a young age in the present study, $8 / 49$ joints $(16.3 \%)$ with reported bleeds had findings at physical examination and/or ultrasound assessment (Table 2). All these joints were exposed to bleeds after initiating prophylaxis, except one ankle with bleeding before prophylaxis only (HJHS $=0$, with ultrasound abnormalities). In case of abnormalities in clinical function, point-of-care ultrasound can be used to evaluate whether these findings are associated with intra-articular abnormalities.

The incidence and risks of subclinical intra-articular joint bleeding leading to joint changes are not well established. In the present study, joints without reported bleeding did not show abnormalities at physical examination (HJHS $\geq 1$ ) and/or ultrasound assessment. Unexpected ultrasound findings were observed in only $1 / 56$ joints $(1.8 \%)$ without clinical signs on HJHS. This might be explained by the examined young age and prophylactic treatment of patients studied. It is likely that unexpected imaging findings are more frequent in older patients, and/or when using a more sensitive imaging tool such as MRI [27].

\section{Clinical relevance - patients perspective}

Ultrasound findings are more tangible than minimal changes on physical examination. Since early arthropathy might go unnoticed by haemophilic children and their parents, visualization of early anatomical joint changes may be used to make treatment decisions and/ or promote adherence to prophylaxis. At the time of the patients next visit, joint status could be assessed with ultrasound to show the results of treatment since last evaluation. For clinical interpretation of ultrasound results, it is important to know the diagnostic accuracy of the HEAD-US protocol. This will have to be established by future studies.

\section{Conclusion}

Point-of-care ultrasound assessment of joints in children with moderate or severe haemophilia on prophylaxis showed early joint abnormalities, mostly in joints with low HJHS scores. The ability of ultrasound to visualize anatomical joint abnormalities could be used for follow-up of abnormalities and to evaluate whether minimal losses of clinical function might be caused by anatomical changes. 


\section{References}

1 Jansen NW, Roosendaal G, Lafeber FP. Understanding haemophilic arthropathy: an exploration of current open issues. Br J Haematol 2008; 143: 632-40.

2 Arnold WD, Hilgartner MW. Hemophilic arthropathy. Current concepts of pathogenesis and management. J Bone Jt Surg Am 1977; 59: 287-305.

3 Aronstam A, Rainsford SG, Painter MJ. Patterns of bleeding in adolescents with severe haemophilia A. Br Med J 1979; 1: 469-70.

4 Ceponis A, Wong-Sefidan I, Glass CS, von Drygalski A. Rapid musculoskeletal ultrasound for painful episodes in adult haemophilia patients. Haemophilia 2013; 19: 790-8.

5 Manco-Johnson MJ. Prophylaxis versus episodic treatment to prevent joint disease in boys with severe hemophilia. N Engl J Med 2007; 2007: 535-44.

6 Lundin B, Ljung R, Pettersson H. MRI scores of ankle joints in children with haemophilia-comparison with clinical data. Haemophilia 2005; 11: 116-22.

7 Den Uijl I, Biesma D, Grobbee D, Fischer K. Turning severe into moderate haemophilia by prophylaxis: are we reaching our goal? Blood Transfus 2013; 11: 364-9.

8 Ahnström J, Berntorp E, Lindvall K, Björkman S. A 6-year follow-up of dosing, coagulation factor levels and bleedings in relation to joint status in the prophylactic treatment of haemophilia. Haemophilia 2004; 10: 689-97.

9 Pettersson H, Ahlberg A, Nilsson IM. A radiologic classification of hemophilic arthropathy. Clin Orthop Relat Res 1980; 149: 153-9.

10 Lundin B, Manco-Johnson ML, Ignas DM, et al. An MRI scale for assessment of haemophilic arthropathy from the International Prophylaxis Study Group. Haemophilia 2012; 18: 962-70.

11 Chan MW, Leckie A, Xavier F, et al. A systematic review of MR imaging as a tool for evaluating haemophilic arthropathy in children. Haemophilia 2013; 19: e324-34.

12 Klukowska a, Czyrny Z, Laguna P, et al. Correlation between clinical, radiological and ultrasonographical image of knee joints in children with haemophilia. Haemophilia 2001; 7: 286-92.

13 Aznar JA, Abad-Franch L, Perez-Alenda S, et al. Ultrasonography in the monitoring of management of haemarthrosis. Haemophilia 2011; 17: 826-8.

14 Querol F, Rodriguez-Merchan EC. The role of ultrasonography in the diagnosis of the musculoskeletal problems of haemophilia. Haemophilia 2012; 18: e215-26.

15 Zukotynski K, Jarrin J, Babyn PS, et al. Sonography for assessment of haemophilic arthropathy in children: a systematic protocol. Haemophilia 2007; 13: 293-304.

16 Melchiorre D, Linari S, Innocenti M, et al. Ultrasound detects joint damage and bleeding in haemophilic arthropathy: a proposal of a score. Haemophilia 2011; 17: 112-7.

17 Martinoli C, Della Casa Alberighi O, di Minno G, et al. Development and definition of a simplified scanning procedure and scoring method for Haemophilia Early Arthropathy Detection with Ultrasound (HEAD-US). Thromb Haemost 2013; 109: 1170-9.

18 Feldman BM, Funk S, Hilliard P, Van Der Net J, Zourikian N, Berstrom B-M, Engelbert RHH, Abad A, Petrini P, Manco-Johnson M IPSG. Hemophilia Joint Health Score (HJSH) 2.12011.

19 Cohen J. Statistical Power Analysis for the Behavioural Sciences. 2nd ed. Hillsdale, NJ: Lawrence Erlbaum Associates; 1988. 
Value of point-of-care ultrasound in detecting early joint changes

20 Hilliard P, Funk S, Zourikian N, et al. Hemophilia joint health score reliability study. Haemophilia 2006; 12: 518-25.

21 Feldman BM, Funk SM, Bergstrom BM, et al. Validation of a new pediatric joint scoring system from the International Hemophilia Prophylaxis Study Group: validity of the hemophilia joint health score. Arthritis Care Res 2011; 63: 223-30.

22 Gilbert MS. Prophylaxis: musculoskeletal evaluation. Semin Hematol 1993; 30: 3-6.

23 Di Minno MND, Iervolino S, Soscia E, et al. Magnetic resonance imaging and ultrasound evaluation of "healthy" joints in young subjects with severe haemophilia A. Haemophilia 2013; 19: e167-73.

24 Sierra Aisa C, Lucía Cuesta JF, Rubio Martínez A, et al. Comparison of ultrasound and magnetic resonance imaging for diagnosis and follow-up of joint lesions in patients with haemophilia. Haemophilia 2014; 20: e51-7.

25 Foppen W, Sluiter D, Witkamp TD, Mali WPTM, Fischer K. Haemophilic magnetic resonance imaging score in healthy controls playing sports. Haemophilia 2013; 19: 939-43.

26 Sluiter D, Foppen W, de Kleijn P, Fischer K. Haemophilia Joint Health Score in healthy adults playing sports. Haemophilia 2014; 20: 282-6.

27 Den Uijl IEM, De Schepper A, Camerlinck M, Grobbee DE, Fischer K. Magnetic resonance imaging in teenagers and young adults with limited haemophilic arthropathy: baseline results from a prospective study. Haemophilia 2011; 17: 926-30. 



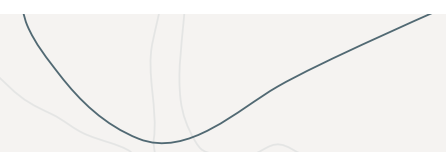

\section{General discussion and Summary}

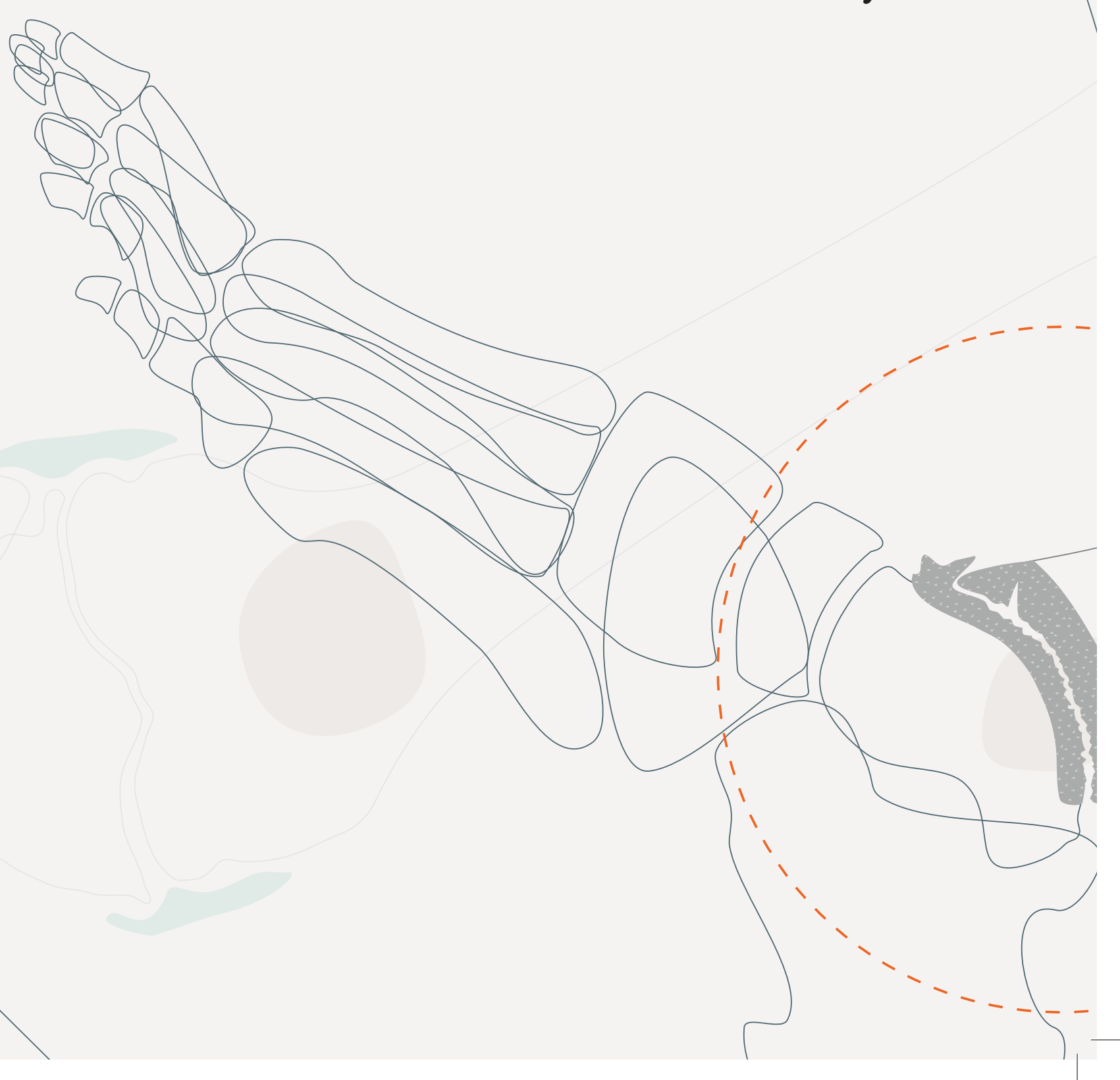




\section{CHAPTER 8}

\section{General discussion}




\section{Key findings in this thesis}

\section{X-rays}

- Using an atlas for scoring haemophilic arthropathy on X-rays improves reproducibility

\section{Magnetic resonance imaging}

- Sports participation is not associated with haemophilia specific joint changes in healthy adults

- Joint effusion on MRI is not a haemophilia specific finding

- Synovial hypertrophy predicts joint bleeding and haemophilia specific MRI findings predict progression of haemophilic arthropathy

\section{Point-of-care ultrasound (POC-US)}

- POC-US can accurately assess synovial hypertrophy

- POC-US can evaluate whether minimal loss of clinical function is associated with arthropathy

Haemophilia is characterised by joint bleeding caused by lack of coagulation factor VIII or IX activity. Recurrent or prolonged joint bleeding eventually leads to synovial hypertrophy and progressive osteochondral damage through mechanical and metabolic joint destruction [1,2]. The elbows, knees, and ankles are the most frequently affected joints [3-5]. Although coagulation factor replacement therapy (prophylaxis) for haemophilia is available, its high costs (US\$ 180,000 - 298,000 annually/patient [6]) hamper the use of coagulation factor substitution to normal levels to prevent all bleeding episodes. Patients treated with intermediate dose prophylaxis still report approximately two joint bleeds annually [7]. To optimise treatment, evaluation of the effects of prophylaxis in individual patients and among patient groups is needed.

\section{X-rays}

As prophylaxis according to the regimen used in the 1980s and 1990s did not fully prevent bleeding and development of arthropathy [8], moderate-severe haemophilic arthropathy is still common in haemophilia patients aged $>30$ years [9]. X-rays are the first choice to assess the bony changes in moderatesevere arthropathy, as this modality is widely available at low costs (Table 1). 
Table 1. Characteristics of imaging modalities for haemophilic arthropathy

\begin{tabular}{|c|c|c|c|c|}
\hline General aspects & $\mathrm{X}$-rays & MRI & POC-US & Comments \\
\hline Availability & + & - & + & \\
\hline Costs & + & - & + & $\begin{array}{l}\text { X-rays: } € 39 \text {. MRI: } € 239 \text {. POC-US: unknown. } \\
\text { Note: Costs for the lower extremity in the } \\
\text { Netherlands for } 2016 \text { [45]. }\end{array}$ \\
\hline Duration (time) & + & - & + & $\begin{array}{l}\text { X-rays: about } 2-5 \mathrm{~min} / \text { joint. MRI: about } 30 \mathrm{~min} / \\
\text { joint in clinical setting. US: about } 5 \mathrm{~min} / \text { joint. }\end{array}$ \\
\hline Diagnostic accuracy & $\mathrm{X}$-rays & MRI & POC-US & Comments \\
\hline $\begin{array}{l}\text { Effusion and/or } \\
\text { haemarthrosis }\end{array}$ & - & + & + & $\begin{array}{l}\text { POC-US: not scored with HEAD-US protocol } \\
\text { [38]. Note: Differentiation between effusion and } \\
\text { haemarthrosis is troublesome. }\end{array}$ \\
\hline Synovial hypertrophy & - & + & + & $\begin{array}{l}\text { MRI: MRI with contrast results in the best } \\
\text { identification [32]. POC-US: sensitivity } 89 \% \text {, } \\
\text { specificity } 99 \% \text { [chapter 6]. }\end{array}$ \\
\hline Haemosiderin & - & + & NA & $\begin{array}{l}\text { POC-US: No consensus whether haemosiderin } \\
\text { can be visualized with US [35,36,38]. Not } \\
\text { assessed with HEAD-US protocol [38]. }\end{array}$ \\
\hline Cartilage defects & \pm & + & \pm & $\begin{array}{l}\text { X-rays: Late changes only by a reduced joint } \\
\text { space. POC-US: sensitivity } 63 \% \text {, specificity } 97 \% \\
\text { [chapter 6]; evaluation of parts of joint only. }\end{array}$ \\
\hline Bone defects & \pm & + & \pm & $\begin{array}{l}\text { X-rays: Late changes only. POC-US: sensitivity } \\
93 \% \text {, specificity } 98 \% \text { [chapter 6]; evaluation of } \\
\text { parts of joint only. }\end{array}$ \\
\hline Outcome requirements & $\mathrm{X}$-rays & MRI & POC-US & Comments \\
\hline $\begin{array}{l}\text { Standardization of } \\
\text { imaging technique and } \\
\text { scoring }\end{array}$ & \pm & - & - & $\begin{array}{l}\text { X-rays: No standardization of imaging though } \\
\text { impact is likely low. Pettersson score adopted } \\
\text { by WFH [11]. MRI: No global standardization } \\
\text { of required imaging sequences. Single score } \\
\text { proposed [26]. US: Several described ultrasound } \\
\text { protocols with scores [38,46-49]. }\end{array}$ \\
\hline $\begin{array}{l}\text { Available reference atlas } \\
\text { for scoring }\end{array}$ & + & \pm & - & $\begin{array}{l}\text { X-rays: Published atlas for the Pettersson score } \\
\text { [16]. MRI: Atlas with examples, suboptimal } \\
\text { standardization and images [31]. US: Reference } \\
\text { images for HEAD-US protocol provided during } \\
\text { training sessions though not published. }\end{array}$ \\
\hline $\begin{array}{l}\text { Reproducibility of } \\
\text { scoring method }\end{array}$ & + & + & \pm & $\begin{array}{l}\text { X-rays: Recently re-studied and improved } \\
\text { reliability and agreement [16]. MRI: Established } \\
\text { reliability for MRI scores [26,50], tested among } \\
\text { developers of score only. Agreement not } \\
\text { evaluated. US: Established reliability for HEAD- } \\
\text { US protocol among developers of score [38] and } \\
\text { among haemophilia physicians [44]. }\end{array}$ \\
\hline
\end{tabular}

MRI, Magnetic Resonance Imaging; US, ultrasound; WFH, World Federation of Haemophilia. Legend: - = unsatisfactory; $\pm=$ moderate; $+=$ satisfactory-good. Note: MRI is considered as the best available non-invasive reference standard and therefore rated as ' + ' 
Since the 1980s, two scoring systems using X-rays have been available for classification of haemophilic arthropathy: the progressive Arnold-Hilgartner score and the additive Pettersson score $[3,10]$. The Pettersson score-adopted by the World Federation of Haemophilia [11] - has been used in comparative studies showing a favourable long-term radiological outcome for prophylactic treatment strategies [12,13]. In addition, early onset of prophylaxis and continuation into adulthood have been associated with favourable Pettersson scores $[9,14,15]$.

\section{How to classify haemophilic arthropathy on X-rays?}

It is unknown to what extent differences in scores are attributable to observervariation. The reliability and agreement of the Pettersson score was studied in this thesis and a consensus atlas with reference images for scoring was developed. Before development of the consensus atlas, scoring variation in arthropathy was $\leq 3$ points at joint level (maximum score 13) and $\leq 13$ points at patient level (maximum score 78). With use of the developed consensus atlas the observers scored more consistent: scoring variation was $\leq 2$ points at joint level and $\leq 6$ points at patient level [16]. As the scoring variation among observers is low with use of the consensus atlas, its use may allow scoring X-rays by multiple observers without hampering the comparison of Pettersson scores.

\section{X-rays: Limitations and future perspectives}

The consensus atlas was developed in order to improve the reproducibility of the Pettersson score. Its external validity still needs to be established, preferably in an independent study evaluating the observer-variation with the consensus atlas when used by others .

Compared to standard X-rays, computed tomography (CT) provides more detail and allows for evaluation in cross-sectional planes. Especially in paediatric patients, the radiation dose associated with CT is a drawback. Although the effective radiation dose for CT scans of the extremities is low, further reduction may be achieved by new image-reconstruction techniques: Iterative reconstruction techniques for CT may lower the radiation dose with $23 \%-76 \%$ and still provide a comparable image quality compared to the currently used filtered back-projection $[17,18]$. A radiation dose reduction of $50 \%$ would result in a dosages comparable to the dose of $\mathrm{X}$-rays in two directions [19]. Despite the low radiation dose of CT of the extremities, CT does not provide as much detailed information regarding soft tissue as MRI or ultrasound. 


\section{Magnetic resonance imaging}

The use of early prophylactic clotting factor replacement therapy has led to an enormous improvement of joint outcome $[8,13,20]$. This improvement, together with the delay of visible X-ray abnormalities, has led to an increased interest in detection of the first stages of arthropathy. MRI is especially useful for evaluation of synovial hypertrophy and cartilage defects caused by joint bleeding [21]. Several haemophilia specific MRI scoring systems have been developed [22-26]. The most recent MRI score was developed by the International Prophylaxis Study Group (IPSG); it includes soft tissue items (effusion / haemarthrosis, synovial hypertrophy, and haemosiderin) as well as osteochondral items (cartilage defects, surface erosions, and subchondral cysts) [26].

\section{Interpretation of MRI findings}

To be able to distinguish between sports related and haemophilia related MRI changes, a cohort of young active men without haemophilia was evaluated by MRI. Results suggested that sports participation is not associated with haemophilia specific MRI changes [27]. A comparison of joint effusion between haemophilia patients and young active healthy controls showed increased effusion in knees of controls and comparable effusion in ankles. The amount of effusion increased with more frequent sports participation. Therefore, joint effusion on MRI is unlikely to be haemophilia specific. Accordingly, it was suggested to exclude joint effusion from MRI scores as it may reduce the specificity for haemophilic arthropathy [28].

\section{Clinical application of MRI}

So far, the prognostic value of MRI findings was based on assumptions only. In this thesis it was shown that bleeding risk is significantly increased in joints with synovial hypertrophy. In addition, IPSG MRI findings in joints without changes X-rays (Pettersson score $=0$ ) are associated with progression of arthropathy five years later [29]. Therefore, it may be beneficial to guide treatment based on MRI findings. For example: In patients on prophylaxis, identification of synovial hypertrophy may indicate suboptimal treatment. Subsequently, the clinician may emphasize patients to adhere to prophylaxis, prophylaxis may be intensified, and/or anti-inflammatory medication may be considered. Or in patients considering to discontinue prophylaxis [9], detection of synovial hypertrophy would be a contraindication for discontinuation of treatment. Further studies are required to establish which MRI findings are reversible and if MRI findings are responsive to treatment changes. 
In patients with advanced arthropathy it may be difficult to differentiate between an acute bleed or a flare-up of arthropathy [30]. In case of a painful joint, most patients start self-administration of clotting factors (home-treatment) to stop suspected bleeding. A correct diagnosis is essential as treatment of a bleed with coagulation factors is costly. In theory, MRI assessment could be used to differentiate between bleeding (haemorrhagic effusion) and flareups of arthropathy (normal effusion). However, differentiation between normal effusion and haemarthrosis, in absence of fluid-fluid levels indicating haemathrosis, is difficult as MRI signals are relative to the surrounding tissues. For development of a set of diagnostic criteria to differentiate between an acute bleed or a flare-up of arthropathy, MRI is the most likely non-invasive reference standard. A dedicated MRI protocol to differentiate between effusion and minimal joint bleeding is currently under development.

\section{MRI: Limitations and future perspectives}

In our MRI studies, the prevalence of synovial hypertrophy might have been underestimated for two reasons. First, to avoid problems in the distinction between no and mild synovial hypertrophy, we defined a minimal thresholds for synovial hypertrophy for our studies. As neither the IPSG MRI score nor the MRI atlas for haemophilic arthropathy state a minimal threshold for scoring synovial hypertrophy [26,31], we based our definitions on reference images in the MRI atlas for haemophilic arthropathy [31]. E.g. the minimal threshold for mild synovial hypertrophy was defined as an area of $\geq 0.50 \mathrm{~cm}^{2}$ for knees, measured in one slice in the sagittal view (chapter 5 and 6$)$. Therefore any subtle synovial hypertrophy $\left(<0.50 \mathrm{~cm}^{2}\right.$ in knees) would consequently be downgraded to no synovial hypertrophy. Second, we performed MRI scans without intravenous contrast, which may have reduced the ability to detect minimal synovial hypertrophy [32]. Although minimal synovial hypertrophy may be detected using contrast enhanced MRI, the atlas dictates that synovial hypertrophy in haemophilia is scored using robust surface measurements on a single MRI slice only [31]. Therefore, the value of contrast enhanced MRI is limited for scoring synovial hypertrophy in haemophilia patients [33]. Identification of minimal synovial hypertrophy on contrast enhanced MRI would not result in a shift towards another category as its surface is expected to be below our minimal threshold for synovial hypertrophy. As synovial hypertrophy on MRI without contrast predicted the joint bleeding risk very well, the additional value of contrast enhanced MRI in haemophilic arthropathy patients is expected to be limited. 
Studies comparing MRI outcomes are hindered by use of different imaging protocols and the lack of an up-to-date reference atlas for the (IPSG) MRI score. As external validation of the IPSG MRI score has not been performed yet, interpretation and scores may vary among radiologists. Standardization of MRI protocols for haemophilic arthropathy as well as assessment of the reproducibility of scoring would facilitate international comparison of data by avoiding the need for central scoring.

\section{Point-of-care ultrasound}

Ultrasound enables evaluation of fluid collections, synovial hypertrophy, and some cartilage and bone surfaces (Table 1) [34-36]. When performed by the treating physician, ultrasound may provide a clinically practicable alternative for MRI in joint evaluation in daily practice. Across different medical specialties, non-radiologists increasingly use point-of-care ultrasound (POC-US) for procedural, diagnostic, and screening purposes [37]. Recently, the 'Haemophilia Early Arthropathy Detection with Ultrasound' (HEADUS) protocol has been developed as a point-of-care ultrasound (POC-US) procedure for haemophilia clinicians. This protocol requires approximately 5 minutes per joint only [38]. POC-US at the outpatient clinic may be used to provide a valuable addition to history and physical examination and to guide treatment.

\section{Value of point-of-care ultrasound}

We established that in young adults with limited arthropathy, POC-US provides an accurate alternative for MRI for the assessment of synovial hypertrophy. For evaluation of synovial tissue, POC-US had a positive predictive value of 94\% (CI: 73-100), a negative predictive value of 97\% (CI: 91-100), and an overall accuracy of 97\% (CI: 91-99). In addition to identification of synovial hypertrophy, POC-US may provide a general indication of cartilage and bone status in patients with no or limited abnormalities on X-rays. The overall accuracy of POC-US was 91\% (CI: 83-96) for cartilage defects and 97\% (CI: 91-99) for bone defects. For evaluation of synovial hypertrophy, POCUS is not hampered by minimal bone defects as the presence/absence of synovial changes is evaluated in the joint recesses only. Therefore, our findings regarding the diagnostic accuracy of POC-US for synovial hypertrophy in patients with limited arthropathy are expected to be valid for patients with moderate arthropathy too. 
Recently, we explored the value of POC-US ultrasound for evaluation of early joint abnormalities in 32 children with severe haemophilia. Unexpected POC-US abnormalites were observed in only $2 \%$ of joints with a normal clinical function. In contrast, $57 \%$ of joints with a limited loss of clinical function showed abnormalities at POC-US evaluation. These results suggest that POC-US may be used in children to evaluate whether minimal losses of clinical function can be attributed to anatomical changes [39].

\section{Point-of-care ultrasound: Limitations and future perspectives}

Power Doppler ultrasound may be used to assess vascular changes in the haemophilic synovial tissue, as it correlates well $(\mathrm{r}=0.73, \mathrm{P}<0.01)$ compared to contrast enhanced MRI [40]. However, the use of Power Doppler in POC-US is still under debate. Based on the argument that vascular changes in synovial hypertrophy in haemophilia are mild and may represent normal vasculature, it was excluded from the HEAD-US protocol [38]. In contrast, other publications have suggested that Power Doppler ultrasound in haemophilia could predict increased bleeding risk $[41,42]$.

Recommendations for training, competency evaluation, and the scope of practice for POC-US in haemophilia were published recently [43]. The HEAD-US protocol has shown good reliability when performed by nonradiologists with the basic training only [44]. Further studies using POC-US are needed to assess the diagnostic accuracy in other centres and to evaluate the minimal expertise required.

\section{Clinical application}

How to use imaging in haemophilia care and research? For both clinical care and research, the optimal imaging modality depends on the clinical joint status. X-rays are mostly indicated for moderate-severe arthropathy as they are insensitive to early joint changes (Table 1). In order to provide an anatomical overview, X-rays may be used for follow-up of arthropathy and for surgical planning in patients with end-stage arthropathy. MRI is the most sensitive imaging modality for the assessment of initial joint changes such as synovial hypertrophy, haemosiderin, and cartilage defects. Its use is therefore recommended for assessment of the progression of arthropathy, or to evaluate the origin of clinical complaints. As synovial hypertrophy on MRI is associated with an increased joint bleeding risk, treatment may be monitored or tailored based on the presence/absence of synovial hypertrophy on imaging. 
Although the availability of MRI is limited, POC-US provides an accurate alternative for routine assessment of synovial hypertrophy. Further studies are required to establish the effects of imaging-guided treatment decisions. Both MRI and POC-US may potentially be useful for differentiation between normal- and haemorrhagic effusion. A dedicated MRI protocol is currently under development and preliminary results suggest that MRI is able to identify small amounts of blood in synovial fluid. Therefore, MRI may be used in the future to establish joint bleeding and avoid the need for joint aspiration. After confirmation of findings, the next steps are to evaluate whether POCUS provides and accurate alternative for differentation between normal- and haemorrhagic effusion. An overview of imaging modalities for haemophilic arthropathy with their potential indications is presented in Table 2.

Table 2. Imaging modalities for haemophilic arthropathy with their potential indications

\begin{tabular}{lll}
\hline X-rays & MRI & POC-US \\
\hline $\begin{array}{l}\text { First step for evaluation } \\
\text { of bony changes though }\end{array}$ & $\begin{array}{l}\text { Potentially useful for differentiation between normal- and } \\
\text { haemorrhagic effusion in patients }\end{array}$ & with painful joints as required \\
insensitive for initial changes & treatment differs & \\
+ Follow-up of arthropathy to & + Detection of synovial & Monitoring and guidance of \\
evaluate disease progression & hypertrophy, haemosiderin, and $/$ & treatment by evaluation of \\
(effect of treatment) & $\begin{array}{l}\text { or cartilage defects to evaluate } \\
\text { disease progression or to explain }\end{array}$ & $\begin{array}{l}\text { synovial hypertrophy as its } \\
\text { presence is associated with an }\end{array}$ \\
$+\begin{array}{l}\text { Surgical planning and follow- } \\
\text { up }\end{array}$ & the origin of clinical complaints & increased bleeding risk
\end{tabular}

In summary, current imaging modalities for haemophilic arthropathy include $\mathrm{X}$-rays, MRI, and POC-US depending on the articular changes of interest. Both MRI and POC-US allow for evaluation of synovial hypertrophy, though standardization of MRI and POC-US, as well as external validation of our findings is needed. 


\section{References}

1 Jansen NW, Roosendaal G, Lafeber FP. Understanding haemophilic arthropathy: an exploration of current open issues. Br J Haematol 2008; 143: 632-40.

2 Lafeber FPJG, Miossec P, Valentino L a. Physiopathology of haemophilic arthropathy. Haemophilia 2008; 14: 3-9.

3 Arnold WD, Hilgartner MW. Hemophilic arthropathy. Current concepts of pathogenesis and management. J Bone Jt Surg Am 1977; 59: 287-305.

4 Aronstam A, Rainsford SG, Painter MJ. Patterns of bleeding in adolescents with severe haemophilia A. Br Med J 1979; 1: 469-70.

5 Ahlberg A. Haemophilia in Sweden. VII. Incidence, treatment and prophylaxis of arthropathy and other musculo-skeletal manifestations of haemophilia A and B. Acta Orthop Scand Suppl 1965 Suppl $77: 3-132$

6 Fischer K, Steen Carlsson K, Petrini P, et al. Intermediate-dose versus high-dose prophylaxis for severe hemophilia: comparing outcome and costs since the 1970s. Blood 2013; 122: 1129-36.

7 den Uijl I, Biesma D, Grobbee D, Fischer K. Turning severe into moderate haemophilia by prophylaxis: are we reaching our goal? Blood Transfus 2013; 11: 364-9.

8 Löfqvist T, Nilsson IM, Berntorp E, Pettersson H. Haemophilia prophylaxis in young patients--a long-term follow-up. J Intern Med 1997; 241: 395-400.

9 Nijdam A, Foppen W, de Kleijn P, et al. Discontinuing early prophylaxis in severe haemophilia leads to deterioration of joint status despite low bleeding rates. Thromb Haemost 2016; doi:10.1160/ TH15-08-0637 [Epub ahead of print].

10 Pettersson H, Ahlberg A, Nilsson IM. A radiologic classification of hemophilic arthropathy. Clin Orthop Relat Res 1980; 149: 153-9.

11 Srivastava A, Brewer AK, Mauser-Bunschoten EP, et al. Guidelines for the management of hemophilia. Haemophilia 2013; 19: e1-47.

12 Fischer K, van der Bom JG, Molho P, et al. Prophylactic versus on-demand treatment strategies for severe haemophilia: a comparison of costs and long-term outcome. Haemophilia 2002; 8: 745-52.

13 Fischer K, Astermark J, van der Bom JG, et al. Prophylactic treatment for severe haemophilia: comparison of an intermediate-dose to a high-dose regimen. Haemophilia 2002; 8: 753-60.

14 Fischer K, van der Bom JG, Mauser-Bunschoten EP, et al. The effects of postponing prophylactic treatment on long-term outcome in patients with severe hemophilia. Blood 2002; 99: 2337-41.

15 Fischer K, Van Der Bom JG, Prejs R, et al. Discontinuation of prophylactic therapy in severe haemophilia: incidence and effects on outcome. Haemophilia 2001; 7: 544-50.

16 Foppen W, van der Schaaf IC, Beek FJ a., Verkooijen HM, Fischer K. Scoring haemophilic arthropathy on X-rays: improving inter- and intra-observer reliability and agreement using a consensus atlas. Eur Radiol 2015; doi: 10.1007/s00330-015 - 4013-8. [Epub ahead of print].

17 Willemink MJ, De Jong P a., Leiner T, et al. Iterative reconstruction techniques for computed tomography Part 1: Technical principles. Eur Radiol 2013; 23: 1623-31.

18 Willemink MJ, Leiner T, de Jong PA, et al. Iterative reconstruction techniques for computed tomography part 2: initial results in dose reduction and image quality. Eur Radiol 2013; 23: 1632-42. 
19 UC San Diego. Radiation Risk Statement Calculator 2015 Available at https://ehs.ucsd.edu/ Radiation_Risk/.

20 Manco-Johnson MJ. Prophylaxis versus episodic treatment to prevent joint disease in boys with severe hemophilia. N Engl J Med 2007; 2007: 535-44.

21 Chan MW, Leckie A, Xavier F, et al. A systematic review of MR imaging as a tool for evaluating haemophilic arthropathy in children. Haemophilia 2013; 19: e324-34.

22 Nuss R, Kilcoyne RF, Geraghty S, et al. MRI findings in haemophilic joints treated with radiosynoviorthesis with development of an MRI scale of joint damage. Haemophilia 2000; 6: 162-9.

23 Soler R, López-Fernández F, Rodríguez E, Marini M. Hemophilic arthropathy. A scoring system for magnetic resonance imaging. Eur Radiol 2002; 12: 836-43.

24 Lundin B, Pettersson H, Ljung R. A new magnetic resonance imaging scoring method for assessment of haemophilic arthropathy. Haemophilia 2004; 10: 383-9.

25 Lundin B, Babyn P, Doria a S, et al. Compatible scales for progressive and additive MRI assessments of haemophilic arthropathy. Haemophilia 2005; 11: 109-15.

26 Lundin B, Manco-Johnson ML, Ignas DM, et al. An MRI scale for assessment of haemophilic arthropathy from the International Prophylaxis Study Group. Haemophilia 2012; 18: 962-70.

27 Foppen W, Sluiter D, Witkamp TD, Mali WPTM, Fischer K. Haemophilic magnetic resonance imaging score in healthy controls playing sports. Haemophilia 2013; 19: 939-43.

28 Foppen W, van der Schaaf IC, Witkamp TD, Fischer K. Is joint effusion on MRI specific for haemophilia? Haemophilia 2014; 20: 582-6.

29 Foppen W, Fischer K. Prognostic Value of MRI Synovial Changes in Haemophilia Patients: Preliminary Results. Haemophilia 2015; 21 (s2): 41-2.

30 Timmer MA, Pisters MF, de Kleijn P, et al. Differentiating between signs of intra-articular joint bleeding and chronic arthropathy in haemophilia: a narrative review of the literature. Haemophilia 2015; 21: 289-96.

31 Nuss R, Kilcoyne R. The MRI Atlas of Hemophilic Arthropathy. NewYork: Professional Publishing Group, Ltd; 2002.

32 Hemke R, Kuijpers TW, Van Den Berg JM, et al. The diagnostic accuracy of unenhanced MRI in the assessment of joint abnormalities in juvenile idiopathic arthritis. Eur Radiol 2013; 23: 1998-2004.

33 Lundin B, Berntorp E, Pettersson H, et al. Gadolinium Contrast Agent is of Limited Value for Magnetic Resonance Imaging Assessment of Synovial Hypertrophy in Hemophiliacs. Acta Radiol 2007; 48: 520-30.

34 Di Minno MND, Iervolino S, Soscia E, et al. Magnetic resonance imaging and ultrasound evaluation of "healthy" joints in young subjects with severe haemophilia A. Haemophilia 2013; 19: e167-73.

35 Sierra Aisa C, Lucía Cuesta JF, Rubio Martínez A, et al. Comparison of ultrasound and magnetic resonance imaging for diagnosis and follow-up of joint lesions in patients with haemophilia. Haemophilia 2014; 20: e51-7.

36 Doria AS, Keshava SN, Mohanta A, et al. Diagnostic Accuracy of Ultrasound for Assessment of Hemophilic Arthropathy: MRI Correlation. Am J Roentgenol 2015; 204: W336-47.

37 Moore CL, Copel JA. Point-of-Care Ultrasonography. N Engl J Med 2011; 364: 749-57. 
38 Martinoli C, Della Casa Alberighi O, di Minno G, et al. Development and definition of a simplified scanning procedure and scoring method for Haemophilia Early Arthropathy Detection with Ultrasound (HEAD-US). Thromb Haemost 2013; 109: 1170-9.

39 Foppen W, van der Schaaf IC, Fischer K. Value of routine ultrasound in detecting early joint changes in children with haemophilia using the "Haemophilia Early Arthropathy Detection with UltraSound" protocol. Haemophilia 2016; 22: 121-5.

40 Acharya SS, Schloss R, Dyke JP, et al. Power Doppler sonography in the diagnosis of hemophilic synovitis-a promising tool. J Thromb Haemost 2008; 6: 2055-61.

41 Ceponis A, Wong-Sefidan I, Glass CS, von Drygalski A. Rapid musculoskeletal ultrasound for painful episodes in adult haemophilia patients. Haemophilia 2013; 19: 790-8.

42 Bhat V, Olmer M, Joshi S, et al. Vascular remodeling underlies rebleeding in hemophilic arthropathy. Am J Hematol 2015, doi: 10.1002/ajh.24133. [Epub ahead of print].

43 Strike KL, Iorio A, Jackson S, et al. Point of care ultrasonography in haemophilia care: recommendations for training and competency evaluation. Haemophilia 2015; 21: 828-31.

44 Fischer K, Oldenburg J, Astermark J, et al. Ultrasound evaluation of haemophilic joints by haemophilia physicians: a reliablity study. J Thromb Haemost 2015; 13 (s2): 601.

45 Nederlandse Zorgautoriteit. Tarieventabel DBC-zorgproducten en overige producten per 1 januari 20162015.

46 Zukotynski K, Jarrin J, Babyn PS, et al. Sonography for assessment of haemophilic arthropathy in children: a systematic protocol. Haemophilia 2007; 13: 293-304.

47 Keshava SN, Gibikote S, Mohanta A, Doria a. S. Refinement of a sonographic protocol for assessment of haemophilic arthropathy. Haemophilia 2009; 15: 1168-71.

48 Melchiorre D, Linari S, Innocenti M, et al. Ultrasound detects joint damage and bleeding in haemophilic arthropathy: a proposal of a score. Haemophilia 2011; 17: 112-7.

49 Querol F, Rodriguez-Merchan EC. The role of ultrasonography in the diagnosis of the musculoskeletal problems of haemophilia. Haemophilia 2012; 18: e215-26.

50 Doria A, Lundin B, Kilcoyne R, et al. Reliability of progressive and additive MRI scoring systems for evaluation of haemophilic arthropathy in children: expert MRI Working Group of the International Prophylaxis Study Group. Haemophilia 2005; 11: 245-53. 
Chapter 9

Summary 
Repeated provoked or spontaneous bleeding into the joints are the hallmark of severe haemophilia. Recurrent or prolonged joint bleeds eventually lead to synovial hypertrophy, progressive cartilage degradation and bone damage through mechanical and metabolic joint destruction. The effects of previous joint bleeding and clotting factor replacement therapy can be assessed using imaging of joints in haemophilia patients. In addition, imaging of haemophilic arthropathy allows comparison of different clotting factor replacement strategies.

\section{Part I: X-rays}

As standard X-rays are inexpensive and universally available, the radiological Pettersson score is widely applied for classification of haemophilic arthropathy. The inter- and intra-observer reliability and agreement (reproducibility) of the Pettersson score was assessed and improved using a newly developed consensus atlas in chapter 2. X-rays of in total 240 joints of 20 patients including the whole range of haemophilic arthropathy were scored (Pettersson score at joint level (PS joint $_{\text {) }}$ 0-13 points). A consensus atlas with example images and descriptions of the items was made and is available online [booklet: goo.gl/TYQyLb, interactive format: goo.gl/ohsVaM]. Using the consensus atlas, inter-observer reliability of the $\mathrm{PS}_{\text {ioint }}$ improved significantly from 0.94 (95\% confidence interval (CI) $0.91-0.96)$ to 0.97 (CI 0.96-0.98). Limits of agreement improved from \pm 1.7 to \pm 1.1 from the mean PS ${ }_{\text {joint }}$. Intra-observer reliability of the PS $_{\text {joint }}$ was 0.98 (CI 0.97-0.98), and intra-observer limits of agreement were \pm 0.9 points. True differences in arthropathy can be interpreted as differences in the $\mathrm{PS}_{\text {joint }}$ of $>2$ points. In conclusion, use of a consensus atlas improved reliability and agreement of the Pettersson score.

\section{Part II: Magnetic Resonance Imaging}

From a clinical perspective, it is important to assess early, potentially reversible, joint changes in patients with normal findings on physical examination and X-rays. Currently there is an increasing interest in the use of Magnetic Resonance Imaging (MRI) as it is the most sensitive imaging modality to assess joint lesions.

A cohort of young active healthy men was evaluated by MRI in chapter 3 in order to provide context for joint evaluation by MRI in young haemophilic 
patients. Both knees and ankles of 30 healthy men aged 18-26 years, regularly active in sports, were scanned using 3 Tesla MRI. Clinical function was assessed on the same day using the Haemophilia Joint Health Score. Although 7\% of joints showed abnormalities on MRI, no haemophilia specific joint changes were found and clinical function was optimal in $88 \%$ of joints. To conclude, regular sports participation or very low Haemophilia Joint Health Scores are not associated with haemophilia specific MRI changes in knees and ankles. Therefore, it is unlikely that MRI changes in young active haemophilia patients are caused by sports participation only.

In chapter 4 the specificity of joint effusion in haemophilia patients was evaluated by comparison with joint effusion in healthy controls. In total 104 joint (52 knees, 52 ankles) of 26 haemophilia patients and 120 joints (60 knees, 60 ankles) of 30 healthy young men (controls) were assessed. Joint effusion according to criteria of the IPSG MRI scale was significantly more prevalent in knees of healthy controls, while findings in ankles were similar. Using multivariate regression, a significant positive association was found between effusion and frequency of sports for knees only. Age and MRI score were not associated with the amount of knee effusion. Ankle joints, in both patients and controls, showed minimal effusion only and was not associated with age, frequency of impact sports, or MRI score. These data suggest that joint effusion in knees and ankles is not haemophilia specific. Inclusion of joint effusion in the IPSG MRI scale is expected to reduce its specificity for haemophilic arthropathy.

The predictive value of MRI findings for joint bleeding and progression of arthropathy was assessed in chapter 5. In total, 104 joints of 26 haemophilia patients (aged 12-29 years) with absent or minimal arthropathy on X-rays were scanned with MRI. Five years later, joint bleeding was reported for 36\% of joints in total. Joints with synovial hypertrophy on MRI bled earlier $(P<0.01)$ and more often $(P<0.01)$ compared to joint without synovial hypertrophy. The absolute risk of any five year bleeding was $80 \%$ for joints with synovial hypertrophy compared to $27 \%$ in joints without synovial hypertrophy (adjusted OR: 9.1, $P<0.05)$. Any MRI finding according to the IPSG MRI score, except joint effusion, was predictive for progression of arthropathy. For apparently healthy joints on X-rays, the positive predictive value of MRI findings for 5 -year X-ray changes was 75\% and the negative predictive value was $98 \%$. MRI evaluation provides relevant information as observed changes were associated with an increased bleeding risk and progression of arthropathy. 


\section{Part III: Point-of-care ultrasound}

Irrespective of the benefits of MRI over standard X-rays, MRI is not the first choice for routine joint assessment in the absence of major clinical complaints. Ultrasound may be an alternative for MRI as it can visualize fluid collections, synovial hypertrophy in the joint recesses, and parts of articular surfaces. A recently developed point-of-care ultrasound (POC-US) protocol for non-radiologists was evaluated in this this.

The objective of chapter 6 was to investigate whether POC-US may be an accurate alternative for MRI for joint evaluation in haemophilia patients. Bilateral knees and ankles of 24 haemophilia patients with limited arthropathy (96 joints), aged 18-31 years, were scanned with ultrasound and 3 Tesla MRI. POC-US for synovial tissue was correct in 97\% (CI: 91-99) of joints with a positive predictive value of 94\% (CI: 73-100) and a negative predictive value of 97\% (CI: 91-100). The accuracy of POC-US was 91\% (CI: 83-96) for cartilage defects and 97\% (CI: 91-99) for bone defects. In conclusion, POCUS could accurately assess synovial hypertrophy in haemophilia patients with limited joint disease. As synovial hypertrophy is associated with bleeding, POC-US performed by trained clinicians may help to tailor haemophilia treatment.

In chapter 7 the value POC-US in detecting early joint abnormalities was explored in children with haemophilia. 64 joints in 32 children (aged 6-16 years) with haemophilia were examined by one operator using a POC-US protocol during annual multidisciplinary follow-up. Based on reported bleeding, the joint with the highest risk of blood-induced joint damage and the contralateral joint were chosen for ultrasound evaluation. Clinical function was assessed according to the Haemophilia Joint Health Score. POC-US showed abnormalities in 10\% of joints with reported bleeding. POC-US abnormalities were present in $80 \%$ of joints with limited loss of clinical function and in $2 \%$ of apparently normal joints according to physical examination. Therefore, POC-US may be used in children to evaluate whether minimal losses of clinical function might be caused by anatomical changes. 


\section{Addenda}

Nederlandse samenvatting (Dutch summary) Review committee Dankwoord (Acknowledgements) Biography List of publications 


\section{Beeldvorming van gewrichtsschade ten gevolge van hemofilie}

Patiënten met hemofilie hebben regelmatig gewrichtsbloedingen als gevolg van een tekort aan stollingsfactoren in het bloed. Herhaalde of langdurige gewrichtsbloedingen leiden uiteindelijk tot zwelling van de binnenbekleding van het gewricht (synoviale zwelling) en toenemende gewrichtsschade. De gevolgen van deze gewrichtsbloedingen kunnen onder andere beoordeeld worden middels röntgenfoto’s, MRI en echografie.

Röntgenfoto's worden veelvuldig gebruikt om de mate van gewrichtsschade te bepalen. Hiervoor is door Pettersson een veelgebruikte scoringsmethode ontwikkeld. In dit proefschrift werd de reproduceerbaarheid van die scoringsmethode onderzocht en verbeterd door gebruik te maken van een nieuw ontwikkelde atlas met voorbeelden van afwijkingen.

MRI wordt steeds vaker gebruikt om vroege gewrichtsschade te detecteren. De betekenis van de bevindingen op MRI-scans is echter niet altijd duidelijk. Om MRI-scans bij patiënten met hemofilie te interpreteren werd in dit proefschrift eenmalig een groep jonge mannen zonder hemofilie onderzocht. Ook werd een groep jonge mannen met hemofilie vijf jaar lang gevolgd. De resultaten suggereren onder andere dat vocht in het gewricht niet specifiek is voor hemofilie. Verder bleek in de patiëntengroep dat gewrichten met zwelling van de binnenbekleding van het gewricht een verhoogd risico hebben op bloedingen en gewrichtsschade na vijf jaar.

Echografie is beter beschikbaar en goedkoper dan MRI. Dit proefschrift laat zien dat echografie een nauwkeurig alternatief is voor MRI om de aanof afwezigheid van zwelling van de binnenbekleding van het gewricht te onderzoeken. Mogelijk kunnen de bevindingen in dit proefschrift worden gebruikt om de behandeling van hemofilie te optimaliseren met behulp van beeldvorming. 


\section{Addenda}

Nederlandse samenvatting (Dutch summary)

Review committee

Dankwoord (Acknowledgements)

Biography

List of publications 
Prof. dr. T. Leiner

Professor of Cardiovascular Radiology

Department of Radiology, University Medical Center Utrecht

Prof. dr. D.B.F. Saris

Professor of Reconstructive Medicine

Department of Orthopaedics, University Medical Center Utrecht; and Faculty of Science and Technology, University of Twente

Prof. dr. F.P.J.G. Lafeber

Professor of Experimental Rheumatology

Department of Rheumatology \& Clinical Immunology, University Medical Center Utrecht

Prof. dr. J. Hendrikse

Professor of Neuroradiology

Department of Radiology, University Medical Center Utrecht

Prof. dr. M. Maas

Professor of Musculoskeletal Radiology

Department of Radiology, Academic Medical Center, Amsterdam 


\section{Addenda}

\section{Nederlandse samenvatting (Dutch summary) Review committee}

Dankwoord (Acknowledgements) Biography List of publications 


\section{Addenda}

Nederlandse samenvatting (Dutch summary)

Review committee

Dankwoord (Acknowledgements)

Biography

List of publications 


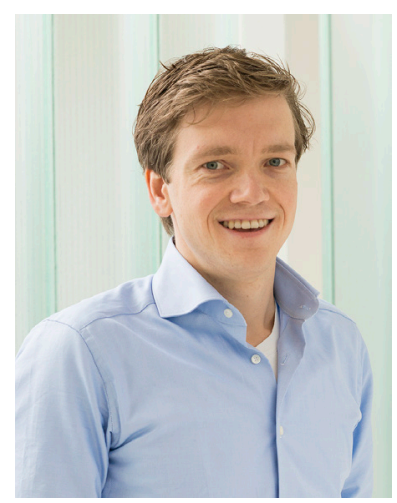

Wouter Foppen was born on the $24^{\text {th }}$ of January 1988 in Harderwijk, the Netherlands. After completing high school in 2006 (RSG Slingerbos in Harderwijk) he moved to Utrecht to start medical school at the Utrecht University in the same year. After his senior internships in Radiology at the Meander Medical Center in Amersfoort he started with a research project at the University Medical Center (UMC) Utrecht. This collaborative research project between the Van Creveldkliniek (Department of Haematology) and Department of Radiology was performed under supervision of dr. K. Fischer and prof. dr. W.P.T.M. Mali. After graduating from medical school in 2012 he continued the research project on Imaging of Haemophilic Arthropathy as a PhD-student, under supervision of the same supervisors and dr. I.C. van der Schaaf. The results of this collaborative research project are presented in this thesis. During his $\mathrm{PhD}$ he completed a Postgraduate Master in Clinical Epidemiology at the Utrecht University in 2015. In December 2015 he started his Radiology training at the UMC Utrecht under supervision of dr. R.A.J. Nievelstein. 


\section{Addenda}

Nederlandse samenvatting (Dutch summary)

Review committee

Dankwoord (Acknowledgements)

Biography

List of publications 


\section{Peer-reviewed publications}

Nijdam A, Foppen W, De Kleijn P, Mauser-Bunschoten EP, Roosendaal G, van Galen KP, Schutgens RE, van der Schouw Y'T, Fischer K. Discontinuing early prophylaxis in severe haemophilia leads to deterioration of joint status despite low bleeding rates. Thromb Haemost. 2016. [Epub ahead of print]

Willemink MJ, den Harder AM, Foppen W, Schilham AM, Rienks R, Laufer EM, Nieman K, de Jong PA, Budde RP, Nathoe HM, Leiner T. Finding the optimal dose reduction and iterative reconstruction level for coronary calcium scoring. J Cardiovasc Comput Tomogr. 2016;10(1):69-75.

Foppen W, van der Schaaf IC, Fischer K. Value of routine ultrasound in detecting early joint changes in children with haemophilia using the 'Haemophilia Early Arthropathy Detection with UltraSound' protocol. Haemophilia. 2016;22(1):121-5.

Foppen W, van der Schaaf IC, Beek FJ, Verkooijen HM, Fischer K. Scoring haemophilic arthropathy on X-rays: improving inter- and intra-observer reliability and agreement using a consensus atlas. Eur Radiol. 2015. [Epub ahead of print]

Foppen W, van der Schaaf IC, Witkamp TD, Fischer K. Is joint effusion on MRI specific for haemophilia? Haemophilia. 2014;20(4):582-6.

Sluiter D, Foppen W, de Kleijn P, Fischer K. Haemophilia Joint Health Score in healthy adults playing sports. Haemophilia. 2014;20(2):282-6.

Foppen W, Sluiter D, Witkamp TD, Mali WP, Fischer K. Haemophilic magnetic resonance imaging score in healthy controls playing sports. Haemophilia. 2013;19(6):939-43. 


\section{Conference presentations}

Timmer MA, Foppen W, Schutgens REG, Pisters MPF, Fischer K. Comparing findings of physical examination and ultrasound in adults with haemophilia: a pilot study. 9th Congress of the European Association for Haemophilia and Allied Disorders, Malmö, 2016 (poster presentation).

Nijdam A, Foppen W, de Kleijn P, Mauser-Bunschoten E, Roosendaal G, van Galen K, Schutgens R, Fischer K. Discontinuing early prophylaxis in young adults with severe haemophilia A: Deterioration of joint status after 10 years despite low bleeding rates. International Society on Thrombosis and Haemostasis 2015 Congress, Toronto, 2015 (oral presentation).

Foppen W, van der Schaaf IC, Fischer K. Predicting hemarthroses and progression of arthropathy in hemophilia patients: Preliminary results on the prognostic value of MRI synovial changes. 14th World Federation of Hemophilia International Musculoskeletal Congress, Belfast, 2015 (oral presentation).

den Harder AM, Willemink MJ, Foppen w, Schilham AMR, Budde RPJ, Leiner T, de Jong PA. Achievable dose reduction for coronary calcium scoring with iterative reconstruction. European Congress of Radiology, Vienna, 2015 (poster presentation).

Foppen W, van der Schaaf IC, Beek FJA, Verkooijen HM, Fischer K. Scoring haemophilic arthropathy on $\mathrm{X}$-rays: Improving reproducibility of the Pettersson score using a consensus atlas. 8th Congress of the European Association for Haemophilia and Allied Disorders, Helsinki, 2015 (poster presentation).

Foppen W, van der Schaaf IC, Fischer K. Prognostic value of MRI synovial changes in haemophilia patients: preliminary results. 8th Congress of the European Association for Haemophilia and Allied Disorders, Helsinki, 2015 (poster presentation).

Willemink MJ, den Harder AM, Foppen W, Nathoe HM, de Jong PA, Budde RPJ, Leiner T. Finding the Optimal Dose Reduction and Iterative Reconstruction Level for Coronary Calcium Scoring: A Within Patients Analysis. Radiological Society of North America Annual Meeting, Chicago, 2014 (poster presentation). 
Foppen W, van der Schaaf IC, Beek FJA, Fischer K. Improving inter-observer variability of the Pettersson score using a consensus atlas. World Federation of Hemophilia Congress, Melbourne, 2014 (poster presentation).

Foppen W, van der Schaaf IC, Fischer K. Ultrasound screening for early arthropathy in children with moderate and severe haemophilia: first results. 7th Congress of the European Association for Haemophilia and Allied Disorders, Brussels, 2014 (oral presentation).

Foppen W, Sluiter D, Witkamp TD, Mali WPTM, Fischer K. Haemophilic MRI score in healthy controls playing sports. 6th Congress of the European Association for Haemophilia and Allied Disorders, Warsaw, 2013 (poster presentation). 
